NBER WORKING PAPER SERIES

\title{
FROM FOG TO SMOG: THE VALUE OF POLLUTION INFORMATION
}

\author{
Panle Jia Barwick \\ Shanjun Li \\ Liguo Lin \\ Eric Zou \\ Working Paper 26541 \\ http://www.nber.org/papers/w26541 \\ NATIONAL BUREAU OF ECONOMIC RESEARCH \\ 1050 Massachusetts Avenue \\ Cambridge, MA 02138 \\ December 2019, Revised January 2023
}

\begin{abstract}
We thank the editor and three anonymous referees whose detailed comments have greatly improved the paper. We also thank Doug Almond, Antonio Bento, Fiona Burlig, Trudy Cameron, Lucas Davis, Todd Gerarden, Jiming Hao, Guojun He, Zhiguo He, Joshua Graff Zivin, Matt Khan, Jessica Leight, Cynthia Lin Lowell, Grant McDermott, Francesca Molinari, Ed Rubin, Ivan Rudik, Joe Shapiro, Jeff Shrader, Jörg Stoye, Jeffrey Zabel, Shuang Zhang, and seminar participants at the 2019 NBER Chinese Economy Working Group Meeting, the 2019 NBER EEE Spring Meeting, the 2019 Northeast Workshop on Energy Policy and Environmental Economics, MIT, Resources for the Future, University of Alberta, University of Chicago, Cornell University, GRIPS Japan, Indiana University, Shanghai University of Finance and Economics, University of Kentucky, University of Maryland, University of Oregon, University of Texas at Austin, and Xiamen University for helpful comments. We thank Jing Wu and Ziye Zhang for their generous help with data, and Luming Chen, Deyu Rao, Binglin Wang, and Tianli Xia for outstanding research assistance. Barwick gratefully acknowledges the generous support by the National University of Singapore during her sabbatical visit. The views expressed herein are those of the authors and do not necessarily reflect the views of the National Bureau of Economic Research.
\end{abstract}

NBER working papers are circulated for discussion and comment purposes. They have not been peer-reviewed or been subject to the review by the NBER Board of Directors that accompanies official NBER publications.

(C) 2019 by Panle Jia Barwick, Shanjun Li, Liguo Lin, and Eric Zou. All rights reserved. Short sections of text, not to exceed two paragraphs, may be quoted without explicit permission provided that full credit, including $\odot$ notice, is given to the source. 
From Fog to Smog: the Value of Pollution Information

Panle Jia Barwick, Shanjun Li, Liguo Lin, and Eric Zou

NBER Working Paper No. 26541

December 2019, Revised January 2023

JEL No. D80,I10,Q53,Q58

\section{$\underline{\text { ABSTRACT }}$}

In 2013, China launched a landmark program to monitor air quality and disclose real-time data, significantly increasing the public's access to and awareness of pollution information. The program triggered cascading behavioral changes such as stronger avoidance of outdoor pollution exposure and increased spending on protective products. These behavioral responses mitigated the mortality impact of air pollution. Conservative estimates indicate that the program's health benefits outweigh the costs by an order of magnitude. The findings highlight the benefits of improving public access to pollution information in developing countries which often experience severe air pollution but lack pollution data collection and dissemination.

Panle Jia Barwick

University of Wisconsin-Madison

Department of Economics

1180 Observatory Dr

Madison, WI 53706

and NBER

panle.barwick@gmail.com

Shanjun Li

Cornell University

405 Warren Hall

Ithaca, NY 14853

and NBER

SL2448@ cornell.edu
Liguo Lin

Institute for Advanced Research

Shanghai University of Finance and Economics

Shanghai

China

lin.liguo@mail.shufe.edu.cn

Eric Zou

Department of Economics

University of Oregon

1415 Kincaid Street

Eugene, OR 97403

and NBER

ericzou@uoregon.edu 


\section{Introduction}

While many developing countries are experiencing some of the worst pollution in the world, residents in these countries lack access to credible and readily available pollution information. This information is either not collected, deliberately kept secret, or intentionally distorted by their governments. ${ }^{1}$ Although economists have long emphasized the importance of information in decision-making (Stigler, 1961), little is known about the effects of collecting and disseminating pollution information on the daily lives of citizens in developing countries. Filling this knowledge gap is important, particularly because public funding for improving information infrastructure in developing countries competes with meeting basic needs in health care, nutrition, and education.

China provides a perfect setting for studying the value of pollution information, both because of the high levels of air pollution it has experienced and because of the sharp and sudden change of its policy to monitor and disclose real-time air quality information. During the 2000s, daily average concentrations of fine particulate matter $\left(\mathrm{PM}_{2.5}\right)$ in China is estimated to exceed $50 \mathrm{ug} / \mathrm{m}^{3}$, five times above the World Health Organization guideline. Despite the hazardous pollution levels, a comprehensive monitoring network did not exist. Dissemination of the scant data that were collected was politically controlled and, in many cases, forbidden. As a result, there was widespread confusion and obfuscation among government agencies and the general public about whether fog or severe air pollution caused murky skies. $^{2}$ In 2013, amid social outcry over the lack of transparency, China made a dramatic change: it launched a nationwide program that began monitoring air quality and disclosing the information to the public in real time (henceforth, the monitoring program). This landmark change represents an important moment in the history and evolution of China's environmental regulations, and the implementation of the program offers a unique opportunity to study how the availability of real-time information on air pollution levels affect household behavior and individual health.

We present the first empirical analysis of this natural experiment and our analysis proceeds in two steps. First, we document what happens to pollution information availability and citizen awareness once public air quality information became widely available. Second,

\footnotetext{
${ }^{1}$ In 2018, among the 20 countries with the worst fine particulate matter $\left(\mathrm{PM}_{2.5}\right)$ pollution (with annual median concentration $>46 \mathrm{ug} / \mathrm{m}^{3}$ ), only four (Nepal, Saudi Arabia, India, and China) have installed a pollution monitoring system.

${ }^{2}$ Both fog and smog could cause low visibility and mostly occur in the fall and winter months, contributing to confusion among the general public. However, fog and smog have different compositions with dramatically different health implications. Fog forms when water vapors under humid conditions condense into water droplets or ice crystals that are suspended in the air near the ground. Smog, a type of intense air pollution, is caused by aerosol particles such as $\mathrm{PM}_{2.5}$ that carry toxins and hence impair health when breathed in.
} 
we study how the monitoring program affects behavioral and health outcomes, focusing on two central objects of the literature: avoidance behavior and the health effects of air pollution. We measure these objects by examining how outdoor activities and health outcomes respond to short-term pollution fluctuations, which we will henceforth refer to as the pollution "gradients", $\frac{\partial \text { Outdoor Activities }}{\partial \text { Pollution }}$ and $\frac{\partial \text { Health }}{\partial \text { Pollution }}$. Our empirical focus is to estimate how the monitoring program changed these gradients. The basis of our identification strategy is the staggered introduction of the monitoring program across three waves of cities and completed over the course of two years. The sequence of the staggered rollout was a top-down decision based on administrative hierarchies and characteristics determined long before the program. ${ }^{3}$ Thus, the rollout schedule was uncorrelated with the day-to-day variation of local pollution levels, as validated in our empirical analysis. In addition, there were no other programs or regulations following a similar rollout schedule during our sample period. Importantly, our identification strategy uses an econometric insight - in particular, two orthogonality conditions that can be tested directly with data - which allows us to causally identify the changes in the two gradients using OLS regressions.

We build the most comprehensive database yet compiled on a rich set of outcomes including social awareness of air pollution, air quality measures from satellites, economic activities, and health outcomes - that covers the period before and after the monitoring program. We first document that the monitoring program has profoundly transformed the landscape of public access to pollution information and dramatically increased households' awareness about pollution issues. The frequency of the publication of air pollution-related articles in People's Daily, the government's official newspaper, rose from less than once per week to almost daily. Immediately after the program was launched, the term "smog" ("wu mai" in Chinese) became, for the first time, a buzzword in social media. National surveys indicate that respondents' concern about environmental issues has significantly increased following the program, with the increase in concern being proportionate to local air pollution levels in where the respondent resides.

The changes in information access and in public awareness triggered a cascade of behavioral changes. Our analysis takes advantage of the universe of credit card and debit card transactions that occurred at physical stores in China from 2011 to 2016. We measure the extent to which people avoid pollution by analyzing the "shopping-pollution gradient", which reflects how outdoor shopping trips vary with weekly fluctuations in ambient pollution, and evaluate how the monitoring program impacts this gradient. We show that the monitoring

\footnotetext{
${ }^{3}$ This includes characteristics such as the location of the city (e.g. whether it is located in a special economic zone), its status as a provincial capital, and any prior designations it may have received (e.g. being designated as an environmental exemplary city in 2007).
} 
program boosted pollution avoidance by triggering a negative shopping-pollution elasticity of 1.4 percent, a pattern that is robust across a host of econometric specifications. The increase in avoidance concentrates in plausibly "deferrable" consumption categories, including supermarket shopping, restaurant dining, and entertainment. We further examine the impact of the monitoring program on defensive purchases using a separate dataset on monthly air purifier sales in 50 large cities in China. We find that the monitoring program substantially intensified defensive spending: within one year of the program implementation in a city, purchases of air purifiers almost doubled and became highly correlated with contemporaneous local pollution levels.

The evidence indicates that there is a strong demand for public information on air pollution and that households gained new information from the government monitoring program based on the observed changes in behavior. It is natural to ask whether households obtained their own (proxy) information about air pollution in the absence of government-provided monitoring data before the monitoring program. We consider two potential sources of private information. First, we examine whether people can use visibility to infer pollution levels. We show that the relationship between visibility and pollution is noisy, especially when visibility is poor which could arise from either fog or smog. Second, we consider the city of Beijing where the U.S. Embassy has conducted independent pollution monitoring since 2008. The Embassy data were released on Twitter, which was (and still is) banned in China, so most people do not have easy access to it. Our econometric analyses show that households were not able to effectively engage in pollution avoidance with these private sources of information prior to the monitoring program.

The behavioral changes we document hold the potential to mitigate some of the devastating health consequences of severe air pollution in China. In the final part of the paper, we examine changes in the mortality-pollution relationship that occurred over time as the public's access to information improved. Using nationally representative mortality data from the Chinese Center for Disease Control and Prevention (CDC), we find a 1.3-2.3 percentagepoint reduction in the mortality-pollution elasticity after monitoring began. The effect was concentrated among individuals aged 60 and above, and was more pronounced for deaths due to cardiovascular and respiratory causes than for other causes. As expected, the monitoring program had no effect on injury-related mortality.

Using estimates of the age-adjusted value of statistical life (VSL) and healthcare costs of air pollution in the literature (Ashenfelter and Greenstone, 2004; Murphy and Topel, 2006; Barwick et al., 2020), we calculate that risk-compensating behavioral responses to the monitoring program generated annual mortality and morbidity benefits of at least $¥ 93$ billion. Such social benefits outweigh the costs from defensive investments (such as air purifier 
purchases), avoidance behaviors (such as foregone consumption), and program deployment and maintenance by nearly one order of magnitude, making the monitoring program among the most successful environmental policies documented in a developing country.

We make three main contributions to the literature. First, to the best of our knowledge, our study provides the first empirical estimate of the costs and benefits of a nationwide program on pollution monitoring and information disclosure. In a concurrent study, Greenstone et al. (2022) documented that the automatic data collection underlying this national program improves the data quality and increases online searches for face masks and air filters. Our study complements theirs by showing that the program triggered a cascade of behavioral responses from increased awareness to pollution avoidance, which in turn reduced the health harms of air pollution. Our study is also closely related to the pioneering study by Neidell (2009) on the impact of pollution information on avoidance behavior. In a context where pollution information is widely available via both print and electronic media, the study shows that smog alerts in Southern California reduce attendance in two major outdoor facilities and that the resulting avoidance behavior mitigates the health impact of pollution. ${ }^{4}$ By examining a large-scale information program in China, our empirical findings highlight the considerable benefits of collecting and disseminating pollution information in developing countries, many of which are experiencing the world's worst mortality damage from pollution exposure (Landrigan et al., 2018). The success of China's program provides a benchmark for policy discussions (e.g., cost-benefit analysis) on building information infrastructure in these countries.

Second, our study shows that pollution information is a crucial determinant of avoidance behavior and defensive spending, consistent with findings in Neidell (2009) and Graff Zivin and Neidell (2009). Consumer activities (online searches, day-to-day shopping, and air purifier purchases) exhibit an insignificant response to pollution until such information becomes widely available. This suggests the importance of understanding the degree of consumers' access to information when applying the revealed preference method to estimate the value of non-market environmental goods (Gao, Song and Timmins, 2021). To the extent that access to information is lacking in developing countries, consumers' true willingness to pay for environmental goods could be underestimated. Our findings provide a potential explanation for why environmental quality is severely undervalued in developing countries (Greenstone and Jack, 2015), and why the dose-response relationship between pollution and mortality differs across developed and developing economies (Arceo, Hanna and Oliva, 2015).

\footnotetext{
${ }^{4}$ In the same context, a follow-up study by Graff Zivin and Neidell (2009) highlights that behaviors to avoid pollution risks (e.g., through intertemporal substitution) entails costs. A similar literature quantifies the value of weather forecasts, another type of government-provided information, as an important input to production decisions (Lave, 1963; Craft, 1998; Shrader, 2018; Jagnani et al., 2018).
} 
Third, this study contributes to the broad empirical literature on the role of information in consumer choices. Growing evidence suggests that consumers misperceive product attributes in a wide range of contexts. Examples include food nutritional contents (Bollinger, Leslie and Sorensen, 2011), insurance policy costs (Kling et al., 2012), vehicle fuel economies (Allcott, 2013), retirement savings (Bernheim, Fradkin and Popov, 2015), taxation levels (Chetty, Looney and Kroft, 2009), and energy prices (Shin, 1985; Ito, 2014). Information provision programs can improve consumers' perception of product attributes (Smith and Johnson, 1988; Oberholzer-Gee and Mitsunari, 2006), change consumer choices (Duflo and Saez, 2003; Hastings and Weinstein, 2008; Dranove and Jin, 2010; Jessoe and Rapson, 2014; Newell and Siikamäki, 2014; Mastromonaco, 2015; Wichman, 2017), and drive up average product quality (Jin and Leslie, 2003; Bai, 2018). In the context of air quality, recent studies have documented behavioral responses to pollution exposure over both the short and long terms. Our analysis shows that these behavioral responses could lead to significantly improved health outcomes at a moderate cost. We use the associated savings in monetary terms to provide a lower-bound estimate of the benefit of pollution information.

The rest of this paper is organized as follows: Section 2 reviews institutional details on the monitoring program and describes data sources. Section 3 documents the dramatic changes in information access and awareness after the program. Section 4 describes the empirical framework. Sections 5 and 6 present the effect of the program on avoidance behavior and on mortality. Section 7 calculates the costs and benefits of monitoring program. Section 8 concludes.

\section{Institutional Background and Data}

\subsection{Environmental Regulations}

The real-time $\mathrm{PM}_{2.5}$ monitoring program that started in 2013 was an important milestone in the history of China's environmental regulations. The program brought about a sharp and sudden change in the access of pollution information and drastically enhanced the public awareness of the health impact of $\mathrm{PM}_{2.5}$. This section aims to help understand the significance of and underlying factors behind the policy change.

A Brief Chronology of Environmental Regulations since 1982 In 1982, China established its first national ambient air quality standards (NAAQS), which set limits for multiple air pollutants including total suspended particulates (TSP), sulfur dioxide $\left(\mathrm{SO}_{2}\right)$, nitrogen oxides $\left(\mathrm{NO}_{2}\right)$, carbon monoxide (CO), and ozone $\left(\mathrm{O}_{3}\right)$ (Jiang, Li and Yang, 2020). 
A 1996 amendment expanded the standards to further include coarse particulate matter $\left(\mathrm{PM}_{10}\right)$. Throughout much of the 1980s, 1990s, and the early 2000s, the primary threat to air quality was considered to be $\mathrm{SO}_{2}$ due to coal burning. As acid rain caused widespread and visible damage to crops, forests, and the aquatic environment, central environmental regulations focused on the control of acid rain and $\mathrm{SO}_{2}$ emissions (Yi, Hao and Tang, 2007). The most prominent regulation was the two-control zone policy (TCZ) implemented in 1998 where cities with low $\mathrm{pH}$ values of precipitation and high $\mathrm{SO}_{2}$ concentration were designated as either the acid rain control zone located in southern China, or the $\mathrm{SO}_{2}$ control zone in northern China. Cities in these control zones were subject to a series of regulatory measures, such as mandatory installation of flue gas desulfurization in coal-fired power plants and closure of small coal-fired power plants (Tanaka, 2015). These aggressive policies reduced the average $\mathrm{SO}_{2}$ concentration by nearly $45 \%$ from 1990 to 2002, with the majority of cities achieving the national standard by 1998 (Hao and Wang, 2005).

Starting in early 2000, the source of air pollution changed from mainly coal burning to a mix of sources, due to declining emissions from coal-fired power plants and skyrocketing emissions from automobiles, manufacturing facilities, and construction. During this period, pollution regulation followed a federalism approach, where the central government established environmental standards that were meant to be met by local government efforts (Ghanem and Zhang, 2014). However, this approach proved to be ineffective due to the strong incentives for economic growth at the local level conflicting with weak monitoring and enforcement by the central government - a common issue in developing countries (Greenstone and Hanna, 2014; Duflo et al., 2018; Karplus, Zhang and Almond, 2018). Extreme episodes of air pollution were common in many urban areas, particularly during the winter.

In 2012, China's Ministry of Environmental Protection (MEP) revised the NAAQS and, for the first time in history, set the national standards for $\mathrm{PM}_{2.5}$. The new standards were slated to take effect nationwide in 2016. To help achieve the standards, China's State Council released the Action Plan on Air Pollution Prevention and Control in September 2013, which set specific targets for $\mathrm{PM}_{2.5}$ reduction from 2013 to 2017 and outlined concrete policies such as promoting the role of market-based mechanisms. It also established a monitoring and warning system to cope with severe air pollution events, which is the monitoring program analyzed in this paper. ${ }^{5}$ In March 2014, Premier Li Keqiang described smog as "nature's red-light warning against inefficient and blind development" and declared a "war on smog" at the opening of the annual meeting of People's Congress (Greenstone et al., 2021). For the

\footnotetext{
${ }^{5}$ The plan may have been China's most influential environmental policy during the past decade. Under this plan, $\mathrm{PM}_{2.5}$ fell by over $37 \%$ in the Beijing-Tianjin-Hebei Region, $35 \%$ in the Yangtze River Delta, $26 \%$ in the Pearl River Delta, and over 30\% on average in more than 70 major cities (Huang et al., 2018).
} 
first time in the history of national five-year plans, the 13th Five-Year Plan required cities at the prefecture level or higher to reduce $\mathrm{PM}_{2.5}$ concentrations by $18 \%$ from 2015 to 2020 (Wang, 2017).

China's nationwide pollution monitoring and disclosure program, as well as its aggressive policies to reduce $\mathrm{PM}_{2.5}$ concentrations marked a significant shift from the country's previous approach of prioritizing economic growth over environmental considerations. Several players and factors likely have worked together in contributing to the dramatic shift. These include a growing body of scientific knowledge on the pernicious effect of $\mathrm{PM}_{2.5}$ on human health, independent $\mathrm{PM}_{2.5}$ monitoring conducted by the U.S. Embassy in Beijing which have caused social media storms, and the increasing impacts of environmental NGOs and social media influencers. Other economic factors such as the rapid growth in household income, the emergence of a large middle class, and the corresponding increase in demand for environmental quality, may also have contributed to the shift (Kahn and Zheng, 2016). For interested readers, we provide detailed discussions of the policy shift and underlying factors in Appendix A, which presents a chronology of the key events from June 2004 when "smog" was first known to be used to describe the atmospheric condition to March 2014 when the Chinese Premier Li Keqiang declared the "war on smog".

Pollution Information Access and Public Awareness Before 2013 While air pollution has been a long-standing issue, public access to real-time pollution information, especially $\mathrm{PM}_{2.5}$ concentration, was nearly absent prior to 2013. The MEP began publishing a daily Air Pollution Index (API) based on reported data on $\mathrm{PM}_{10}, \mathrm{SO}_{2}$, and $\mathrm{NO}_{2}$ from local governments for over 40 major cities in 2000. ${ }^{6}$ There are several issues with the API data. First, before 2013, the monitoring stations responsible for collecting the data were run by local governments rather than being directly controlled by the MEP. The environmental bureaus responsible for gathering and reporting the API data were appointed by local governments, which can create conflicts of interest and compromise the reliability of the data. Studies have shown that there has been widespread manipulation of the API data in the past (Andrews, 2008; Chen et al., 2012; Ghanem and Zhang, 2014). Second, the API did

\footnotetext{
${ }^{6}$ The API is a numerical index that ranges from 0 to 500 , which is calculated by transforming the concentration of various pollutants $\left(\mathrm{PM}_{10}, \mathrm{SO}_{2}\right.$, and $\left.\mathrm{NO}_{2}\right)$ into a standardized scale. Individual APIs are first calculated for each pollutant, and the overall API is determined by taking the maximum of these pollutant-specific APIs. The "max" pollutant that determines the overall API for the day is called the "dominant pollutant." According to a study by Chen et al. (2012) that analyzed daily API data from 37 cities between 2000 and 2009, when the API is above $50, \mathrm{PM}_{10}$ is the dominant pollutant in $91 \%$ of cases, while $\mathrm{SO}_{2}$ is the dominant pollutant in $8.6 \%$ of cases. Although the dominant pollutant had shifted from $\mathrm{SO}_{2}$ to particulate matter (PM) in the $2000 \mathrm{~s}$, the government did not systematically collect $\mathrm{PM}_{2.5}$ measures before 2013. Therefore, the reported API did not incorporate $\mathrm{PM}_{2.5}$, very likely the most harmful pollutant that the public should care about.
} 
not incorporate data on $\mathrm{PM}_{2.5}$, which is believed to be a major contributor to air pollution in China, as the main sources of emissions have shifted from coal-fired power plants to a mix of sources including transportation and manufacturing. Third, the reported data is not real-time, as it is collected manually rather than through automatic process. The daily index covers the 24-hour period from 12 p.m. of the previous day to $12 \mathrm{p} . \mathrm{m}$. of the current day, which further contributes to the discrepancy between the reported levels and the public's perception of air quality. ${ }^{7}$

Without systematic pollution monitoring and reporting, government agencies and the media often characterized high pollution episodes as fog rather than smog. For example, on November 27, 2011, newspaper headlines and the China Meteorological Administration attributed dense fog in Beijing and northern cities as the reason for widespread flight delays and cancellations. However, the real cause of the low visibility was extreme pollution, as can be seen in Figure 1, which shows the aerosol optical depth (AOD) - a measure of atmospheric particulate pollution obtained through remote sensing - provided by NASA satellites. The average AOD concentration in China is typically around 0.5 (in the U.S., the average is usually between 0.1 to 0.15 ). On that day, the AOD value was 4.5 or higher in many northern cities. A similar pollution event that occurred on December 4-6, 2011 was again reported as dense fog by major news media, including China Central Television, the predominant state television broadcaster in Mainland China, and popular websites such as sina.com.

The lack of awareness of $\mathrm{PM}_{2.5}$ and the "fog-smog" confusion among the public and the media were reflected upon by the prominent journalist-turned-environmentalist Chai Jing in her high-profile documentary on China's air pollution titled "Under the Dome" released in February 2015: “... I go back and check the headline from that day's newspaper (on December 1st, 2004): 'Fog at Beijing Capital Airport Causes Worst Flight Delays in Recent Years.' We all believed that it was fog back then. That's what we called it... as a former journalist, I started to blame myself because for all these years I had been reporting stories on pollution all across the country, I always thought pollution was about mining sites and those places near factories spewing smoke plumes. I never thought the skies that we saw every day could be polluted." 8

\footnotetext{
${ }^{7}$ Due to the lack of reliable and publicly available air quality information in China, the U.S. Embassy in Beijing installed a rooftop air quality monitor in April 2008 in anticipation of the summer Olympics being held in Beijing. The embassy reported hourly $\mathrm{PM}_{2.5}$ levels on its Twitter account to advise U.S. citizens who were traveling or conducting business in China. U.S. consulates in Guangzhou, Shanghai, and Shenyang also followed suit in later years. However, Twitter is banned in China, so the data collected by the U.S. Embassy and consulates was not widely circulated among the general public in these cities, even though it has received attention from environmental activists.

${ }^{8}$ The documentary has been compared with Al Gore's An Inconvenient Truth in terms of its style and
} 
The 2013 Monitoring Program To effectively monitor local air pollution levels and to address the pitfalls of the previous reporting system (Greenstone et al., 2022), the MEP implemented a nationwide monitoring program starting in 2013. The focus was to build an efficient, scientific system to monitor air quality and to publicly disclose monitoring data in real-time. This monitoring program, the subject of our study, contained two major provisions.

First, it required the installation of a comprehensive network of monitors in 337 cities that in total covered an estimated $98 \%$ of the country's population. The program initiated continuous monitoring of all major air pollutants, including $\mathrm{PM}_{2.5}, \mathrm{PM}_{10}, \mathrm{O}_{3}, \mathrm{CO}, \mathrm{NO}_{2}$, and $\mathrm{SO}_{2}$. Figure 2 plots the rollout of the program. The rollout was implemented in three waves, and cities are assigned to waves based on administrative hierarchies and designated status that were determined long before the monitoring program. Cities in wave 1 include those in the Jing-Jin-Ji Metropolitan Region, the Yangtze River Delta Economic Zone, the Pearl River Delta Metropolitan Region, direct administered municipalities, and provincial capitals. They are required to start monitoring by December 31, 2012. Wave 2 contains cities that were among the Environmental Improvement Priority Cities or National Environmental Protection Exemplary Cities, both designated between 1997 and 2007. ${ }^{9}$ These cities were required to begin monitoring by October 31, 2013. Wave 3 includes all remaining cities which were required to monitor by November 30th, 2014. When the program was completed, the government had installed more than 1,400 monitoring stations nationwide. ${ }^{10}$

There are two nuanced aspects of the program rollout. First, there are a small number of cities that are assigned to waves that depart from the city hierarchy or designated status. For example, the city of Xiamen does not belong to any of the three special economic zones, is neither a self-administered municipality nor a provincial capital, but was in wave 1 . In Section 4.3, we show that a majority of these deviations are due to geographic contiguity. That is, they border cities in the corresponding wave and it makes sense administratively to group them into one wave. Second, as is often the case in China, the official rollout dates specified in the government guidelines serve as "deadlines" to implement the policy.

impact. The film had been viewed over 150 million times on the popular website tencent.com within three days of its release, before the government took it offline. Tu et al. (2020) show that household willingness to pay for clean air increased among those who had viewed the documentary.

${ }^{9}$ The Environmental Improvement Priority Cities were designated in 2007 during the 11th Five-Year period and contained important regional economic centers and cities that faced significant environmental challenges. The National Environmental Protection Exemplary Cities program was created during the 9th Five-Year Plan based on a host of environmental quality criteria.

${ }^{10}$ For the list of cities in each wave, see MEP's official documents 'Air Quality Monitoring Program Wave One Implementation Guideline' (May 21, 2012), 'Air Quality Monitoring Program Wave Two Implementation Guideline' (March 22, 2013), and 'Air Quality Monitoring Program Wave Three Implementation Guideline' (May 7, 2014). 
In practice, cities may finish installing monitors prior to the official rollout dates. We are unaware of reliable data that record the exact dates when cities finished installing monitors. To make progress, we manually search news media and city yearbooks for when pollution information first became available in each city, which we call "field" rollout dates. Our manual data collection effort yields fuzzy information at best. In Section 5.4, we show that our findings are robust to using the field rollout dates instead of official rollout dates.

Second, and more importantly, the monitoring network is coupled with a real-time information dissemination system. In contrast to the previous API reporting system, the MEP directly controls the newly established pollution monitors to avoid manipulation by the local government. All monitoring data are automatically streamed to MEP's information center and broadcast to the general public in real time on the MEP's website. Hourly and daily concentrations of $\mathrm{PM}_{2.5}, \mathrm{PM}_{10}, \mathrm{O}_{3}, \mathrm{CO}, \mathrm{NO}_{2}$, and $\mathrm{SO}_{2}$, as well as an Air Quality Index (AQI) encompassing all six pollutants, are available for each individual monitoring station and city. ${ }^{11}$ Appendix Figure E.1 provides a screenshot of the interactive website interface. Importantly, MEP allows private parties to access and stream data directly from its website. This has spurred a surge in media reports and mobile phone applications that display real-time air quality information. Section 3 provides more detail on the program's effects on information access and public awareness about $\mathrm{PM}_{2.5}$.

\section{$2.2 \quad$ Data}

We compile multiple data sets to allow for a comprehensive study of the impacts of the monitoring program on a variety of outcomes. The data sets include information on consumer awareness and online search behavior, public opinion toward environmental issues, bank card transactions, air purifier sales, mortality rates, and air quality measures from satellites. Table 1 shows city and time coverage as well as the mean and standard deviation of the primary variables used in our analysis. Our air purifier sales data cover 50 cities or about $28 \%$ of the national population. We draw public opinions on environmental issues from nationally representative panel surveys that are available for the years 2012, 2014, 2016, and 2018. All other data sets are national or nationally representative in scope and are available at the city-weekly level from January 2011 to April 2016, except for the mortality data which covers a slightly longer time span through December 2016. Below we provide more details on each individual data source.

\footnotetext{
${ }^{11}$ The AQI is on a scale of 0 to 500 and separated into six categories, with 0-50 (green) for "good air quality," 51-100 (yellow) for "moderate pollution," 101-150 (orange) for "unhealthy for sensitive groups," 151-200 (red) for "unhealthy," 201-300 (purple) for "very unhealthy," and 301 and higher (maroon) for "hazardous."
} 
Mass Media, Mobile Phone Apps, and Web Searches The high Internet and mobile phone penetration in China provides a unique opportunity to investigate pollution awareness using digital sources. ${ }^{12}$ We draw on several digital sources to illustrate the evolution of public access to pollution information. First, we look at the publication trends in the People's Daily, the government's official newspaper. We pull articles that contain the word "smog" (or "air pollution", or "atmospheric pollution") from the People's Daily's digital archive. We also identify the relevant cities mentioned in each of these articles.

Second, we scraped Apple's App Store to obtain release information on Chinese mobile apps that contain air pollution information in December 2015, using keywords including "smog," "air pollution," and "atmospheric pollution." 13 These apps typically display current hourly pollution levels; some also provide health-related guidelines (e.g., avoiding outdoor activities) when pollution levels are high. Appendix Figure E.2 is an example screenshot from a widely-used pollution app. We also scraped information on apps in other major categories including gaming, reading, and shopping, etc., which will help us tease out differential changes in the availability of pollution apps.

Third, the most widely used search engine in China, Baidu, provides search intensity indices that summarize the number of queries for certain words in a given city and on a given day among both desktop and mobile users since 2011. These search indices are generated using an algorithm similar to Google Trends. We focus on the search index for "smog," the buzzword for air pollution.

Public Opinion To complement the media data, we obtain survey data on public opinion from the China Family Panel Studies (CFPS), a nationally representative, bi-annual longitudinal survey of Chinese communities, families, and individuals. CFPS is one of the most widely used datasets to study public finance and labor markets in China, and is often seen as the Chinese equivalent of the Panel Study of Income Dynamics (PSID). Starting in 2012, CFPS asked respondents to state the perceived "seriousness" of a host of social and economic issues - including corruption, education, environment, housing, inequality, jobs, medical care, and social security - on a scale of 1 to 10, with 10 meaning "the most serious." We use the CFPS data from 2012, 2014, 2016, and 2018, which include 83,237 respondents living in 126 cities. Among these cities, 34, 39, and 49 are in the first, second, and third

\footnotetext{
${ }^{12}$ Data from the China Internet Network Information Center show that, by the end of 2012, China had about 0.56 billion internet users (or $40 \%$ of its population). More than $99 \%$ of Internet users have heard of Baidu, the most popular search engine (seconded by Google, $87 \%$ ), and $98 \%$ have used it in the past six months (seconded by 360.cn, 43\%). Mobile phone prevalence rose from 73.5 per 100 people in 2011 to 95.6 per 100 people in 2016 (National Bureau of Statistics), with a smart-phone penetration rate of $72 \%$ in 2013 (Nielsen).

${ }^{13}$ The query returned the 200 most relevant apps for a given keyword.
} 
wave of the monitoring program rollout, respectively.

Bank Card Transactions We measure shopping trips using the universe of credit card and debit card transactions from UnionPay, the only inter-bank payment clearinghouse in China and the largest such network in the world. The database covers $59 \%$ of China's national consumption and 22\% of its GDP in 2015 as shown in Appendix Figure E.3. For each transaction, we observe the merchant name and location, and transaction amount and time. Appendix Figure E.4 shows the spatial distribution of active cards and transactions. Credit and debit cards are widely adopted across the country as the most commonly used transaction method, especially in urban areas (Barwick et al., 2020). ${ }^{14}$ To the best of our knowledge, the fine degree of spatial and temporal resolution provided by this data set represents the most comprehensive data available on consumption activities for China.

Two features of the data are worth mentioning. First, the Point-of-Sale information in the UnionPay data allows us to distinguish between online and offline transactions. Our data contain a small fraction of transactions that are made online, which we drop because tracing these buyers' physical locations is difficult. ${ }^{15}$ We focus on the vast majority of the transactions that are made offline to measure outdoor purchase trips. Second, we do not observe specific items purchased in each transaction. Instead, UnionPay classifies merchants into over 300 categories, such as department stores and supermarkets. We use the category information in our analysis to measure the general purpose of each trip.

Our key outcome variable is the purchase frequency per card, defined as the number of transactions in a city-week per active card, where active cards are cards with positive transactions in a given city-year. We focus on a $1 \%$ random sample of cards and all of their associated transactions, with an average of 18.3 million active cards at any given point in time.

Air Purifier Sales To measure defensive spending, we acquire air purifier sales data from Growth from Knowledge, a leading market research firm, on the total units of air purifiers sold for both residential and institutional purposes. The data are available at monthly frequencies for 50 cities from 2012-2016. Together, these cities account for $28 \%$ of the national population. Among these 50 cities, 34, 11, and 5 are in the first, second, and third waves of the monitoring program rollout, respectively.

\footnotetext{
${ }^{14}$ Since 2016, mobile payment apps including Alipay and Wechat have become popular, but cards transactions still account for more than $50 \%$ of national consumption.

${ }^{15}$ Online transactions accounted for $5.1 \%$ of total transaction volume and $3.6 \%$ by transaction value between 2013 and 2015. The shares were smaller in earlier years.
} 
Mortality The Chinese Center for Disease Control and Prevention (CDC) operates a Disease Surveillance Points (DSP) system that covers 73 million individuals in 131 cities, a 5\% representative sample of China's population over the 2011-2016 period. Among these 131 cities, 38 implemented the monitoring program in the first wave, 38 in the second wave, and 55 in the last wave. ${ }^{16}$ The DSP mortality database, drawn from hospital records and surveys of the deceased's household, is one of the highest-quality health databases used in recent medical and economic research (Zhou et al., 2016; Ebenstein et al., 2017). We observe the number of persons and total deaths by each city-week-age group, and separately by causes of death.

Air Pollution To overcome the challenge that reliable pollution data are only available after the monitoring program, we obtain ambient air quality measures from AOD via the Moderate Resolution Imaging Spectroradiometer (MODIS) algorithm, operated by NASA and installed on the platform of the research satellite Terra. The original data have a geographic resolution of 10-by-10 $\mathrm{km}$ and a scanning frequency of 30 minutes, which we average to the city-week level from 2011 to 2016. MODIS records the degree to which sunlight is scattered or absorbed in the entire atmospheric column corresponding to the overpassed area under cloud-clear condition. As such, AOD captures concentration of particles such as sulfates, nitrates, black carbons, and sea salts, and serve as a proxy for outdoor particulate matter pollution (Van Donkelaar, Martin and Park, 2006). Appendix Figure E.5 documents a strong correspondence between $\mathrm{AOD}$ and $\mathrm{PM}_{2.5}$ after the implementation of the monitoring $\operatorname{program} .{ }^{17}$

\section{Information Access and Awareness}

In this section, we document how the monitoring program improved public access to pollution information and increased consumer awareness of air pollution. Unless noted otherwise, for

\footnotetext{
${ }^{16}$ The original DSP data report information for 161 counties and city districts which are smaller geo-units than cities. To use the same geographic unit of analysis throughout the paper, we aggregate the county/city district level data to the city level.

${ }^{17}$ An important advantage of the MODIS AOD measure is that it is available at high temporal frequency, which allows us to exploit weekly variation in air pollution to identify short-term responses of avoidance behavior and health outcomes. Most other remote-sensing measures of air pollution, such as the widely used Van Donkelaar et al. (2016), are provided at the annual level. MODIS AOD also uses a direct retrieval algorithm that infers air quality purely through optical properties of sunlight reflection and absorption; that MODIS AOD is free from complicated atmospheric and chemistry modeling helps alleviate concerns that the measurement itself may change over time in ways that might be correlated with the policy change. For example, MODIS AOD is not calibrated to ground $\mathrm{PM}_{2.5}$ monitoring data and therefore the measurement itself is not influenced by the change in the availability of $\mathrm{PM}_{2.5}$ data because of China's monitoring policy.
} 
each outcome we report (1) a national-level time series graph from January 2011 to April 2016, and (2) an event study graph at the city level from eight quarters before the monitoring program reached the city and five quarters after. ${ }^{18}$ Both graphs display raw data patterns with no control variables included, though the patterns are essentially the same with spatial or temporal controls as shown in Appendix Figure E.6. The coefficient for event quarter -1 (the quarter prior to the monitoring program) is normalized to 0. We report the $95 \%$ confidence interval constructed from standard errors clustered at the city level.

\subsection{Information Access: News and Mobile Apps}

News media and mobile phone apps are among the primary venues for the general public to access pollution information. In Panel (a) of Figure 3, we count the number of days in each month when the People's Daily mentions "smog" in any articles. "Smog" is rarely mentioned in the 1990s and 2000s. Almost immediately following the monitoring program's initial rollout, the frequency of "smog" appearing in the People's Daily jumped from two days per month in 2012 to sixteen days per month in 2013. It is possible that the initial increase in media coverage may have been due to coverage of the monitoring program itself. However, it is unlikely that this is the sole reason for the sustained high level of media coverage throughout the rest of the sample period. ${ }^{19}$

The national trend in media's smog mention may reflect changes in the general environment (e.g., shifts in government policies). To hone in on the impact of the monitoring program, we scan each smog-containing article in the People's Daily to determine the list of cities mentioned. This allows us to construct a city panel data set and, combined with the staggered rollout of the monitoring program, conduct an event study for changes in smog mentions frequency before and after a city begins to monitor pollution.

Panel (b) of Figure 3 provides an event-time plot of smog mentions as a function of quarters relative to a local city's monitoring program rollout, where smog mention is standardized to zero mean and unit variance. The graphical pattern features a discrete increase exactly on the rollout date (event quarter $t=0$ ). By one year after the rollout, smog mentions in cities with monitoring stations increased by $60 \%$ of a standard deviation $(p$-value $=0.038)$. Other keywords, such as "air pollution" and "atmospheric pollution," exhibited similar increases in media reports after the monitoring program.

\footnotetext{
${ }^{18}$ The event window is chosen to provide the longest balanced panel possible given the data available. The data covers the period from January 2011 to April 2016, and the wave $1 / 2 / 3$ cities received pollution information in January 2013/November 2013/December 2014, respectively. This allows us to examine 8/11/16 pre-policy quarters and 13/10/5 post-policy quarters for these cities.

${ }^{19}$ There was a modest increase in the frequency of the term "smog" before 2013. A close read of the articles indicates that these phrases were mostly used to describe dense fog and were rarely associated with pollution.
} 
Next, we examine the availability of mobile phone apps, which can provide users with real-time updates on air quality information, in contrast to newspapers that only provide daily updates. We compare the number of newly released pollution apps with that of other popular app categories, including gaming, music, video, reading, finance, sports, education, shopping, and navigation. As shown in Appendix Figure E.7, there is a clear surge in the number of pollution apps released after the monitoring program, relative to non-pollution apps. Among all pollution apps that were available for download in December 2015 (i.e., when we queried for the data), 82\% were released between 2013 and 2015, compared to 18\% that were released between 2009 and 2013. In contrast, $38 \%$ and $62 \%$ of the apps in the control group were released during 2009-2013 and 2013-2015, respectively.

\subsection{Awareness: Web Searches}

We measure the demand for pollution-related information by Internet keyword searches on Baidu. This analysis is analogous to the examination of "smog" news in Section 3.1. Again, we focus on search queries for "smog," though the patterns for keywords "air pollution," "mask," and "air purifier" are very similar.

Figure 4 repeats the exercise in Figure 3 but uses the search index as the outcome variable. Panel (a) of Figure 4 plots the time-series pattern of the search index for "smog" at the national level. The index varies between 0 and 200 for most of 2011 and 2012 and jumps overnight from 175 in December 2012 to 4,400 in January 2013, the month of the initial rollout. This is a 25-fold increase. The search index also began to exhibit a strong seasonal pattern after 2013, with the highest values occurring during the winter seasons. This mirrors the seasonality of smog, which tends to be more severe during the winter months due to the increased use of coal for heating. ${ }^{20}$ The search indices of 21,000 in December 2013 and 25,000 in January 2015, two outliers that were omitted from the graph for readability, correspond to the two worst smog episodes in the country's recent history.

Leveraging disaggregated search indices for over 300 cities, Panel (b) of Figure 4 provides an event-time plot of the standardized search indexes two years before and one year after the rollout in a local city. Echoing results in Section 3.1, the search index remains flat before the monitoring program and rises rapidly after monitoring starts. Within one year after the rollout, smog searches increase by on average $75 \%$ of a standard deviation. ${ }^{21}$

\footnotetext{
${ }^{20}$ We report correlation coefficient estimates in Appendix Table E.2.

${ }^{21}$ As pollution tends to peak during the winter months in China, we expect web searches to increase immediately after the monitoring rollout as well as 9-12 months after the rollout (the following winter). This is indeed what we find: media mentions of smog (panel (b) of Figure 3), web searches (panel (b) of Figure 4), and air purifier purchases (panel (b) of Figure 7) all exhibit similar patterns. Importantly, there were no spikes in these metrics 9-12 months before the monitoring rollout, indicating a lack of awareness in the
} 
The surge in Internet searches both nationally and locally and their strong correlation with air pollution after the start of the monitoring program provide compelling evidence that concepts such as "smog" and "air pollution," as well as related adverse health consequences, entered the public domain as a result of easy access to information provided by the monitoring program. As forcefully put by Wainwright (2014) and quoted by Greenstone and Schwarz (2018), "(by 2014,) the daily talk of the AQI has become a national pastime amongst ex-pats and Chinese locals alike. Air quality apps are the staple of every smartphone. Chinese micro blogs and parenting forums are monopolized by discussions about the best air filters and chatter about holidays to 'clean air destinations.' "

\subsection{Awareness: Public Opinion}

Public opinion toward environmental issues has also changed as a result of the monitoring program. Our analysis uses data from the China Family Panel Studies (CFPS), a nationally representative, bi-annual longitudinal survey of Chinese families and individuals. In 2012, the CFPS began including a set of questions that ask respondents to rate the perceived level of concern for various social and economic issues, including corruption, education, environment, housing, inequality, jobs, medical care, and social security, on a scale from 1 to 10. For our analysis, we utilize the CFPS data from the 2012, 2014, 2016, and 2018 waves, comprising 83,237 respondents living in 126 cities. Of these cities, 34, 39, and 49 are in the first, second, and third wave of the monitoring program rollout, respectively.

We investigate whether the monitoring program influenced concerns about the environment and other subjects by regressing the respondents' expressed level-of-concern metric on a post-monitoring dummy, while controlling for respondent fixed effects and year fixed effects. ${ }^{22}$ Panel (a) of Figure 5 shows the changes in the level of concern for all eight subjects. The results indicate that respondents had increased concerns about environmental issues after the monitoring program. In contrast, there were no statistically significant changes in respondents' views towards other economic and social issues.

absence of pollution information.

${ }^{22}$ Specifically, we estimate the following regression equation:

$$
\text { Concern }_{i c y}^{s}=\beta^{s} \cdot \operatorname{Post}_{c y}+\alpha_{i}^{s}+\alpha_{y}^{s}+\varepsilon_{i c y}^{s}
$$

where $\{s, i, c, y\}$ denotes ssubject, individual, city, year\}, and the equation is estimated separately for each subject $s$. The dependent variable Concern $_{i c y}^{s}$ represents the level of concern expressed by individual $i$ about subject $s$ on a scale of 1-10. The key regressor Post ${ }_{c y}$ is an indicator for city-years after the pollution information rollout. We assign 2014, 2016, and 2018 as the post period for cities in waves 1 and 2 of the monitoring rollout, and 2016 and 2018 as the post period for cities in wave $3 . \alpha_{i}^{s}$ and $\alpha_{y}^{s}$ are individual and year fixed effects, respectively. We weight all regressions by the individual-level survey weight to obtain nationally-representative estimates and cluster standard errors at the city level. 
In panel (b) of Figure 5, we further show that the increase in respondents' environmental concerns after monitoring policies are driven primarily by those live in cities with high levels of air pollution. Specifically, we relate a respondent's environmental concern with his/her city's 2015-2016 average $\mathrm{PM}_{2.5}$ concentration. ${ }^{23}$ We find that respondents' concerns about environmental issues are essentially uncorrelated (or slightly negatively correlated) with their cities' pollution levels before the monitoring program (shown by the circles); environmental concerns and pollution become strongly positively correlated after monitoring (shown by the triangles). That is, individuals living in cities with worse air quality become disproportionately more concerned with pollution than individuals living in clean cities.

To provide additional support for our findings, we also analyze data from the "Message Board" for government leaders, a government web portal that allows citizens to leave anonymous comments, including complaints and questions, to local government officials (Agarwal, Qin and Zhu, 2020). Our data includes over 1.3 million comments from 2010 to 2019. We use this data to count the number of air pollution-related comments (those that include "smog", "PM 2.5 ", "air pollution", or "air quality" in the text) over time. For comparison, we also look at comments related to water pollution issues (comments with "water pollution" or "water quality" in the content). Appendix Figure E.8 plots these trends. Air pollution related complaints rose sharply in 2013, which coincided with the launch of the monitoring program. No similar spike was observed for water pollution comments.

\section{Empirical Framework}

Our results so far demonstrate that the monitoring program significantly expanded access to pollution information and elevated households' awareness and concerns towards pollution issues. These changes may in turn influence the behavior of individuals who take steps to reduce the negative effects of pollution. In Appendix B, we formalize this idea using a stylized model that incorporates the elements of information economics (Hirshleifer, 1971; Hilton, 1981) into a classical model of health demand and production (Grossman, 1972; Harrington and Portney, 1987). In particular, we show that an information program that reduces the wedge between individuals' perceived and experienced air pollution can (a) increases pollution avoidance behavior and (b) reduces health damage from the same amount of outdoor pollution. The model also provides a framework to estimate the costs and benefits of the monitoring program which we come back to in Section 7. In this section, we describe

\footnotetext{
${ }^{23}$ Cities in different information rollout waves have different monitoring start dates. In particular, wave 3 cities did not start monitoring until the end of 2014. We therefore measure city's average pollution using 2015-2016 data so that pollution levels are consistently calculated based on the same time period for all cities.
} 
the econometric implementation of these theoretical predictions.

Our empirical objective is to examine whether the monitoring program has changed avoidance behavior and health effects of pollution. In the literature, these terms are typically measured using "pollution gradients", i.e., $\frac{\partial \text { Outdoor Activities }}{\partial \text { Pollution }}$ for pollution avoidance and $\frac{\partial \text { Health }}{\partial \text { Pollution }}$ for pollution's health damage. ${ }^{24}$ Our innovation to the literature is to evaluate how these pollution gradients change as the monitoring program drastically improves the public access to pollution information.

\subsection{Econometric Specification}

We estimate the changes in the outcome-pollution gradient using the following equation:

$$
\text { Outcome }_{c t}=\alpha \text { Pollution }_{c t}+\beta \text { Pollution }_{c t} \times \text { Post }_{c t}+\boldsymbol{x}_{c t}^{\prime} \gamma+\varepsilon_{c t},
$$

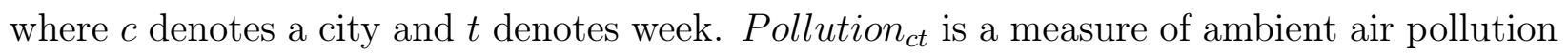
that equals the log of AOD in the city-week. The dummy variable Post ct $_{\text {represents the }}$ information treatment, and takes the value one for all periods after city $c$ implements the monitoring program. $\boldsymbol{x}_{c t}$ is a vector of control variables including the Post $t_{c t}$ term, city fixed effects, year fixed effects, week-of-year fixed effects, and city-specific time trends. ${ }^{25}$ Unless otherwise stated, all regression analyses and event studies control for these fixed effects. In robust specifications, we include more flexible controls such as week-of-sample effects and region-by-week-of-sample effects. $\varepsilon_{c t}$ is the error term. We cluster standard errors at the city level to allow for arbitrary serial correlations among the sample periods. In Equation (1), $\alpha$ represents the outcome-pollution gradient before the monitoring program, and $\beta$, the primary coefficient of interest, denotes changes in the gradient after the program. We defer the identifying assumptions underlying the causal inference of $\beta$ to Section 4.2.

For each outcome, we also implement an event study version of Equation (1) by replacing

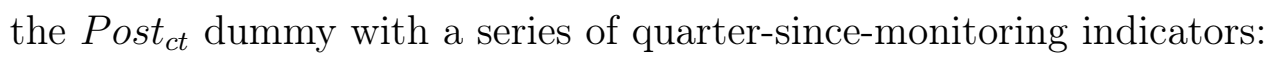

\footnotetext{
${ }^{24}$ Examples include Graff Zivin and Neidell (2009), Deschenes, Greenstone and Shapiro (2017), and Deryugina et al. (2019) which study the effects of pollution on outdoor activities, defensive drug spending, and mortality, respectively.

${ }^{25}$ City fixed effects control for all permanent difference of city characteristics in the cross section. Cityspecific time trends account for any differential trends in the outcome that may be related to the policy being studied, such as the differential rate of card penetration across cities in different waves. Controlling for city-specific trends is demanding and results in more conservative estimates (see Barwick et al. (2019), the original NBER Working Paper version of this paper, that does not include time trends controls). In unreported analysis, we have confirmed that other specifications of time trends, such as quadratic or cubic trends, or trends that are allowed to vary by pre- and post-monitoring periods, all produce similar results.
} 


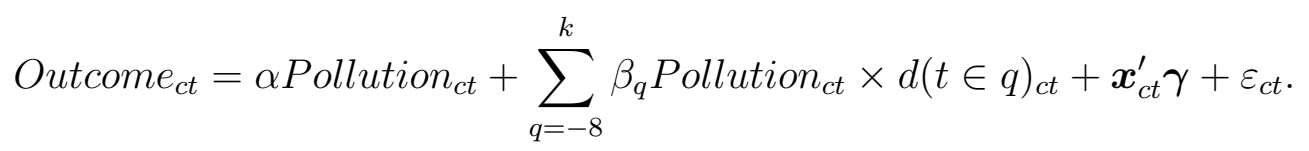

where $d(t \in q)_{c t}$ indicates week $t$ being in the $q^{t h}$ quarter since monitoring began in city $c .{ }^{26}$ We plot the estimates of $\beta_{q}$ 's to provide a visual representation of how the relationship between pollution and outcome, i.e., the outcome-pollution gradient, evolves as a function of time relative to monitoring rollout. Cities in different waves have different numbers of pre- and post-periods. Our event studies trace out the evolution of $\beta_{q}$ 's starting eight quarters before the monitoring rollout. Depending on data availability (Table 1), we report post-monitoring $\beta_{q}$ 's for five quarters in the analysis of purchase trips and air-purifier sales $(k=5)$ and eight quarters in the analysis of mortality $(k=8)$. These event time windows are chosen to ensure a balanced underlying sample for each coefficient. We dummy out sample periods outside of the event window. We use the same estimation framework as described in Equations (1)-(2) throughout the regression analysis below.

Equations (1)-(2) highlight the difference between our study and the previous literature that estimates the causal effect of air pollution exposure. Conventionally, the key threat to identification arises because pollution exposure is likely to be correlated with the error term: $\mathbb{E}\left(\right.$ Pollution $\left._{c t} \times \varepsilon_{c t}\right) \neq 0$. Such endogeneity could be due to omitted variables or measurement errors in pollution exposure. The scope of our empirical analysis differs in that, in most cases, we are not interested in the causal effect of pollution per se (which is $\alpha$ ), but rather in the change in the causal effect that takes place after the monitoring program (which is $\beta$ ). The key insight of our empirical framework is that, under reasonable assumptions, one can consistently estimate the change in pollution's causal effects $(\beta)$ using OLS, without having to consistently estimate the level of the effect $(\alpha)$. In Section 4.2 that follows, we discuss identification issues in more details.

\subsection{Identification}

We now describe the identification assumptions required to causally estimate $\beta$ using OLS estimation equation (1). Intuitively, if we were to separately estimate the outcome-pollution slope using data before and after the treatment, the endogeneity in pollution would lead to inconsistency in both estimates. However, if the nature of the endogeneity is not affected by the treatment, the inconsistency in the slope estimates could cancel out, leaving the OLS estimate of $\beta$ consistent. The following two assumptions formalize this intuition:

\footnotetext{
${ }^{26}$ We report quarterly (rather than weekly) event study coefficients to average out noise in the trends. This does not affect the estimation of the average program impact.
} 
Identification assumption 1: $\varepsilon_{c t} \perp$ Post $_{c t} \mid \boldsymbol{x}_{c t}$. This standard exogeneity assumption implies that conditioning on city attributes and other controls $\boldsymbol{x}_{c t}$ (e.g., city and time fixed

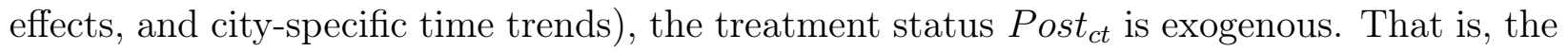
program rollout is as good as random conditional on the control variables.

Identification assumption 2: Post $_{c t} \perp$ Pollution $_{c t} \mid \boldsymbol{x}_{c t}$. This assumption implies that the treatment status is independent of pollution levels conditioning on $\boldsymbol{x}_{c t}$ (i.e., variations in pollution unexplained by $\boldsymbol{x}_{c t}$ ). The fact that the first-wave cities tend to have higher pollution levels on average does not violate this assumption per se as the average pollution levels can be controlled by city fixed effects. This assumption requires the treatment status being exogenous to the pollution variation unexplained by $\boldsymbol{x}_{c t}$. It ensures that the nature of the endogeneity in pollution does not change before and after the policy. ${ }^{27}$

We provide formal econometric arguments in Appendix C. Intuitively, there are two sources of inconsistency in the OLS estimate of $\beta$ in Equation (1): one from smearing due to

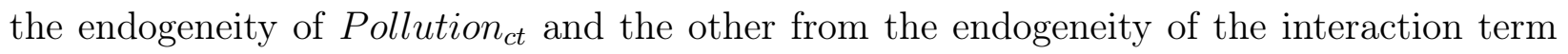
Pollution $_{c t} \times$ Post $_{c t}$. We show that inconsistency from these two sources cancels out - therefore leaving the OLS estimate of the difference (the change in the pollution gradient that we are interested in) to be consistent - under the two identification assumptions. Section 4.3 next presents empirical support of two identificaitons assumptions and discusses additional empirical challenges.

\subsection{Potential Identification Threats}

The identification of $\alpha$ and $\beta$, the parameters of interest in Equation (1), is based on the monitoring program's staggered rollout across cities. The two key identification assumptions outline above imply that the rollout schedule is not correlated with time-varying unobservables that could affect the outcome variables or pollution levels conditioning on the controls. Here we first examine the validity of these two assumptions. We also discuss potential estimation biases with a staggered rollout and adjustments for multiple-hypothesis testing as we examine multiple outcomes and subgroup heterogeneity - to reflect the best practices that are proposed in recent literature.

City Assignment to Monitoring Rollout Waves As the assignment of cities to different monitoring rollout waves is not random, here we investigate the consequences of this

\footnotetext{
${ }^{27}$ This assumption is stronger than what we need to prove the consistency of $\beta$ as shown in Appendix C.
} 
selection for our econometric analysis. First, the rollout wave assignment mainly corresponds to administrative hierarchies that are determined long before the policy, with a few exceptions due to exogenous factors such as geographic contiguity (Section 2.1). We present a visualization in Appendix Figure E.9. Colors indicate administrative hierarchies: deep blues are cities in the Jing-Jin-Ji Metropolitan Region, the Yangtze River Delta Economic Zone, the Pearl River Delta Metropolitan Region, direct-administered municipalities, and provincial capitals; light blues are 2007 Environmental Improvement Priority Cities and 1997-2007 National Environmental Protection Exemplary Cities; whites are all other cities. If rollout follows strictly administrative hierarchies, then all deep blue cities should be in wave 1, all light blue cities should be in wave 2 , and all white cities should be in wave 3 . This is true $93 \%, 88 \%$, and $86 \%$ of the time for wave 1, 2, and 3. Among the exceptions, we calculate that $55 \%, 69 \%$, and $100 \%$ of the cases (or $68 \%$ of total cases) are due to the fact that they border cities in the corresponding wave and it makes more sense administratively to group them into those waves.

Second, following Almond, Li and Zhang (2019), in Appendix Section D.1, we present a more formal, "zero-stage" analysis on the determinants of city rollout assignment. The idea is that, if the rollout wave assignment is mostly driven by pre-determined administrative hierarchies and status, then conditional on these characteristics, one should expect little explanatory power from additional controls, such as pollution or economic conditions. Results in Appendix Table E.3 confirm this. Conditioning on administrative hierarchies, levels especially trends in cities' pre-policy air pollution or income provide little to no additional explanatory power for city wave assignment, consistent with Identification assumptions 1 and 2 of Section 4.2 .

Third, Appendix Table E.4 summarizes economics and environmental attributes for cities in each wave. Cities in earlier waves tend to have a larger population, higher income, and higher levels of air pollution and industrial emissions. We note that our regression analyses include city fixed effects, and therefore permanent differences in city characteristics do not pose threats to our identification. In Appendix Table E.5, we further test whether time-varying city characteristics correlate with policy rollout after controlling for the suite of fixed effects controls we use in Equation (1). We examine a rich set of characteristics including pollution levels, local political and regulatory environment (e.g., the number of local officials ousted during the anti-corruption campaign, demographics of local political leaders, news mentions of regulation policies), and healthcare access (the number of medical facilities). Conditioning on the suite of fixed effects, the program rollout is not correlated with these city attributes. These "balancing checks" suggest a lack of city-level confounders that correlate with the timing of policy rollout, supporting Identification assumptions 1 and 
2 of Section 4.2.

Finally, the event study as outlined in Equation (2) helps diagnose potential endogeneity in rollout timing through pre-existing trends in the outcome of interest. The event studies in Figures 6 and 8 provide further support for Identification assumption 1 of Section 4.2.

Concurrent Policies as Potential Confounders Since 2014, the Chinese government has implemented a variety of environmental and energy programs as part of its efforts to combat air pollution, as noted in Greenstone et al. (2021). Here, we discuss several major policies and their potential to interfere with our analysis of the impact of the monitoring program. First, China rolled out pilot $\mathrm{CO}_{2}$ cap-and-trade programs targeted at heavily polluting industries in two provinces (Guangdong in December 2013 and Hubei in April 2014) and five individual cities (Shenzhen in June 2013, Beijing and Shanghai in November 2013, Tianjin in December 2013, and Chongqing in June 2014). ${ }^{28}$ The second policy is the energy reduction plan among over 16,000 of the largest energy users that collectively accounted for over $60 \%$ of national energy consumption in 2010. The policy started in 2012 and aimed to reduce energy intensity and carbon intensity as outlined in the 12th FiveYear Plan. The third policy is fuel switching from coal to natural gas for winter heating in northern China from the winter season of 2013, as part of the Action Plan on Air Pollution Prevention and Control.

Because the geography and timeline of these concurrent environmental policies do not significantly overlap with the monitoring program that we study in this paper, we expect the impact of these policies to be captured by the extensive set of spatial and temporal controls in our econometric models (e.g., city fixed effects and region-by-week-of-sample fixed effects). As shown in Appendix Table E.5, the monitoring program is not associated with any significant changes in pollution levels after controlling for fixed effects. This is supportive evidence, as if these other environmental policies were systematic confounders, we would expect our model to show an improvement in air quality due to the monitoring program. $^{29}$

\footnotetext{
${ }^{28}$ These seven regional pilots were a proof of concept for the national $\mathrm{CO}_{2}$ cap-and-trade program slated to come online in 2020. However, the program had a limited impact on carbon emission and air quality given the generous allocation and the lack of a strong punishment mechanism for noncompliance (Zhang, Wang and $\mathrm{Du}, 2017)$.

${ }^{29}$ It is important to clarify that our findings are not contradictory to the fact that China's overall air quality has significantly improved since 2015, due in part to various environmental regulations mentioned above (e.g., Greenstone et al., 2021). These trends are absorbed in our flexible city and time fixed effects. Our results show that, conditional on these fixed effects, the monitoring policy itself does not have an independent effect on improving air quality.
} 
Bias in Staggered DID Recent literature points out that the two-way fixed effects model in a staggered difference-in-differences (DID) setting could yield biased estimates when there are heterogeneous treatment effects over time or across units (De Chaisemartin and d'Haultfoeuille, 2020a,b; Sun and Abraham, 2020; Callaway and Sant'Anna, 2020; GoodmanBacon, 2021). One reason for this is that in two-way fixed effects DID models, treated units that are treated earlier serve as "controls" for later treated units. However, if there are heterogeneous treatment effects across units or over time, the treatment can cause units to be on different trends. This can make the post-treatment periods for earlier treated units no longer a valid comparison for later treated units, potentially leading to biased estimates.

Our research differs from previous studies in that we focus on the impact of the monitoring program on economic- and health-pollution gradients, rather than on the outcomes themselves. As a result, the standard solutions proposed in the literature do not directly apply to our study. However, to evaluate the potential for bias, we follow the intuition outlined in Goodman-Bacon (2021) and assess the robustness of our findings by restricting comparisons to "switcher" units and "not-yet-treated" units. Because our study involves three rollout waves, there are a limited number of such comparisons. We examine each of them individually and report robustness results in Sections 5.4 and 6.3.

Multiple Testing Adjustment In both of our analyses on card transactions and mortality, we are interested in subgroup heterogeneity - for example, whether the monitoring program generates differential impacts for deferrable versus nondeferrable transactions or the elderly versus non-elderly population. Since we examine multiple outcomes, we control for the family-wise error rate, i.e., the probability of incorrectly rejecting one or more null belonging to a family of hypotheses. Following the best practices as recommended in Aguilar-Gomez et al. (2022), we report the family-wise adjusted $p$-values for the $\beta$ coefficients based on 100 bootstraps of the free step-down procedure of Westfall and Young (1993), with standard errors clustered at the city level. Specifically, we first define an exclusive family of hypotheses for both card transactions and mortality. Each family contains the overall category and all subgroup categories. ${ }^{30}$ We implement the Westfall-Young procedure using the algorithm provided by Jones, Molitor and Reif (2019) and conduct the block bootstraps simulations at the city level.

Causal Effect of Pollution The main goal of our study is to estimate how the monitoring program changes the pollution gradient. Our key econometric insight is that, under

\footnotetext{
${ }^{30}$ In other words, we define a family as a column in Tables 2, 3, and 4. For example, for card transactions, a family is a column in Table 2 that includes the overall, deferrable, and nondeferrable transactions.
} 
reasonable and testable assumptions, this parameter can be causally identified by estimating the $\beta$ coefficient in Equation (1) using simple OLS. An alternative approach is to estimate the causal impacts of air pollution separately for periods before versus after the implementation of the monitoring program. We report this analysis in Section 6.3 using instrumental variables (IV) estimation strategies from the literature on the health effects of air pollution.

\section{Pollution Information and Behavioral Responses}

\subsection{Avoidance Behavior}

We first examine how the monitoring program changes household avoidance behavior. We use the bank card transaction data to build a measure of shopping trips, defined as the number of transactions in city $c$ at week $t$ per 10,000 active cards. As we explained in Section 2.2, we focus on transactions that take place in physical stores, which enables us to track outdoor activities. We use this measure of shopping trips as the outcome variable in Equation (1), and therefore the $\beta$ coefficient represents the change in the shopping trip-pollution gradient $\frac{\partial \text { Outdoor Activities }}{\partial \text { Pollution }}$ due to the monitoring program, reflecting avoidance behavior.

Figure 6 shows an event study graph for $\beta_{q}$ 's, with the coefficient for the event quarter -1 $\left(\beta_{-1}\right)$ normalized to zero. Two patterns emerge. First, before the program, the $\beta_{q}$ 's estimates are flat and also statistically indistinguishable from zero, suggesting a lack of pretrends. Second, the purchase-pollution gradient became negative almost immediately after the rollout of the monitoring program. Doubling the pollution readings leads to about 11 fewer trips per 10,000 cards on a weekly basis.

We examine the statistical precision and the robustness of these patterns in panel (a) of Table 2. Column 1 controls for city, week-of-year, and year fixed effects, as well as city-specific time trends, which correspond to the specification of Figure 6. Column 2 also includes week-of-sample fixed effects (fixed effects for all weeks during 2011-2016), exploiting variation in pollution across cities in the same week. Column 3 further adds region-by-year fixed effects, allowing for common trends in transactions and pollution that are specific to each region. ${ }^{31}$ Column 4 is our most stringent specification, controlling for region-byweek-of-sample fixed effects in addition to city fixed effects and city trends. We obtain similar results across the board. The parameter estimate on the interaction term suggests that when pollution doubles, weekly card transactions would decrease after the program by 12 more per 10,000 cards than before the program, representing a 1.4 percentage points

\footnotetext{
31 "Region" is a conventional partition of cities by location: north (36 cities), northeast (38 cities), east (105 cities), central south (81 cities), southwest (54 cities), and northwest (52 cities).
} 
decrease from the mean transaction frequency of 869.1 transactions per 10,000 cards. In other words, the shopping-pollution elasticity becomes 1.4 percentage points more negative post the monitoring program.A simple derivation shows that the program reduced card transactions by 1.4 percent, which we will use to calculate the welfare loss from foregone consumption in the cost-benefit analysis.

The observed effect is economically significant. As a point of reference, Cutter and Neidell (2009) find that when a "Spare the Air" alert is issued in the San Francisco Bay Area, daily traffic falls by 2.5-3.5 percent with the largest effect during and just after the morning commuting period. ${ }^{32}$ Graff Zivin and Neidell (2009) estimate that a one-day smog alert issued in Southern California leads to an 8-15\% reduction in attendance at two major outdoor facilities (the Los Angeles Zoo and the Griffith Park Observatory) on the same day, though the effect dissipates quickly in consecutive days of such alerts. While these two studies focus on daily behavioral changes after government-issued air quality warnings, our analysis focuses on changes in shopping behavior at the weekly level, which naturally accounts for within-week intertemporal substitution. Additionally, our data covers all consumption categories and represents $59 \%$ of national consumption.

Table 2 also shows that shopping trips are not affected by pollution before the program, as indicated by the insignificant coefficients for the main "Log(Pollution)" term. While our purchase-pollution slope estimate prior to the monitoring program may not be causal (as previously discussed), this finding is consistent with the idea that households did not take effective mitigating measures in the absence of information about pollution levels. We discuss this issue in more detail in Section 5.3 below.

Subcategory Spending We expect pollution avoidance behavior to be more relevant for consumption trips that can be reasonably delayed. In panels (b) and (c) of Table 2, we examine heterogeneity between "deferrable" and "nondeferrable" consumption. We define deferrable consumption as transactions that occurred in supermarkets, restaurant dining, and entertainment merchants. As expected, the monitoring program generates a steeper response among deferrable transactions where doubling pollution leads to a 2.6 percentage points reduction (Column 4), which explains about $74 \%$ of the change in the overall shoppingpollution gradient. The estimated effects on the rest of the consumption categories ("nondeferrable") are negative but not statistically significant.

This pattern of subgroup heterogeneity stands up to multiple hypothesis testing adjustments. As explained in Section 4.3, we used the method described in Westfall and Young

\footnotetext{
${ }^{32}$ The "Spare the Air" (STA) advisories, designed to elicit voluntary reductions in vehicle usage and encourage the usage of public transit and carpooling, are issued on days when ground-level ozone is predicted to exceed National Ambient Air Quality Standards.
} 
(1993) to calculate family-wise adjusted $p$-values, which are reported in brackets in Table 2. The results suggest both responses of overall and deferrable transactions are statistically significant.

While we have classified shopping trips as deferrable or non-deferrable in an effort to identify the categories most affected, this classification may seem somewhat arbitrary. In Appendix D.2, we describe an alternative, data-driven method to identify transactions that are more "outdoor" in nature. We reiterate that our analysis only includes transactions that occur in physical stores, which by definition involve some level of outdoor activities. However, there are some types of spending activities, such as visiting recreation parks, that may involve a longer time spent outside and potentially more pollution exposure compared to activities like shopping in malls. Specifically, we examine how precipitation shocks affect transaction activities across 273 narrowly defined merchant categories. The idea is that categories that are sensitive to precipitation events may be more likely to involve outdoor activities or be more easily deferred, and therefore may also be the most affected categories when individuals are trying to avoid air pollution. We relegate full details to Online Appendix D.2 (with results summarized in Appendix Figure E.10). Here we highlight several key findings.

First, the data-driven exercise appears to detect deferrable and/or outdoor trips successfully. The most precipitation-sensitive categories include snow car rentals and dealers, vacation houses, sports stadiums, amusement parks, resort areas, and garment stores, among others that can potentially be postponed or typically take place in open-air settings. ${ }^{33}$ Second, we conducted the pollution avoidance analysis again using shopping trips from the top 20 most precipitation-sensitive transaction categories as the dependent variable. Our results show that doubling the pollution concentration would lead to 5.5 percent fewer transactions after the monitoring rollout, indicating a larger response compared to the overall consumption gradient change we observed in panel (a) of Table 2.

\subsection{Defensive Spending}

In addition to reducing outdoor trips during times of high pollution, households may also increase "defensive" spending that directly reduces their pollution exposure. We examine purchases of air purifiers, which are popular household appliances in China used to mitigate the effects of severe air pollution (Ito and Zhang, 2018).

We obtain monthly sales of air purifiers for 50 large cities in China between 2012 and 2016 (Section 2.2). Panel (a) of Figure 7 plots the raw monthly time series of total units

\footnotetext{
${ }^{33}$ Additionally, it is reassuring to find that transactions for supermarkets, restaurant dining, and entertainment - which we identified as deferrable in Table 2 (b) - are disproportionately deferrable and outdoor in nature from a precipitation-sensitivity perspective.
} 
sold. Air purifier sales more than doubled and rose from 11,000 units per month in 2012 to over 25,000 units per month after 2013. The change in air purifier sales patterns appears to mirror the changes in web search behavior shown in Figure 4, with strong seasonality in air purifier sales emerging after 2013, often with large spikes in sales during the winter months. At the city level, similar to the pattern we see in web searches, the increase in air purifier sales coincides with the timing of the monitoring program rollout and doubles within four quarters of the rollout (panel (b) of Figure 7). Further evidence that the relationship between pollution and air purifier sales (the purchase-pollution gradient) became stronger after the rollout of the monitoring program is provided in Appendix Table E.6, which shows that sales became more positively correlated with month-over-month variations in air pollution after the availability of pollution information.

\subsection{Private Information and Avoidance}

The monitoring program resulted in the widespread dissemination of public information about air pollution. Individuals may also use private information to respond to pollution, such as visual or olfactory cues. In this subsection, we investigate the role of two sources of private information - air visibility and data from the U.S. Embassy - in pollution avoidance. In particular, we examine whether these sources of private information helped households avoid pollution before the monitoring program was implemented. A summary of our findings is provided here, with further details in Online Appendices D.3 and D.4.

The role of air visibility In Appendix D.3, we assess the possibility that people used air visibility as a proxy for pollution levels before the pollution information program. We report three main sets of findings. First, Panel (b) in Appendix Figure E.12 shows that while visibility is correlated with ambient pollution levels, the relationship has a large spread and is particularly noisy when visibility is poor. The pattern underlies challenges that people face in trying to avoid pollution without actual pollution monitoring data: both heavy fog and heavy smog could cause poor visibility outdoors.

Next, we re-estimate Equation 1 and use poor visibility as the key right-hand-side variable instead of pollution. Panel (c) in Appendix Figure E.12 shows that before the monitoring program, there were few card transactions during weeks with the visibility in the lowest qintiles. However, the shopping-visibility relationship is non-monotone, economically modest, and imprecisely estimated. The reduction in card transactions during weeks with the lowest visibility may reflect a genuine concern for poor visibility per se (e.g., road safety concerns) instead of pollution. 
More importantly, we find that adding actual pollution (measured with AODs) as control variables has little impact on the estimated shopping-visibility relationship before the program as shown in Panel (c) in Appendix Figure E.12. If people were using visibility as an indicator of pollution levels, we would expect the relationship between shopping and visibility to change once we directly control the pollution levels in the regression. This is indeed what we observe after the monitoring program as shown in Panel (d) of Appendix Figure E.12: the negative relationship between poor visibility and transactions attenuates once we control for pollution. These findings suggest that households were unlikely to have been using visibility as a means of effectively avoiding pollution before the monitoring program.

U.S. Embassy data The U.S. Embassy in Beijing installed a rooftop air quality monitor in April 2008 before the upcoming summer Olympics and started reporting hourly $\mathrm{PM}_{2.5}$ levels via its Twitter account (@beijingair) to advise U.S. citizens who travel and do business in China. U.S. consulates in Chengdu, Guangzhou, Shanghai, and Shenyang followed the practice in later years. Among the five cities with U.S. Embassy and Consulates, Beijing is the only city with independent $\mathrm{PM}_{2.5}$ data dating back to 2011. We therefore focus our analysis on Beijing and modify Equation (1) to test to examine whether the $\mathrm{PM}_{2.5}$ monitoring conducted by the U.S. was an effective source of information for pollution avoidance by local residents (see Appendix Section D.4 for more details).

Appendix Figure E.13 summarizes the results. Both weekly web searches for smog and card transactions exhibited a precisely-estimated, close-to-zero correlation with embassyreported air pollution before 2013. Analogous to our main findings, after 2013, the correlation between web searches and embassy-reported pollution becomes significantly positive, and the correlation between card transactions and pollution becomes significantly negative. The fact that the correlations were close to zero before 2013 and then increased significantly after 2013 suggests that the U.S. Embassy $\mathrm{PM}_{2.5}$ data were not a significant source of information for Beijing residents prior to 2013, when public $\mathrm{PM}_{2.5}$ data were not available. This is likely because Twitter was banned in China and the embassy data were not widely distributed among the general public, despite attracting attention from environmental activists. ${ }^{34}$

\footnotetext{
${ }^{34}$ U.S. Embassy's pollution data can serve as important information source where residents have access to it. Jha and Nauze (2022) show that the installation of U.S. Embassy air pollution monitoring in 50 cities around the world and the real-time Tweets of air quality information led to increases in local pollution awareness, as captured by Google Searches for terms related to air pollution.
} 


\subsection{Additional Robustness Checks}

Robustness Specifications Appendix Table E.7 examines the robustness to sample selections, alternative variable definitions, and additional controls. The first row of Table E.7 excludes the top $10 \%$ of the cities with the highest number of officials who were ousted as a result of China's sweeping anti-corruption campaign, which has been associated with a reduction in luxury consumption (Qian and Wen, 2015). The second row adds controls for the share of card transactions that occurred through online venues, as people might substitute offline trips with online purchases. The third row adds weather controls, including weekly average temperature, precipitation, wind speed, barometric pressure, and their interactions. The fourth row replaces the weekly average AOD readings with the maximum daily AOD value observed during the week. In the fifth row, we re-define the pre and post monitoring periods based on the "field" rollout dates that we manually collected from news media and city government yearbooks (Section 2.1). Appendix Figure E.14 plots the distribution of these dates. We do not use these field dates in our preferred specification because there may be significant measurement errors, such as delays or rounding of dates in news reporting. For example, many cities in wave 3 describe the start of the monitoring program generally as "starting in 2015," while the actual start date is likely in December 2014 according to the policy deadline. Lastly, we aggregate the data and conduct analysis at the quarterly frequency to capture avoidance effects that may manifest throughout a longer time horizon and to account for potential intertemporal substitution of consumption across different weeks of the quarter.

These robustness tests produce estimates that are similar across the board and generally indistinguishable from one another. The quarterly analysis on the last row produces a larger effect estimate than would be implied by a linear scale-up of the weekly estimate. This suggests pollution avoidance may extend beyond a weekly horizon, although some of the quarterly estimates are imprecise due to a 13 -fold reduction in the sample size.

Spatial Spillovers Appendix Table E.8 examines potential spatial spillover effects, e.g., whether new coverage about pollution episodes in one city raises awareness in nearby cities that do not have pollution monitoring yet. For each wave of treated cities, we introduce a group of neighboring cities that share borders with the treated cities but have not installed monitoring stations. These neighboring cities are assigned the same rollout time as their treated counterparts. We allow the changes in the shopping-pollution gradient to differ between the treated and neighboring cities. ${ }^{35}$ There are two key findings. First, there are no

\footnotetext{
${ }^{35}$ We incorporate two interaction terms: "Log(Pollution) $\times 1$ (after monitoring)" that captures changes in neighboring cities and " $\log ($ Pollution $) \times 1$ (after monitoring $) \times 1$ (Treated)" that capture additional changes
} 
detectable changes in the shopping-pollution gradient in neighboring cities at the time when the monitoring program began in the treated cities. Second, the shopping-pollution gradient change in treatment cities relative to that of their neighbors is similar in magnitude to the main effect reported in Table 2, though the triple interaction term is estimated with less precision than the main specification. The lack of spatial spillover of one city's information rollout to its neighbor cities suggests that having actual data about local pollution levels is important in shaping avoidance behavior.

Staggered Rollout As discussed in Section 4.3, to assess the potential bias due to staggered treatment, we follow the intuition laid out in Goodman-Bacon (2021) and only compare treated cities and not-yet-treated cities. Specifically, we conduct three separate analyses and compare cities in wave one with cities in the latter two waves, cities in wave one with cities in wave three, and cities in wave two with cities in wave three. We only include data from periods when the control group cities have not yet started pollution monitoring. Panel (a) of Appendix Figure E.15 illustrates the sample cities and periods for each analysis. The point estimates in Panel (b) of Figure E.15 vary across columns (for example, it is smaller in the specification that compares wave 2 cities with wave 3 cities), though primarily due to differences in the means of the dependent variable. The shopping-pollution gradient using different comparison groups ranges from $1 \%$ to $1.8 \%$, similar to our baseline findings.

\section{Pollution Information and Mortality Benefit}

\subsection{Mortality Responses}

Our previous analyses have documented changes in pollution information and awareness as well as a range of behavioral responses to the monitoring program. Our endpoint analysis examines whether the same amount of pollution exposure is associated with fewer deaths after pollution information becomes widely available as a result of risk-compensating behavioral responses. Our analysis uses weekly mortality data in 131 cities from 2011 to 2016.

Figure 8 plots the $\beta_{q}$ coefficient estimates for the event study described by Equation (2), where the dependent variable is the log of mortality rates. The mortality-pollution elasticity exhibits a flat trend before the program, followed by a noticeable decline afterward, consistent with the event study analysis on behavioral changes above. This decline appears to strengthen after a few quarters, suggesting that the mortality effect tends to manifest over

in treated cities. Cities in the last wave of the rollout are excluded from the analysis. Including these cities as treated units without neighboring pairs produces similar results. 
time. Table 3 reports the mortality analysis using Equation (1), with the same specifications as in Table 2. Panel (a) examines the mortality of all causes. The monitoring program leads to a significant 1.3-2.3 percentage points reduction in the mortality-pollution elasticity. That is, pollution became less deadly after the program: doubling pollution is associated with 1.32.3 percent fewer deaths after the program.

\subsection{Heterogeneity}

Cause- and Age-Specific Mortality Responses We continue our mortality analysis by examining subgroup responses. Panels (b) to (d) of Table 3 breaks drown overall mortality by the underlying causes of death. Panel (b) focuses on cardiorespiratory mortality and shows a 2.4-3.1 percentage point decrease in the mortality-pollution elasticity. Panel (c) shows that the effect on non-cardiorespiratory mortality is smaller and statistically insignificant. During our study period, cardiorespiratory deaths account for about half of total deaths. These estimates imply that about $80 \%$ of the reduction in mortality damage after the monitoring program comes from cardiovascular and respiratory causes which are widely considered as the most direct consequences of pollution exposure. Panel $(\mathrm{d})$ performs a placebo test that examines mortality that is associated with external injuries. There is no evidence that the monitoring program reduces injury-related mortality, as expected.

Table 4 reports the mortality effect for four age groups: infants, age 1-14, age 15-59, and age 60 and above. ${ }^{36}$ The effect of the monitoring program concentrates among the preteen and the elderly who are more vulnerable to pollution exposure than mid-aged adults. Interestingly, we find no change in the mortality-pollution relationship for infants under one year of age. This could be associated with the low pollution exposure among infants due to the traditional Chinese practice of keeping newborns indoors within the first few months of their birth to minimize outdoor exposure.

In Table 3 and Table 4, the numbers in brackets report the Westfall and Young (1993) family-wise adjusted $p$-values using standard errors clustered at the city level. For each table, a family of hypotheses includes the outcomes in all panels for each of the four types of fixed effects specifications. The coefficient of interest " $\log ($ Pollution $) \times 1$ (after monitoring)" survives multiple testing adjustment at the $5 \%$ significance level for cardiorespiratory mortality (Table 3, panel (b)) and for elderly mortality (Table 4, panel (d)). The family-adjusted p-value for the all-cause mortality effect (Table 3, panel (a)) varies from 0.006 to 0.134.

\footnotetext{
${ }^{36}$ The low number of observations for the infant and preteen analysis (Panels (a) and (b) of Table 4) is due to city-weeks with zero deaths.
} 
Mortality Responses and City Characteristics We further conduct heterogeneity analysis which provides suggestive evidence on the mechanism underlying the mortality effect. Specifically, we repeatedly split the sample into two using the average value of a series of city-level characteristics, including per capita income, share of the urban population, per capita number of hospitals, per capita residential electricity use, and share of mobile phone users. Results in Panel (b) of Table E.11 suggest a larger reduction in mortality damage in cities that experience a higher mobile phone penetration, consume a higher rate of residential electricity, and have more hospitals. Cities that have higher per capita income and are more urban also report a larger mortality reduction, though the estimates are noisier. These findings are consistent with the observation that residents in above-median cities are better able to engage in defensive spending to reduce the health damage from air pollution exposure, and hence benefit more from pollution information.

In Panel (a) of Table E.11, we repeat the heterogeneity analysis for shopping trips using the same set of city attributes. The overall patterns of the two panels appear consistent: residents in above-median cities exhibit stronger (though imprecisely estimated) avoidance behavior to elevated pollution. While there are various ways to protect oneself from the harmful effects of pollution, the data suggests that avoiding outdoor activities may be a short-term strategy for reducing the harm caused by pollution..

\subsection{Robustness Checks}

Instrumental variables approach Our econometric arguments in Section 4.2 and Appendix $\mathrm{C}$ show that one can causally estimate changes in the effects of pollution on outcomes ( $\beta$ in Equation (1)) using simple OLS under reasonable identification assumptions, without having to causally identify the level of the pollution's effects ( $\alpha$ in Equation (1)). These said, the levels of the effects, both before and after the monitoring rollout, could nevertheless be of academic and policy interest.

We present an instrumental variables (IV) strategy where we isolate plausibly exogenous variation in a city's local air pollution that is attributable to transported pollutants from upwind cities.(e.g., Barwick et al., 2020). We then estimate two-stage least squares (2SLS) regressions of mortality on air pollution, instrumenting pollution with the IV variable. We run the 2SLS regressions separately for periods before monitoring and after monitoring.

Appendix D.5 discusses in detail the IV construction and empirical results. Reassuringly, the changes in the mortality impact of air pollution (the mortality-pollution gradient) before and after the program are virtually the same whether the estimates are based on OLS or from IV regressions. This evidence supports the key identification assumption underlying 
the change-in-gradient analysis of Equation (1). The analysis also produces causal estimates on pollution's mortality impact for different underlying causes of death, separately for before and after the monitoring program.

Synthetic Control Analysis An alternative way to assess the impact of the monitoring policy is to examine changes in mortality outcomes directly, rather than changes in mortalitypollution gradients. If changes in pollution's effects are large enough across the spectrum of pollution levels, one could in principle detect an overall reduction in mortality rates. The key to this exercise is to identify a set of "control" cities for each treated city that can serve as reasonable counterfactuals in the absence of the monitoring rollout. We adopt the synthetic control method proposed by Abadie, Diamond and Hainmueller (2010) and Arkhangelsky et al. (2021). Following best practice, we pair each treated city with control cities that have not yet been exposed to the monitoring program and exclude wave three cities (those in the last wave without "not-yet-treated" controls). We calculate synthetic weights using data up to one year prior to the rollout of information and use the latter half of the pre-treatment period as a validation sample to evaluate the synthetic control method. We summarize our findings here and relegate details to Online Appendix Section D.6.

Panel (a) of Appendix Figure E.18 shows an event study for the synthetic analysis. Several patterns emerge. First, there are no obvious trends in the mortality differences between treated cities and synthetic control cities prior to the monitoring rollout, which validates the synthetic analysis. Second, relative to control cities, treated cities' mortality rates decrease by 1.64 percent on average after the monitoring program, though the drop is imprecisely estimated. Third, the reduction in mortality rates seems to be more concentrated during heavily polluted periods (highlighted by the gray areas on the chart) following the introduction of the monitoring program, a pattern that was not previously apparent. These patterns suggest that the imprecise average effect of the monitoring program may be masked by important heterogeneity.

Panel (b) of Appendix Figure E.18 explores this heterogeneity more formally by allowing the monitoring program's mortality effect to differ across quintiles of pollution levels. The program has no discernible impact on the mortality rate during periods of low pollution levels, but reduced mortality significantly (both statistically and economically) during high pollution periods. For example, the mortality rate decreases by nearly four percent in weeks with the 20 percent highest pollution levels. Importantly, this result is isomorphic to our gradient analysis in Section 6.1, where we document a negative coefficient for the pollution and post-treatment interaction. In theory, this negative relationship documented in Section 6.1 could be driven by an increase in mortality during low pollution periods, a decrease in 
mortality during high pollution periods, or some combination of both. The analysis here shows that our main finding of the monitoring program's mortality benefit is mostly driven by a net reduction in mortality during high pollution periods, further justifying our focus on the gradient analysis which leverages the fact that the mortality impact of the program is critically through its interaction with pollution. That is, rather than a (uniform) level shift in the mortality rate across different pollution levels, the program resulted in a change in the slope of the mortality-pollution relationship.

Staggered rollout In Panel (b) of Figure E.15, we compare the mortality rates of treated cities only with those of not-yet-treated cities. The results show that the estimated mortality impact is similar to the baseline when we compare wave one cities with waves two and three or wave three alone, and slightly larger when we compare wave two cities with wave three. This suggests that our main estimate of a 1.3-2.3 percentage point reduction in the mortalitypollution elasiticty (as reported in Table 3) may be conservative.

\section{Cost-Benefit Analysis of the Monitoring Program}

We end with a cost-benefit analysis of the monitoring program that quantifies the benefit and cost components outlined in Equation (B.2) in the appendix theory section. As this exercise depends on several assumptions and estimates that we borrow from the literature, we try to be conservative in the benefit estimation while more liberal in the cost estimation.

\subsection{Benefits of the Monitoring Program}

Mortality Benefit Together with our estimates on the change in mortality-pollution gradient in Section 6, the mortality benefit is quantified using age-adjusted value of statistical life (VSL). The VSL approach is commonly used by policymakers to evaluate the benefits of life-saving regulations. Due to the lack of reliable VSL estimates for the Chinese population, we use a benefit transfer method that infers the VSL for Chinese residents from U.S.-based VSL estimates and the income elasticity of VSL. The benefit-transfer method is commonly used for countries with insufficient or unreliable data (Viscusi and Masterman, 2017).

Ashenfelter and Greenstone (2004) estimate that the VSL in the U.S. is $\$ 2.3$ million, which can be considered as a lower bound. ${ }^{37}$ Narain and Sall (2016) suggest a transfer elasticity of 1.2 for transferring the VSL from U.S. to a developing country. Combined

\footnotetext{
${ }^{37}$ US EPA recommends a central VSL estimate of $\$ 7.4$ million in 2006 dollars, or $\$ 8.9$ million in 2015 dollars based on a meta analysis of VSL studies.
} 
with a one-to-nine income ratio between China and U.S., the VSL for population in China amounts to $¥ 1.3$ million in 2015. ${ }^{38}$ Based on the age adjustment suggested by Murphy and Topel (2006), the average age-adjusted VSL is estimated to be $¥ 0.49$ million for individuals 60 and above, among whom the mortality impact is concentrated. The $\beta$ estimate in Column (4) and Panel (D) in Table 4 suggest that the program has reduced total mortality for the age group 60 and above by at least 2.3 percentage points, amounting to a savings of 114,330 deaths per year. ${ }^{39}$ Therefore, the mortality benefit from reduced premature deaths among people 60 and above is $¥ 56.2$ billion (about $\$ 9$ billion) annually.

Morbidity Benefit The behavioral changes that reduce premature deaths could also lower morbidity. Relying on comprehensive data on health spending, recent studies such as Deschenes, Greenstone and Shapiro (2017) and Barwick et al. (2020) have shown that the healthcare cost alone, not accounting for the quality of life impact, is about two-thirds of the mortality cost in U.S. and China, respectively. This would imply a morbidity benefit of about ¥37.1 billion ( $\$ 5.9$ billion) per year.

The combined mortality and morbidity benefits amount to at least $¥ 93.3$ billion $(\$ 14.9$ billion) annually. This likely reflects a lower-bound of the program's benefit for several reasons. First, we use a conservative VSL estimate of $\$ 2.3$ million for the U.S. Second, the benefit calculation does not account for the quality-of-life changes or productivity gains from improved health. Third, to be consistent with the analysis on other outcomes, the mortality-pollution relationship is estimated on weekly data, and hence short-run in nature. In an analysis based on quarterly data, we find the longer-run reduction in the mortalitypollution gradient is indeed slightly larger (more lives saved than reported in Table 4.

\subsection{Costs of the Program}

We now turn to the costs of avoidance, which include increased defensive spending, the welfare loss from foregone consumption, and the cost of the monitoring program itself. First, we consider purchases of air purifiers and masks as defensive expenditures. Aggregate annual sales of air purifiers and facial masks grew from ¥4 billion in 2012 to $¥ 13.5$ billion in 2015 . The average increase in annual sales amount to ¥7.5 billion during 2013-2015 relative to 2012. ${ }^{40}$ One caveat in this calculation is that a complete cost calculation of defensive spending

\footnotetext{
${ }^{38} \mathrm{VSL}$ for China $=\$ 2.3 /\left(1.2^{*} 9\right)$ mill $=\$ 0.21$ mill, or $¥ 1.3$ mill.

${ }^{39}$ The total number of deaths among people 60 and above is 4.971 million in 2012 according to China Statistical Yearbook.

${ }^{40}$ For air purifier sales, see "Industry Trends for China's Air Purifiers" by Growth for Knowledge (2015); for facial mask sales, see " $\mathrm{PM}_{2.5}$ Facial Masks Industry Report" by Intelligence Research Group (2017).
} 
would necessitate information on drug uses, changes in residential locations, as well as other non-market activities (such as outdoor exercises), which we do not consider in this paper.

Second, to calculate the welfare loss from foregone consumption, we use estimates from Table 2 to calculate foregone consumption and assume the price elasticity for consumer goods to vary from -1 to -1.5 (Deaton and Muellbauer, 1980; Blundell, Pashardes and Weber, 1993; Banks, Blundell and Lewbel, 1997). The estimated deadweight loss from the foregone consumption ranges between $¥ 1.3$ and 1.9 billion. ${ }^{41}$ The estimated foregone consumption are upper bounds as some of them are likely deferred rather than permanently foregone: as shown in Section 5.1, the effect on bank-card use appears to concentrate on "deferrable" categories, many of which are temporally substitutable in nature.

Third, the one-time cost to set up the monitoring stations and broadcast the pollution information online is estimated to be at most $¥ 2$ billion, and the annual operation and maintenance cost is at most $¥ 0.1$ billion a year. ${ }^{42}$ Taken together, a likely upper bound of the total costs is $¥ 11.5$ billion in its first year (including the set-up costs) and $¥ 9.5$ billion annually thereafter.

The estimated annual benefits and costs of the program - at least $¥ 93.3$ billion of health benefits relative to the associated costs of $¥ 9.5$ billion - underscores the cost-effectiveness of the information monitoring and disclosure program. ${ }^{43}$ Empowering the public with real-time pollution information mobilizes individuals' ability to mitigate the adverse consequences of pollution at a relatively low cost. This, together with other environmental regulations, can more effectively reduce the societal cost of pollution.

\section{Conclusions}

This paper examines the role of pollution information in enhancing households' awareness of pollution problems, promoting pollution avoidance behaviors, and mitigating pollution's

\footnotetext{
${ }^{41}$ The deadweight loss can be approximated by $\frac{1}{2} \times \Delta$ consumption $\times \frac{\tau}{|\epsilon|}$, where $\Delta$ consumption is the change annual in consumption (in $¥$ ) due to the program; $\tau$ is the ratio of the change in consumption over total consumption; and $\epsilon$ is the price elasticity for consumption. The $\beta$ estimate in Column (4) and Panel A in Table 2 suggests that $\tau$ is 1.4 percent (12.17 transactions out of 869 per 10,000 cards), and that $\Delta$ consumption amounts to $¥ 278$ billion.

${ }^{42}$ Chinese central and local governments invested $¥ 1.8$ billion in setting up the nationwide monitoring system (Wang, 2014). The winning bids for the operation and maintenance of all the monitors amount to about ¥213 million from 2016 to 2018 according to the announcement from the Chinese government procurement information platform.

${ }^{43}$ Successful environmental regulations such as the U.S. Acid Rain Program and the Clean Air Act have been shown to achieve benefits an order of magnitude larger than the cost (Krupnick and Morgenstern, 2002; Chestnut and Mills, 2005). Different from our context, these programs substantially reduced pollution levels.
} 
negative health impacts. We focus on a landmark policy change in China that resulted in the installation of air pollution monitoring stations nationwide and the provision to the public of the real-time pollution information that previously had been unavailable. Based on several rich and unique data sets, our analysis provides strong evidence that the program resulted in cascading changes such as increased access to pollution information, enhanced awareness of pollution's harm, more pronounced avoidance behavior, and higher defensive spending. The risk-compensating behavioral changes ultimately led to a reduction in the negative health effects of air pollution. Our estimates suggest that the health benefits of the information monitoring and disclosure program is nearly one order of magnitude larger than the combined costs of the program implementation itself and association behavioral changes.

China's experience offers an important lesson for other developing countries that are facing severe environmental challenges. In many low-income countries the infrastructure for monitoring environmental quality and disclosing the information is often inadequate or does not exist. As income rises, the demand for environmental quality increases, and households are better able to adapt to adverse environmental conditions. Providing real-time pollution data - combined with effective dissemination infrastructure such as smartphones and the Internet, both of which are increasingly available in developing countries - could be a powerful and cost-effective tool to help households mitigate health damages from environmental pollution. Such information transparency and distribution may also enhance the effectiveness of other environmental regulations and related enforcement mechanisms (Greenstone et al., 2022) that are designed to reduce air pollution. Furthermore, the optimal design of the monitoring system - a subject that in itself warrants more research attention - should help provide more accurate air quality measurements and thus help unleash the full extent of health benefits that can accrue through the provision of accurate, actionable information.

Finally, while the finding of large health benefits from air quality monitoring and disclosure program is consistent with the notion that information remedies can serve as a quasi-regulatory mechanism (Konar and Cohen, 1997), our findings do not suggest that such information programs can or should serve as a substitute for policies that aim to reduce the dangerous levels of air pollution in many developing countries. The dramatic improvement in air quality in China during the last few years likely would not have occurred without a series of dramatic and stringent policies to reduce emissions (Greenstone et al., 2021; Karplus, Zhang and Zhao, 2021). By facilitating better enforcement and civic engagement, the monitoring program might have enhanced the effectiveness of environmental regulations to improve air quality. We consider this an important question for future research. 


\section{References}

Abadie, Alberto, Alexis Diamond, and Jens Hainmueller. 2010. "Synthetic control methods for comparative case studies: Estimating the effect of California's tobacco control program." Journal of the American statistical Association, 105(490): 493-505.

Agarwal, Sumit, Yu Qin, and Hongjia Zhu. 2020. "Disguised pollution: Industrial activities in the dark." Available at SSRN 3359404.

Aguilar-Gomez, Sandra, Holt Dwyer, Joshua S Graff Zivin, and Matthew J Neidell. 2022. "This is Air: The 'Non-Health' Effects of Air Pollution." National Bureau of Economic Research Working Paper 29848.

Allcott, Hunt. 2013. "The Welfare Effects of Misperceived Product Costs: Data and Calibrations from the Automobile Market." American Economic Journal: Economic Policy, 5(3): 30-66.

Almond, Douglas, Hongbin Li, and Shuang Zhang. 2019. "Land reform and sex selection in China." Journal of Political Economy, 127(2): 560-585.

Anderson, Michael L. 2020. "As the Wind Blows: The Effects of Long-Term Exposure to Air Pollution on Mortality." Journal of the European Economic Association, 18(4): 18861927.

Andrews, Steven. 2008. "Inconsistencies in air quality metrics: 'Blue Sky' days and PM10 concentrations in Beijing." Environmental Research Letters, 034009(3).

Arceo, Eva, Rema Hanna, and Paulina Oliva. 2015. "Does the Effect of Pollution on Infant Mortality Differ Between Developing and Developed Countries? Evidence from Mexico City." Economic Journal, 126: 257-280.

Arkhangelsky, Dmitry, Susan Athey, David A Hirshberg, Guido W Imbens, and Stefan Wager. 2021. "Synthetic difference-in-differences." American Economic Review, 111(12): 4088-4118.

Ashenfelter, Orley, and Michael Greenstone. 2004. "Estimating the Value of a Statistical Life: The Importance of Omitted Variables and Publication Bias." American Economic Review, 94(2): 454-460.

Bai, Jie. 2018. "Melons as Lemons: Asymmetric Information, Consumer Learning and Quality Provision." Working Paper.

Banks, James, Richard Blundell, and Arthur Lewbel. 1997. "Quadratic Engel Curves and Consumer Demand." The Review of Economics and Statistics, 79(4): 527-539.

Banzhaf, H. Spencer, and Randall P. Walsh. 2008. "Do People Vote with Their Feet? An Empirical Test of Tiebout." American Economic Review, 98: 843-863.

Barwick, Panle Jia, Shanjun Li, Deyu Rao, and Nahim Bin Zahur. 2020. "The Morbidity Cost of Air Pollution: Evidence from the World's Largest Payment Network." NBER Working Paper. 
Barwick, Panle Jia, Shanjun Li, Liguo Lin, and Eric Zou. 2019. "From fog to smog: The value of pollution information." National Bureau of Economic Research.

Bayer, Patrick, Nate Keohane, and Christopher Timmins. 2009. "Migration and Hedonic Valuation: The Case of Air Quality." Journal of Environmental Economics and Management, 58: 1-14.

Bernheim, B. Douglas, and Antonio Rangel. 2009. "Beyond Revealed Preference: Choice-Theoretic Foundations for Behavioral Welfare Economics." The Quarterly Journal of Economics, 124(1): 51-104.

Bernheim, B. Douglas, Andrey Fradkin, and Igor Popov. 2015. "The Welfare Economics of Default Options in 401(k) Plans." American Economic Review, 105(9): 27982837.

Blundell, Richard, Panos Pashardes, and Guglielmo Weber. 1993. "What do we Learn About Consumer Demand Patterns from Micro Data?" The American Economic Review, 83(3): 570-597.

Bollinger, Bryan, Phillip Leslie, and Alan Sorensen. 2011. "Calorie posting in chain restaurants." American Economic Journal: Economic Policy, 3(1): 91-128.

Callaway, Brantly, and Pedro HC Sant'Anna. 2020. "Difference-in-differences with multiple time periods." Journal of Econometrics.

Chang, Tom Y., Joshua Graff Zivin, Tal Gross, and Matthew Neidell. 2019. "The Effect of Pollution on Worker Productivity: Evidence from Call Center Workers in China." American Economic Journal: Applied Economics, 11(1): 151-72.

Chay, Kenneth Y., and Michael Greenstone. 2005. "Does air quality matter? Evidence from the housing market." Journal of Political Economy, 113(2): 376-424.

Chen, Shuai, Paulina Oliva, and Peng Zhang. 2017. "The Effect of Air Pollution on Migration: Evidence from China." NBER Working Paper No. 24036.

Chen, Yuyu, Ginger Jin, Naresh Kumar, and Guang Shi. 2012. "Gaming in Air Pollution Data? Lessons from China." B.E. Journal of Economic Analysis 86 Policy, 3(12): e313 - e323.

Chestnut, Lauraine G., and David M. Mills. 2005. "A fresh look at the benefits and costs of the US acid rain program." Journal of Environmental Management, 77(3): 252 266.

Chetty, Raj, Adam Looney, and Kory Kroft. 2009. "Salience and Taxation: Theory and Evidence." American Economic Review, 99(4): 1145-77.

Craft, Erik D. 1998. "The Value of Weather Information Services for Nineteenth-Century Great Lakes Shipping." American Economic Review, 88(5): 1059-76.

Cutter, W. Bowman, and Matthew Neidell. 2009. "Voluntary information programs and environmental regulation: Evidence from 'Spare the Air'." Journal of Environmental 
Economics and Management, 58(3): 253 - 265.

Dasgupta, Susmita, Benoit Laplante, Hua Wang, and David Wheeler. 2002. "Confronting the Environmental Kuznets Curve." Journal of Economic Perspectives, 16(1): 147168.

Deaton, Angus, and John Muellbauer. 1980. "An Almost Ideal Demand System." The American Economic Review, 70(3): 312-326.

De Chaisemartin, Clément, and Xavier d'Haultfoeuille. 2020a. "Difference-indifferences estimators of intertemporal treatment effects." Available at SSRN 3731856.

De Chaisemartin, Clément, and Xavier d'Haultfoeuille. 2020b. "Two-way fixed effects estimators with heterogeneous treatment effects." American Economic Review, 110(9): 2964-96.

Deryugina, Tatyana, Garth Heutel, Nolan H. Miller, David Molitor, and Julian Reif. 2019. "The Mortality and Medical Costs of Air Pollution: Evidence from Changes in Wind Direction." American Economic Review, 109(12): 4178-4219.

Deschenes, Olivier, and Michael Greenstone. 2011. "Climate Change, Mortality, and Adaptation: Evidence from Annual Fluctuations in Weather in the US." American Economic Journal: Applied Economics, 3(4): 152-185.

Deschenes, Olivier, Michael Greenstone, and Joseph Shapiro. 2017. "Defensive Investments and the Demand for Air Quality: Evidence from the NOx Budget Program." American Economic Review, 107(10): 2958-89.

Dranove, David, and Ginger Zhe Jin. 2010. "Quality Disclosure and Certification: Theory and Practice." Journal of Economic Literature, 48(4): 935-63.

Duflo, Esther, and Emmanuel Saez. 2003. "The Role of Information and Social Interactions in Retirement Plan Decisions: Evidence from a Randomized Experiment." The Quarterly Journal of Economics, 118(3): 815-842.

Duflo, Esther, Michael Greenstone, Rohini Pande, and Nicholas Ryan. 2018. "The Value of Regulatory Discretion: Estimates From Environmental Inspections in India." Econometrica, 86(6): 2123-2160.

Ebenstein, Avraham, Maoyong Fan, Michael Greenstone, Guojun He, and Maigeng Zhou. 2017. "New evidence on the impact of sustained exposure to air pollution on life expectancy from China's Huai River Policy." Proceedings of the National Academy of Sciences, 114: 10384-10389.

Fedorenko, Irina, and Yixian Sun. 2016. "Microblogging-Based Civic Participation on Environment in China: A Case Study of the PM 2.5 Campaign." VOLUNTAS, 27: 20772105.

Fowlie, Meredith, Edward Rubin, and Reed Walker. 2019. "Bringing satellite-based air quality estimates down to earth." Vol. 109, 283-88. 
Gao, Xuwen, Ran Song, and Christopher Timmins. 2021. "The Role of Information in the Rosen-Roback Framework." National Bureau of Economic Research Working Paper 28943.

Ghanem, Dalia, and Junjie Zhang. 2014. "Effortless Perfection:' Do Chinese cities manipulate air pollution data?" Journal of Environmental Economics and Management, 68(2): $203-225$.

Goodman-Bacon, Andrew. 2021. "Difference-in-differences with variation in treatment timing." Journal of Econometrics.

Graff Zivin, Joshua, and Matthew Neidell. 2009. "Days of haze: Environmental information disclosure and intertemporal avoidance behavior." Journal of Environmental Economics and Management, 58(2): 119-128.

Graff Zivin, Joshua, and Matthew Neidell. 2012. "The Impact of Pollution on Worker Productivity." American Economic Review, 102(7): 3652-73.

Greenstone, Michael, and B. Kelsey Jack. 2015. "Envirodevonomics: A Research Agenda for an Emerging Field." Journal of Economic Literature, 53(1): 5-42.

Greenstone, Michael, and Patrick Schwarz. 2018. "Is China Winning its War on Pollution?" Report from Energy Policy Institute at the University of Chicago.

Greenstone, Michael, and Rema Hanna. 2014. "Environmental Regulations, Air and Water Pollution, and Infant Mortality in India." American Economic Review, 104: 30383072 .

Greenstone, Michael, Guojun He, Ruixue Jia, and Tong Liu. 2022. "Can Technology Solve the Principal-Agent Problem? Evidence from Pollution Monitoring in China." American Economic Review: Insights.

Greenstone, Michael, Guojun He, Shanjun Li, and Eric Yongchen Zou. 2021. "China's War on Pollution: Evidence from the First Five Years." Review of Environmental Economics and Policy, , (2): 281-299.

Grossman, Michael. 1972. "On the Concept of Health Capital and Demand for Health." The Journal of Political Economy, 80: 223-255.

Hanna, Rema, and Paulina Oliva. 2015. "The effect of pollution on labor supply: Evidence from a natural experiment in Mexico City." Journal of Public Economics, 122: 68 79 .

Hao, Jiming, and Litao Wang. 2005. "Improving Urban Air Quality in China: Beijing Case Study." Journal of the Air \&6 Waste Management Association, 55(9): 1298-1305.

Harrington, Winston, and Paul R. Portney. 1987. "Valuing the benefits of health and safety regulation." Journal of Urban Economics, 22(1): 101-112.

Hastings, Justine S., and Jeffrey M. Weinstein. 2008. "Information, School Choice, and Academic Achievement: Evidence from Two Experiments*." The Quarterly Journal 
of Economics, 123(4): 1373-1414.

He, Jiaxiu, Haoming Liu, and Alberto Salvo. 2019. "Severe Air Pollution and Labor Productivity: Evidence from Industrial Towns in China." American Economic Journal: Applied Economics, 11(1): 173-201.

Hilton, Ronald W. 1981. "The Determinants of Information Value: Synthesizing Some General Results." Management Science, 27(1): 57-64.

Hirshleifer, Jack. 1971. "The Private and Social Value of Information and the Reward to Inventive Activity." The American Economic Review, 61(4): 561-574.

Huang, Jing, Xiaochuan Pan, Xinbiao Guo, and Guoxing Li. 2018. "Health impact of China's Air Pollution Prevention and Control Action Plan: an analysis of national air quality monitoring and mortality data." The Lancet Planetary Health, 2(7): e313 - e323.

Ito, Koichiro. 2014. "Do Consumers Respond to Marginal or Average Price? Evidence from Nonlinear Electricity Pricing." American Economic Review, 104(2): 537-63.

Ito, Koichiro, and Shuang Zhang. 2018. "Willingness to Pay for Clean Air: Evidence from Air Purifier Markets in China." Journal of Political Economy. forthcoming.

Jagnani, Maulik, Christopher Barrett, Yanyan Liu, and Liangzhi You. 2018. "Within-Season Producer Response to Warmer Temperatures: Defensive Investments by Kenyan Farmers." Working Paper.

Jessoe, Katrina, and David Rapson. 2014. "Knowledge Is (Less) Power: Experimental Evidence from Residential Energy Use." American Economic Review, 104(4): 1417-38.

Jha, Akshaya, and Andrea La Nauze. 2022. "US Embassy air-quality tweets led to global health benefits." Proceedings of the National Academy of Sciences, 119(44): e2201092119.

Jiang, Bowen, Yuangang Li, and Weixin Yang. 2020. "Evaluation and Treatment Analysis of Air Quality Including Particulate Pollutants: A Case Study of Shandong Province, China." nternational journal of environmental research and public health, , (24): 9476.

Jin, Ginger Zhe, and Phillip Leslie. 2003. "The Effect of Information on Product Quality: Evidence from Restaurant Hygiene Grade Cards." The Quarterly Journal of Economics, 118(2): 409-451.

Jones, Damon, David Molitor, and Julian Reif. 2019. "What do Workplace Wellness Programs do? Evidence from the Illinois Workplace Wellness Study*." The Quarterly Journal of Economics, 134(4): 1747-1791.

Kahn, Matthew E., and Siqi Zheng. 2016. Blue Skies over Beijing: Economic Growth and the Environment in China. Princeton University Press.

Karplus, Valerie J., Junjie Zhang, and Jinhua Zhao. 2021. "Navigating and Evaluating the Labyrinth of Environmental Regulation in China." Review of Environmental Economics and Policy, 15(2): 300-322. 
Karplus, Valerie J., Shuang Zhang, and Douglas Almond. 2018. "Quantifying coal power plant responses to tighter SO2 emissions standards in China." Proceedings of the National Academy of Sciences, 115(27): 7004-7009.

Kling, Jeffrey R., Sendhil Mullainathan, Eldar Shafir, Lee C. Vermeulen, and Marian V. Wrobel. 2012. " Comparison Friction: Experimental Evidence from Medicare Drug Plans." The Quarterly Journal of Economics, 127(1): 199-235.

Konar, Shameek, and Mark A. Cohen. 1997. "Information As Regulation: The Effect of Community Right to Know Laws on Toxic Emissions." Journal of Environmental Economics and Management, 32(1): $109-124$.

Krupnick, Alan, and Richard Morgenstern. 2002. "The Future of Benefit-Cost Analyses of the Clean Air Act." Annual Review of Public Health, 23(1): 427-448.

Landrigan, Philip, Richard Fuller, Nereus J R Acosta, Olusoji Adeyi, Maureen Cropper, Alan Krupnick, Michael Greenstone, and et al. 2018. "The Lancet Commission on pollution and health." The Lancet, 391(10119): 462-512.

Lave, Lester B. 1963. "The Value of Better Weather Information to the Raisin Industry." Econometrica, 31(1/2): 151-164.

Mastromonaco, Ralph. 2015. "Do environmental right-to-know laws affect markets? Capitalization of information in the toxic release inventory." Journal of Environmental Economics and Management, 71: $54-70$.

Murphy, Kevin M., and Robert H. Topel. 2006. "The Value of Health and Longevity." Journal of Political Economy, 114(5): 871-904.

Narain, Urvashi, and Chris Sall. 2016. Methodology for Valuing the Health Impacts of Air Pollution: Discussion of Challenges and Proposed Solutions. The World Bank.

Neidell, Matthew. 2009. "Information, Avoidance Behavior, and Health: The Effect of Ozone on Asthma Hospitalizations." Journal of Human Resources, 44(2): 1209-1236.

Newell, Richard G., and Juha Siikamäki. 2014. "Nudging Energy Efficiency Behavior: The Role of Information Labels." Journal of the Association of Environmental and Resource Economists, 1(4): 555-598.

Oberholzer-Gee, Felix, and Miki Mitsunari. 2006. "Information regulation: Do the victims of externalities pay attention?" Journal of Regulatory Economics, 30(2): 141-158.

Pope, C. Arden, and Douglas W. Dockery. 2012. "Health Effects of Fine Particular Air Pollution: Lines that Connect." Journal of the Air and Waste Management Association, 56: 709-742.

Qian, Nancy, and Jaya Wen. 2015. "The Impact of Xi Jinping's Anti-Corruption Campaign on Luxury Imports in China." Northwestern Working Paper.

Saberian, Soodeh, Anthony Heyes, and Nicholas Rivers. 2017. "Alerts work! Air quality warnings and cycling." Resource and Energy Economics, 49: 165 - 185. 
Shin, Jeong-Shik. 1985. "Perception of Price When Price Information Is Costly: Evidence from Residential Electricity Demand." The Review of Economics and Statistics, 67(4): 591598.

Shrader, Jeffrey. 2018. "Expectations and adaptation to environmental risks." Working Paper.

Smith, V. Kerry, and F. Reed Johnson. 1988. "How do Risk Perceptions Respond to Information? The Case of Radon." The Review of Economics and Statistics, 70(1): 1-8.

Stigler, George J. 1961. "The economics of information." Journal of Political Economy, 69(3): 213-225.

Stigler, George J. 1962. "Information in the Labor Market." Journal of Political Economy, 70(5): 94-105.

Sun, Liyang, and Sarah Abraham. 2020. "Estimating dynamic treatment effects in event studies with heterogeneous treatment effects." Journal of Econometrics.

Tanaka, Shinsuke. 2015. "Environmental regulations on air pollution in China and their impact on infant mortality." Journal of Health Economics, 42: 90 - 103.

Tu, Meng, Bing Zhang, Jianhua Xu, and Fangwen Lu. 2020. "Mass media, information and demand for environmental quality: Evidence from the "Under the Dome"." Journal of Development Economics, 143: 102402.

Van Donkelaar, Aaron, Randall V Martin, and Rokjin J Park. 2006. "Estimating ground-level PM2. 5 using aerosol optical depth determined from satellite remote sensing." Journal of Geophysical Research: Atmospheres, 111(D21).

Van Donkelaar, Aaron, Randall V Martin, Michael Brauer, N Christina Hsu, Ralph A Kahn, Robert C Levy, Alexei Lyapustin, Andrew M Sayer, and David M Winker. 2016. "Global estimates of fine particulate matter using a combined geophysical-statistical method with information from satellites, models, and monitors." Environmental science 60 technology, 50(7): 3762-3772.

Viscusi, W. Kip, and Clayton J. Masterman. 2017. "Income Elasticities and Global Values of a Statistical Life." Journal of Benefit-Cost Analysis, 8(2): 226-250.

Wainwright, Oliver. 2014. "Inside Beijing's airpocalypse - a city made 'almost uninhabitable' by pollution." The Guardian.

Wang, Alex L. 2017. "Explaining environmental information disclosure in China." Ecology Law Quarterly, 44: 865.

Wang, Erde. 2014. "Nationwide PM2.5 Monitoring Coverage from 2015." Century Business Herald.

Westfall, Peter, and Stanley Young. 1993. Resampling-based Multiple Testing: Examples and Methods for p-value Adjustment. John Wiley \& Sons. 
Wichman, Casey J. 2017. "Information provision and consumer behavior: A natural experiment in billing frequency." Journal of Public Economics, 152: 13 - 33.

Williams, Austin M, and Daniel J Phaneuf. 2019. "The morbidity costs of air pollution: Evidence from spending on chronic respiratory conditions." Environmental and Resource Economics, 74(2): 571-603.

Yi, Honghong, Jiming Hao, and Xiaolong Tang. 2007. "Atmospheric environmental protection in China: Current status, developmental trend and research emphasis." Energy Policy, 35(2): 907 - 915.

Zhang, Junjie, and Quan Mu. 2018. "Air pollution and defensive expenditures: Evidence from particulate-filtering facemasks." Journal of Environmental Economics and Management, 92: 517 - 536 .

Zhang, Junjie, Zhenxuan Wang, and Xinming Du. 2017. "Lessons learned from China's regional carbon market pilots." Economics of Energy \&f Environmental Policy, 0 (Number 2).

Zhou, Maigeng, Haidong Wang, Jun Zhu, Wanqing Chen, Linhong Wang, Shiwei Liu, Yichong Li, Lijun Wang, Yunning Liu, Peng Yin, et al. 2016. "Cause-specific mortality for 240 causes in China during 1990-2013: a systematic subnational analysis for the Global Burden of Disease Study 2013." The Lancet, 387(10015): 251-272.

Zivin, Joshua Graff, and Matthew Neidell. 2009. "Days of haze: Environmental information disclosure and intertemporal avoidance behavior." Journal of Environmental Economics and Management, 58(2): 119 - 128. 
Figure 1: Confusion between Heavy Smog and Dense Fog by Government Agencies

(a) News coverage on government website

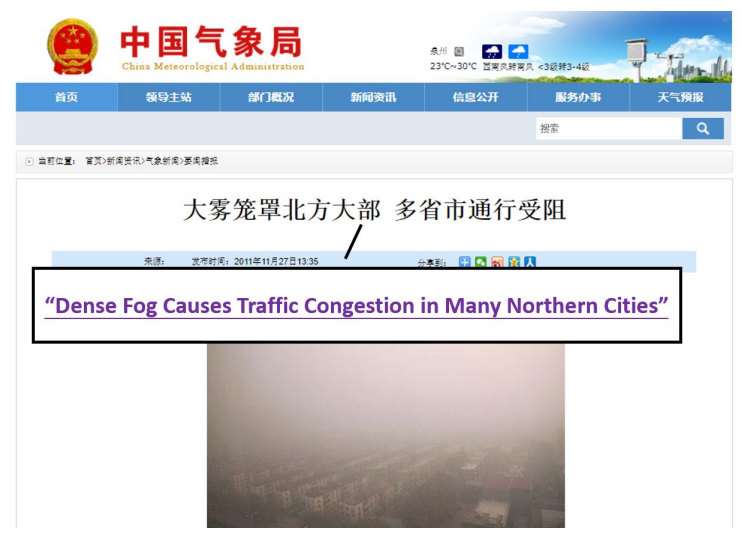

(b) Satellite picture of the event

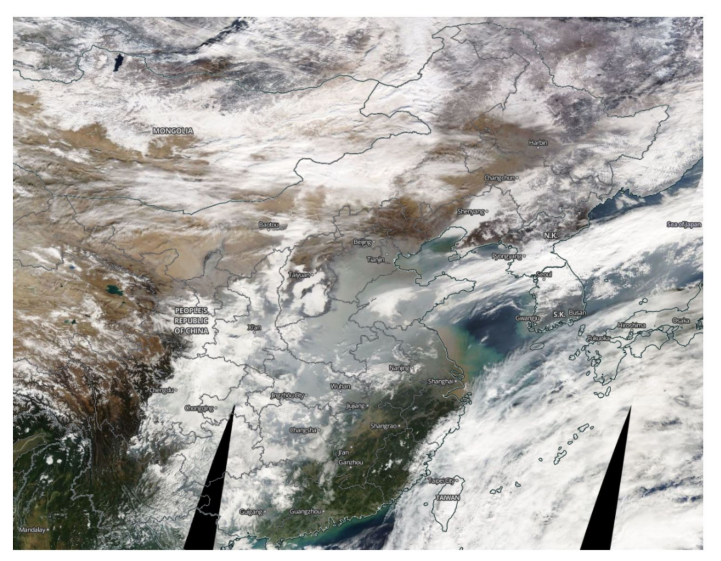

(c) Satellite-derived pollution levels

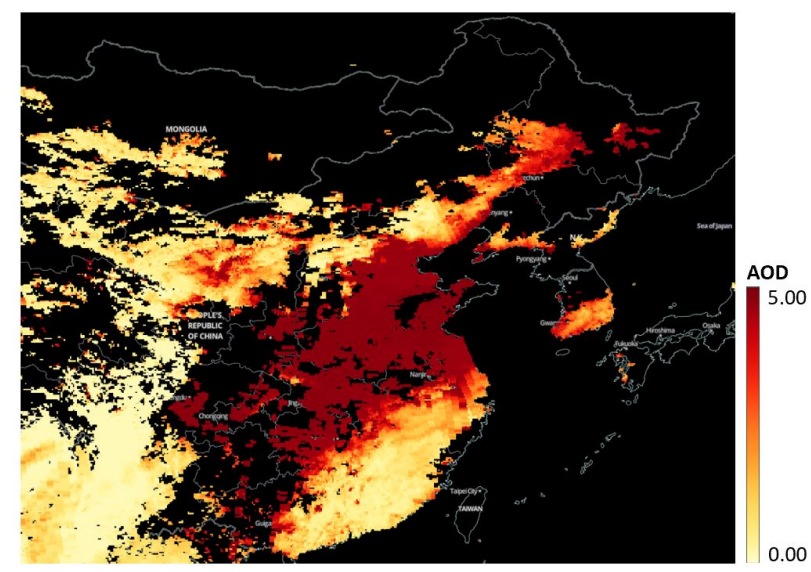

Notes: This figure illustrates a "widespread, dense fog event" on November 27, 2011 which is in fact a major pollution event. Panel (a), sourced from China Meteorological Administration, shows official news coverage of the event. Panel (b), sourced from NASA, shows the satellite view of China on the same day. Panel (c), sourced from NASA MODIS algorithm, shows the satellite-based measure of pollution (aerosol optical depth). Dark red regions have high levels of pollution. Black regions have missing data (with cloud cover or water surfaces). 
Figure 2: Monitoring Program Rollout and Mortality Data Availability

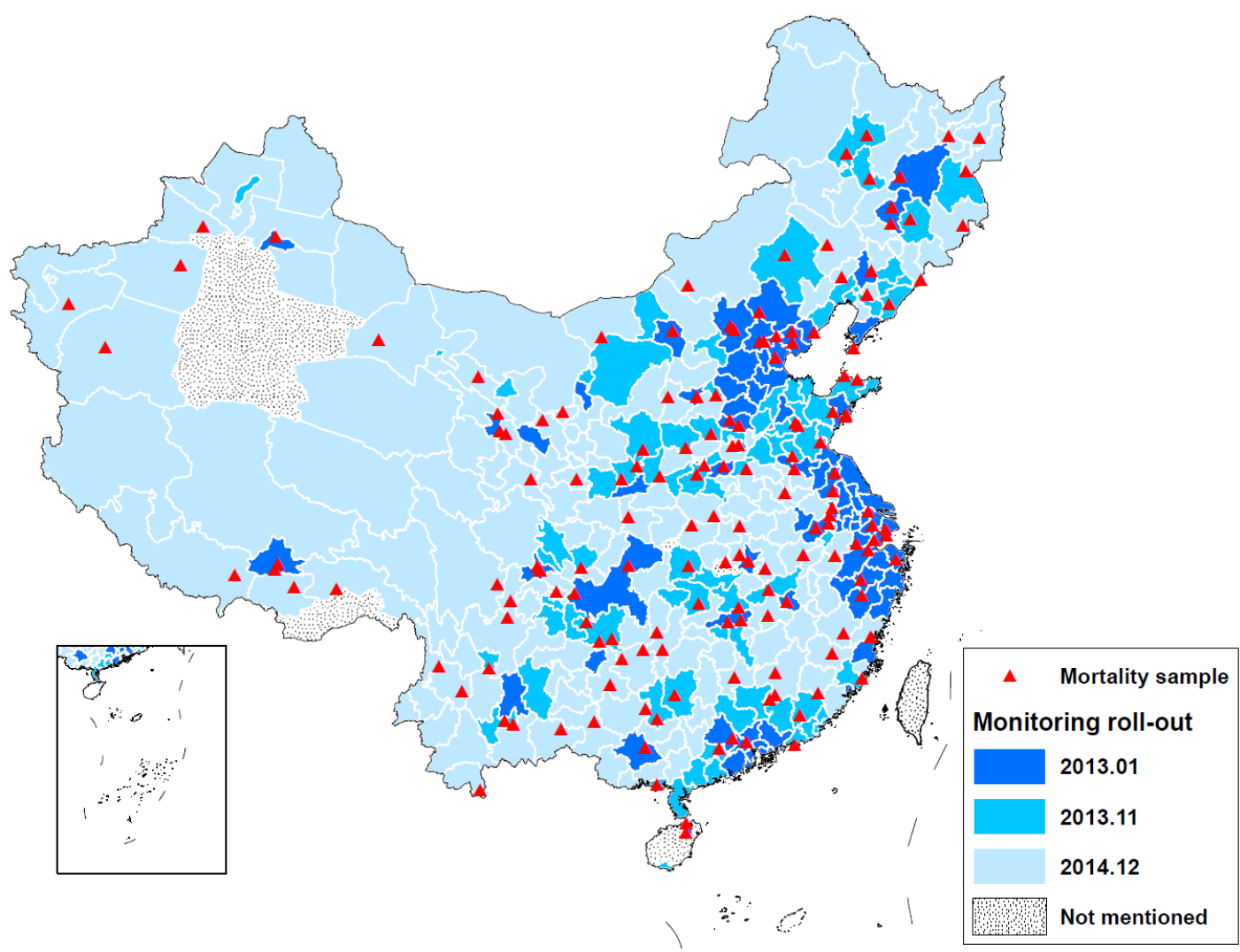

Notes: This map shows the list of cities by the completion date of the air pollution monitoring and disclosure program. "Not mentioned" are cities where the timing of monitoring is not mentioned in the government's policy. "Mortality sample" are cities included in the DSP mortality data. 


\section{Figure 3: Changes in Pollution Information Access - News Mentions}

(a) "Smog" news in People's Daily

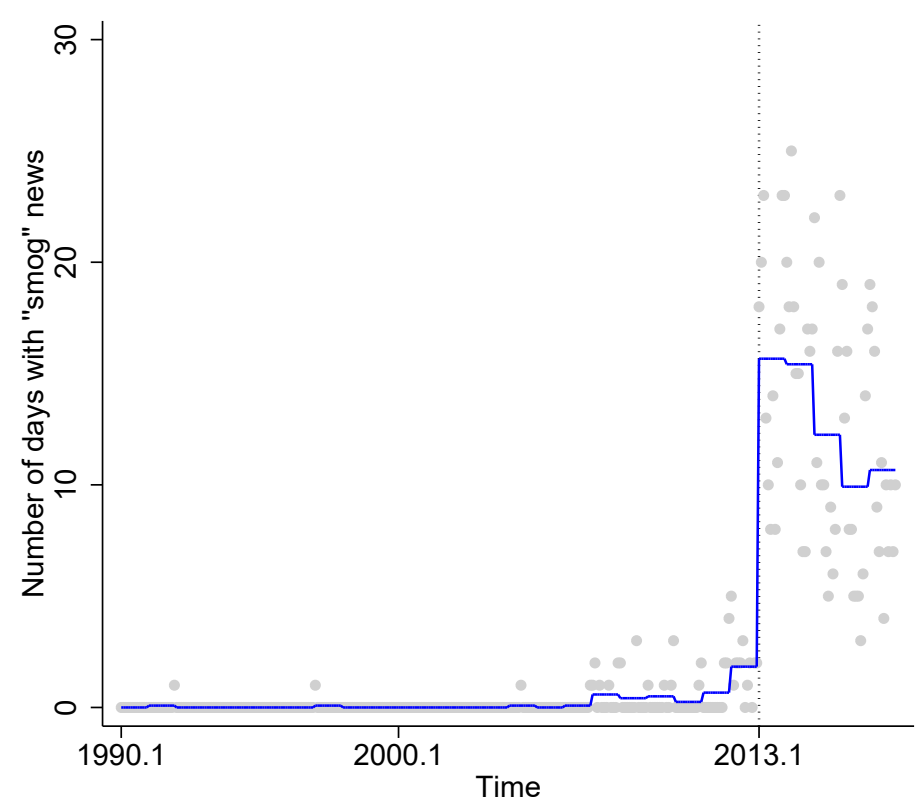

(b) Local "smog" news before and after monitoring

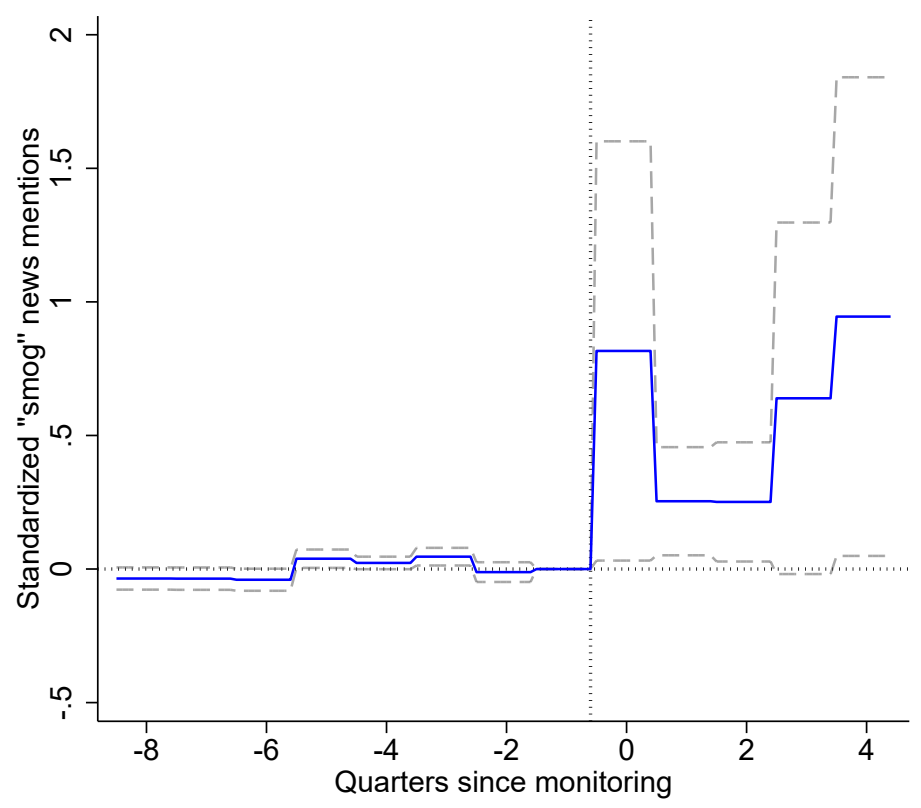

Notes: Panel (a) plots the number of days in each month when the People's Daily, the official newspaper of the Chinese government which reports both national and local news, published articles containing "smog" in content. Each dot represents a month. Line shows annual averages. Panel (b) plots standardized city-level "smog" mentions, defined as news that mentions both "smog" and the city name, as a function of quarters since the completion of the monitoring program in a given city. Event quarter -1 is normalized to 0 . The underlying regression contains no control variables; see Appendix Figure E. 6 for versions with fixed effects controls. Dashed lines show 95\% confidence interval constructed from standard errors clustered at the city level. 
Figure 4: Changes in Pollution Awareness - Baidu Smog Search Index

(a) Baidu "smog" search index at the national level

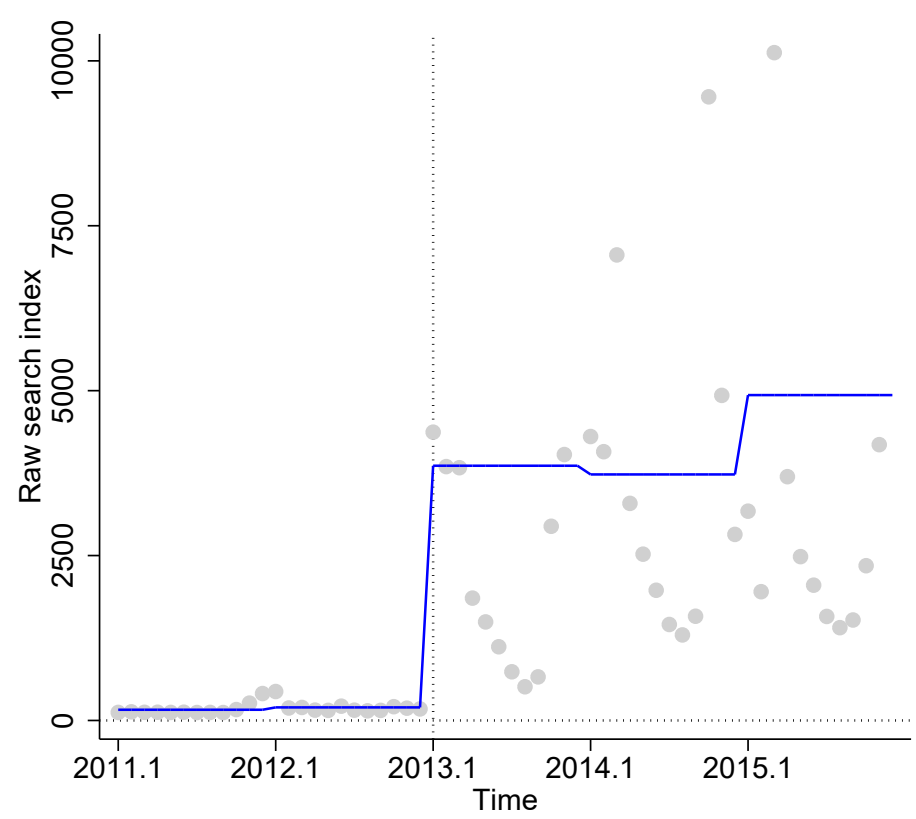

(b) Local Baidu "smog" search index by Event Time

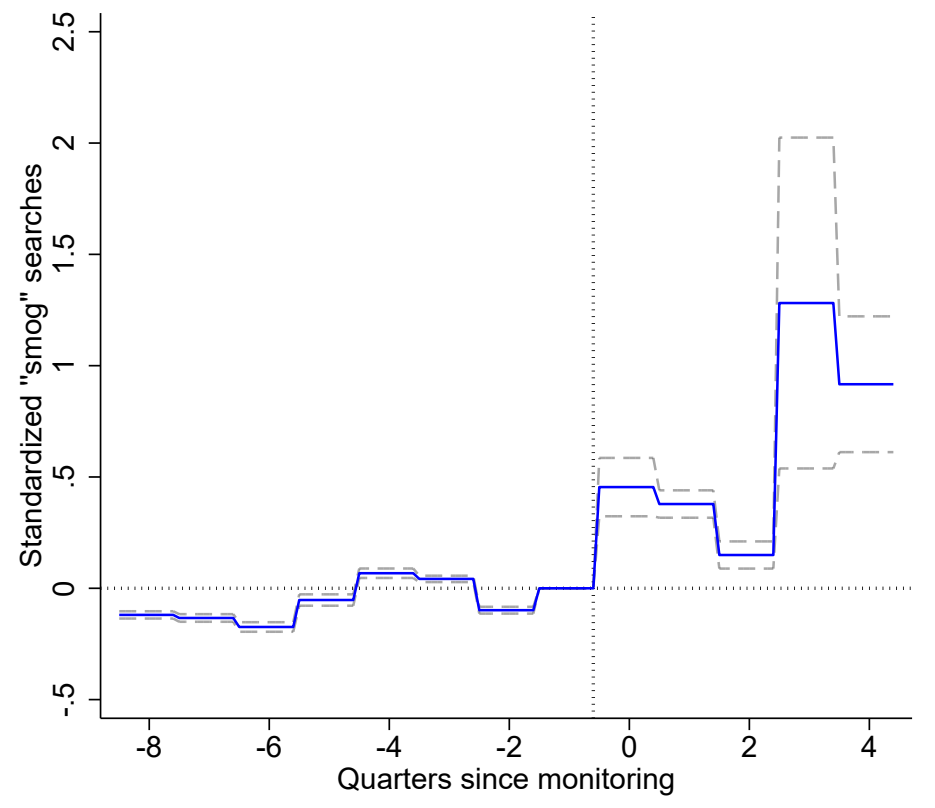

Notes: Panel (a) plots raw monthly trends in Baidu Search Index for keyword "smog". The graph omits two dots with exceptionally high search index (December 2013 with index $=20,942$ and December 2015 with index $=24,679$ ) for readability. Line shows annual averages. Panel (b) plots standardized "smog" search index as a function of quarters since the completion of the monitoring program in a given city. Event quarter -1 is normalized to 0 . The underlying regression contains no control variables; see Appendix Figure E.6 for versions with fixed effects controls. Dashed lines show 95\% confidence interval constructed from standard errors clustered at the city level. 
Figure 5: Changes in Public Attitudes toward Environmental, Economics, and Social Issues

(a) Changes in expressed level of concerns after the monitoring program

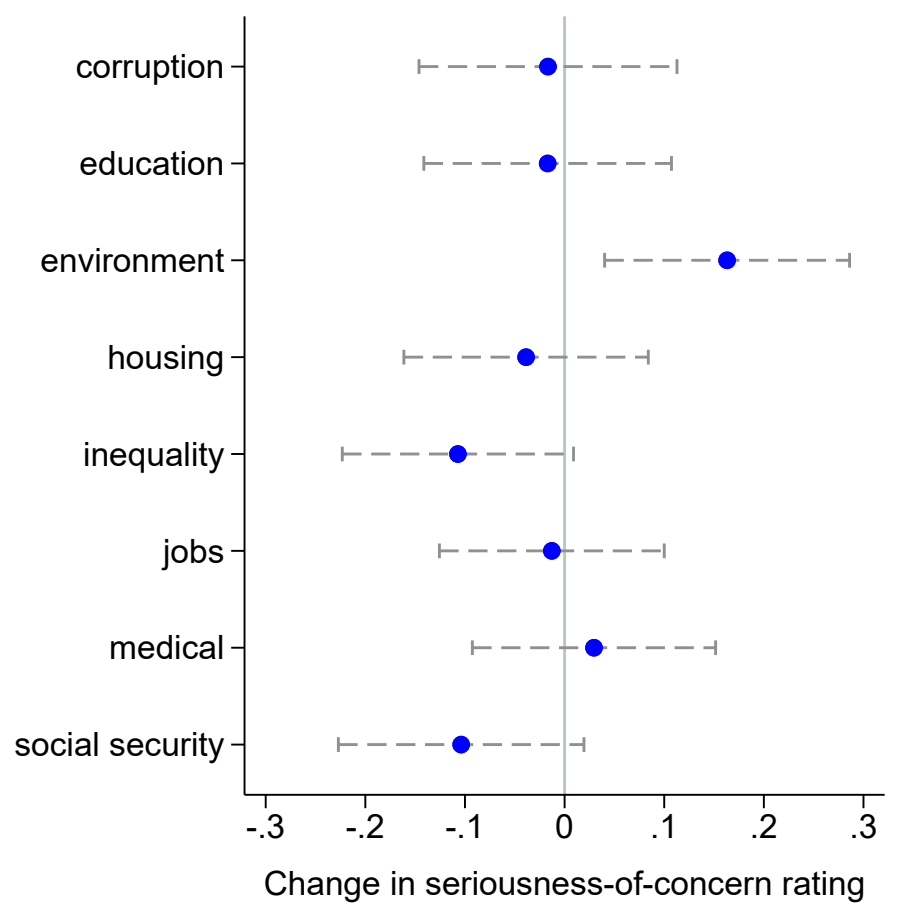

(b) Environmental concerns by local pollution level

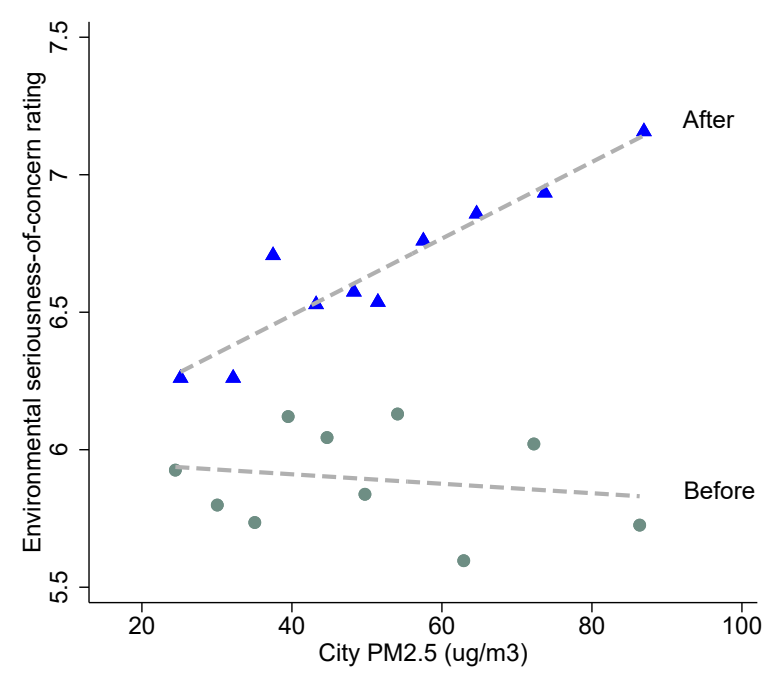

Notes: Panel (a) examines whether the monitoring program changed the public's attitudes towards various subjects, regressing respondents' level-of-concern metric on a post-monitoring dummy, controlling for respondent fixed effects and year fixed effects. Each bar corresponds to a separate regression, showing the coefficient estimate on the post-monitoring dummy and the $95 \%$ confidence interval. Panel (b) displays bin scatterplots between respondents' level-of-concern on environmental issues and their local city's 2015-2016 average $\mathrm{PM}_{2.5}$ concentration, separately by before and after the monitoring program. 
Figure 6: Changes in Avoidance Behavior - Shopping-Pollution Gradient

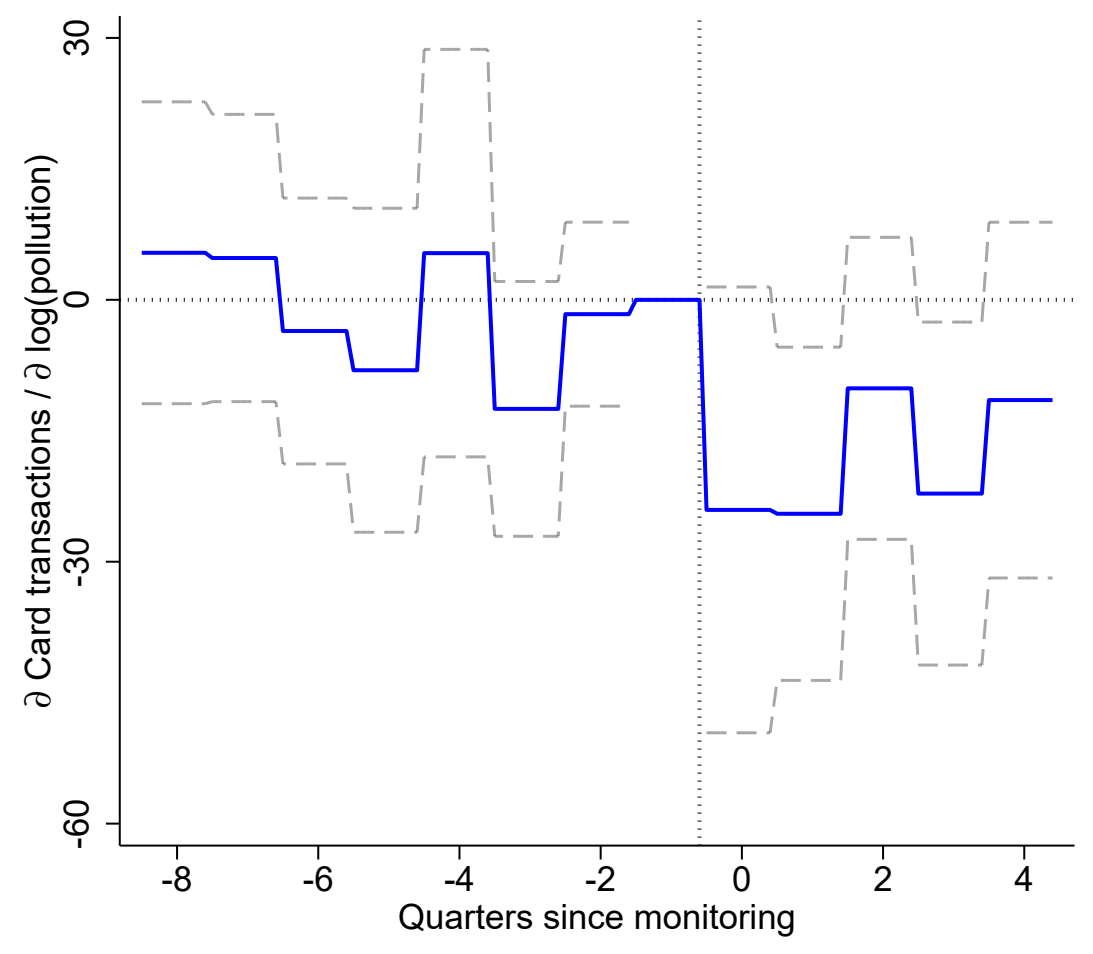

Notes: The figure shows coefficients from a regression of city-weekly band card transaction frequencies per 10,000 active cards on satellite-based pollution (in logarithm) as a function of quarters since the completion of the monitoring program. Since all transactions in our data occurred at physical stores, these transactions can be used as a proxy for outdoor trips. The shopping-pollution gradient, therefore, serves as a measure of avoidance behavior. Event quarter -1 is normalized to 0. Each line segment represents a coefficient estimate. The underlying regression controls for city, week-of-year, and year fixed effects, and city-specific time trends. Dashed lines show 95\% confidence interval constructed from standard errors clustered at the city level. 


\section{Figure 7: Changes in Defensive Spending - Air Purifier Sales in 50 Cities}

(a) National air purifier sales

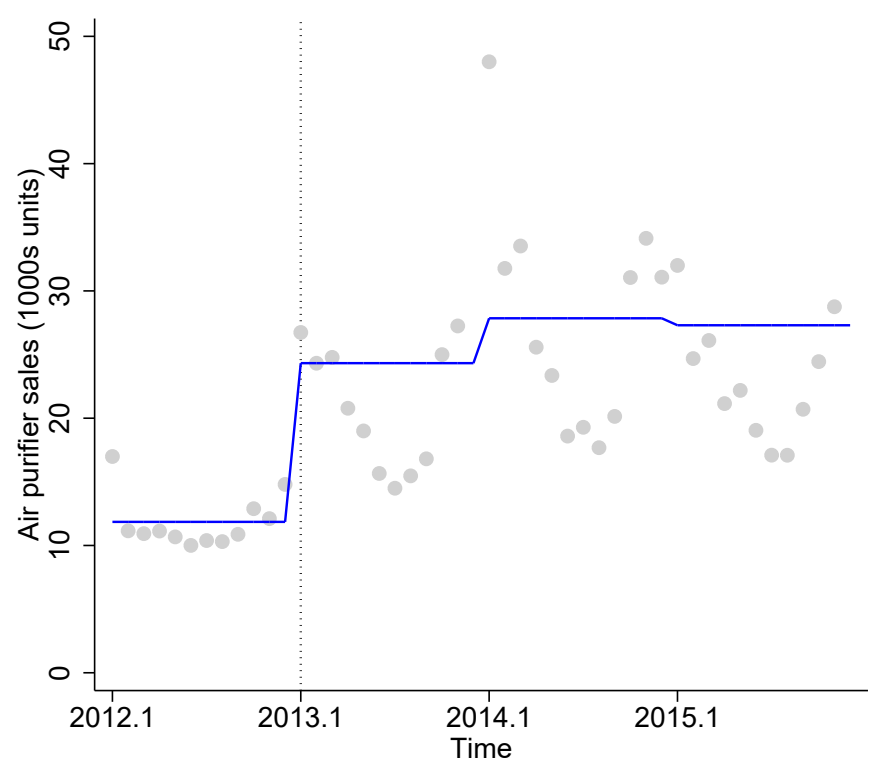

(b) City-level sales before and after monitoring

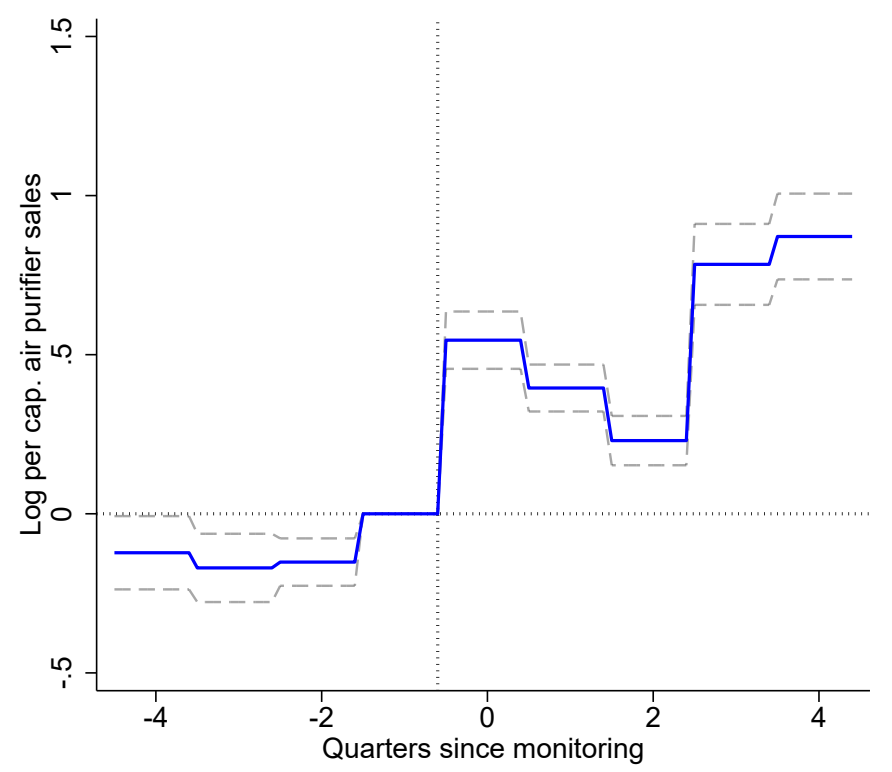

Notes: Panel (a) plots raw monthly trends in national air purifier sales from offline venues. The graph omits two dots with exceptionally high sales for readability. These dots correspond to December 2013 ( sales $=61,605$ units) and December 2015 (sales $=74,352$ units). Line shows annual averages. Panel (b) plots log per capita air purifier sales as a function of quarters since the completion of the monitoring program in a given city. Event quarter -1 is normalized to 0 . The underlying regression contains no control variables; see Appendix Figure E.6 for versions with fixed effects controls. We report four quarters of pre-treatment coefficients because the air purifier data are available since January 2012, one year prior to the initial rollout of the monitoring program. Dashed lines show $95 \%$ confidence interval constructed from standard errors clustered at the city level. 
Figure 8: Changes in Health Damage: Mortality-Pollution Gradient

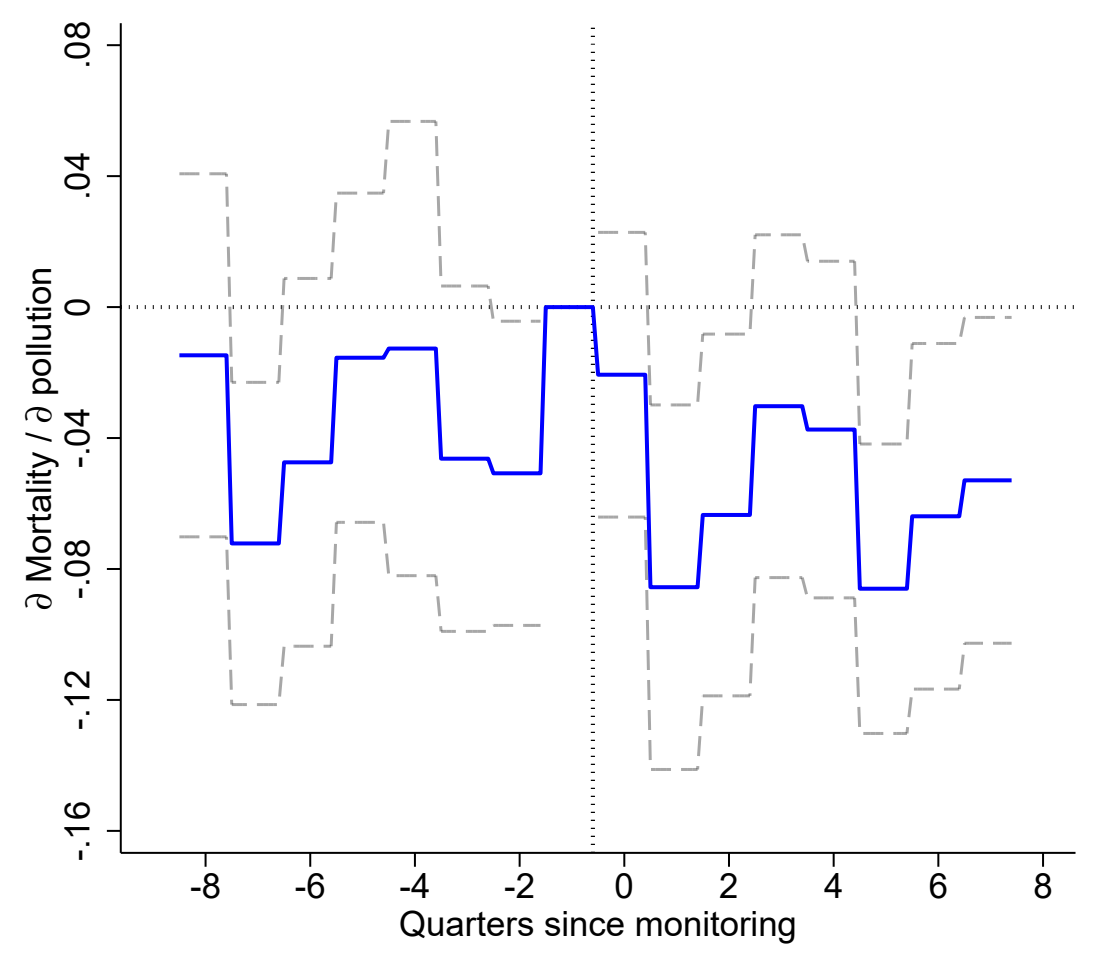

Notes: This figure shows coefficients from a regression of log mortality rate on log satellite-based pollution as a function of quarters since the completion of the monitoring program in a given city. Event quarter -1 is normalized to 0. Each line segment represents a coefficient estimate. The underlying regression controls for city, week-of-year, and year fixed effects, and city-specific time trends. Dashed lines show $95 \%$ confidence interval constructed from standard errors clustered at the city level. 
Table 1: Summary Statistics

\begin{tabular}{lccccc}
\hline & & & & & \\
& Cities available & Years available & Frequency & Mean & SD \\
\hline People's Daily "smog" articles (count per week) & 313 & $2011.1-2016.4$ & weekly & 0.031 & 0.310 \\
Baidu web "smog' searches (raw index) & 292 & $2011.1-2016.4$ & weekly & 285.3 & 916.5 \\
Expressed environmental concerns (scale 1-10) & 126 & $2012,2014,2016,2018$ & panel survey & 5.85 & 2.68 \\
Air purifier purchases (units per capita) & 50 & $2012.1-2016.4$ & monthly & 0.063 & 0.090 \\
Bank card transactions: all (swipes per 10,000 cards) & 334 & $2011.1-2016.4$ & weekly & 869.1 & 347.6 \\
Bank card transactions: deferrable (swipes per 10,000 cards) & 334 & $2011.1-2016.4$ & weekly & 314.4 & 161.3 \\
Mortality rate: general (deaths per 100,000) & 131 & $2011.1-2016.12$ & weekly & 10.36 & 3.46 \\
Mortality rate: cardiorespiratory (deaths per 100,000) & 131 & $2011.1-2016.12$ & weekly & 5.63 & 2.58 \\
Aerosol optical depth (pollution index) & 334 & $2006.1-2016.12$ & weekly & 0.550 & 0.419 \\
\hline
\end{tabular}

Notes: Except for air purifier purchases, all other data sets are national or nationally representative in scope. The 50 cities with air purifier purchases cover $28 \%$ of the total population. Self-reported environmental concerns are from the China Family Panel Studies, which is a nationally representative, bi-annual longitudinal survey. Bank card transactions cover all cities and account for $59 \%$ of national consumption. The 131 cities with mortality data constitute a $5 \%$ representative sample of the total population. Satellite pollution data covers the entire nation. 
Table 2: Changes in Avoidance Behavior: Shopping-Pollution Gradient

\begin{tabular}{lcccc}
\hline & $(1)$ & $(2)$ & $(3)$ & $(4)$ \\
\cline { 2 - 5 } & & & & \\
Panel A. All transactions & & & & \\
\hline \multirow{2}{*}{$\log ($ Pollution $)$} & 5.78 & 3.74 & 2.38 & 4.75 \\
& $(3.72)$ & $(4.08)$ & $(3.37)$ & $(3.55)$ \\
$\log ($ Pollution $) \times 1($ after monitoring) & -10.50 & -13.30 & -8.22 & -12.17 \\
& $(4.45)$ & $(5.19)$ & $(4.59)$ & $(5.24)$ \\
& {$[0.048]$} & {$[0.032]$} & {$[0.136]$} & {$[0.056]$}
\end{tabular}

Panel B. Merchant type: deferrable (supermarket, dining, entertainment)

$\begin{array}{lcccc}\log (\text { Pollution }) & 3.77 & 3.31 & 2.87 & 3.69 \\ & (1.50) & (1.77) & (1.38) & (1.65) \\ \log (\text { Pollution }) \times 1 \text { (after monitoring) }) & -8.33 & -9.02 & -6.51 & -8.69 \\ & (1.57) & (2.22) & (2.04) & (2.31) \\ & {[0.000]} & {[0.00]} & {[0.0140]} & {[0.010]}\end{array}$

Panel C. Merchant type: non-deferrable (all other categories)

\begin{tabular}{lcccc}
\hline Log(Pollution) & & & & \\
& 2.01 & 0.43 & -0.50 & 1.06 \\
& $(2.53)$ & $(2.65)$ & $(2.28)$ & $(2.32)$ \\
$\log ($ Pollution) $\times 1$ (after monitoring) & -2.17 & -4.29 & -1.71 & -3.48 \\
& $(3.24)$ & $(3.35)$ & $(2.98)$ & $(3.53)$ \\
& {$[0.536]$} & {$[0.228]$} & {$[0.598]$} & {$[0.400]$} \\
& & & & \\
FEs: city, city-linear time trends & $\checkmark$ & $\checkmark$ & $\checkmark$ & $\checkmark$ \\
FEs: week-of-year & $\checkmark$ & & & \\
FEs: year & $\checkmark$ & & & \\
FEs: week-of-sample & & $\checkmark$ & $\checkmark$ & \\
FEs: region $\times$ year & & & $\checkmark$ & $\checkmark$ \\
FEs: region $\times$ week-of-sample & & & & $\checkmark$ \\
\hline
\end{tabular}

Notes: Number of observations is 83,122 . " $\log ($ Pollution)" is logged AOD in a city $\times$ week. Dependent variable is city $\times$ weekly bank card transactions per 10,000 active cards. Mean of the dependent variable is 869.1 (panel A), 314.4 (panel B), and 554.8 (panel C). "Region" is a conventional partition of cities by location: north (36 cities), northeast (38 cities), east (105 cities), central south (81 cities), southwest (54 cities), and northwest (52 cities). Standard errors clustered at the city level reported in parentheses. Family-wise adjusted p-values based on the step-down resampling procedure of Westfall and Young (1993) are in brackets. 
Table 3: Changes in the Health Damage: Mortality-Pollution Gradient

$(1)$

$(2)$

$(3)$

Panel A. General mortality

\begin{tabular}{lcccc}
\hline $\log ($ Pollution $)$ & 0.0069 & 0.0059 & 0.0039 & 0.0020 \\
& $(0.0062)$ & $(0.0068)$ & $(0.0049)$ & $(0.0057)$ \\
$\log ($ Pollution $) \times 1$ (after monitoring) & -0.0228 & -0.0190 & -0.0159 & -0.0126 \\
& $(0.0069)$ & $(0.0076)$ & $(0.0064)$ & $(0.0068)$ \\
& {$[0.006]$} & {$[0.044]$} & {$[0.038]$} & {$[0.134]$}
\end{tabular}

Panel B. Causes of death: cadiorespiratory

$\begin{array}{lcccc}\log (\text { Pollution }) & 0.0113 & 0.0104 & 0.0097 & 0.0091 \\ & (0.0070) & (0.0075) & (0.0062) & (0.0072) \\ \log (\text { Pollution }) \times 1(\text { after monitoring }) & -0.0312 & -0.0257 & -0.0254 & -0.0238 \\ & (0.0079) & (0.0083) & (0.0074) & (0.0083) \\ & {[0.002]} & {[0.020]} & {[0.010]} & {[0.022]}\end{array}$

Panel C. Causes of death: non-cadiorespiratory

\begin{tabular}{lcccc}
\hline Log(Pollution) & -0.0003 & -0.0018 & -0.0043 & -0.0086 \\
& $(0.0067)$ & $(0.0073)$ & $(0.0061)$ & $(0.0071)$ \\
$\log ($ Pollution) $\times 1$ (after monitoring) & -0.0130 & -0.0096 & -0.0052 & -0.0012 \\
& $(0.0088)$ & $(0.0095)$ & $(0.0088)$ & $(0.0100)$ \\
& {$[0.246]$} & {$[0.372]$} & {$[0.560]$} & {$[0.902]$} \\
Panel D. Causes of death: injury & & & & \\
\hline & & & & \\
Log(Pollution) & -0.0132 & -0.0172 & -0.0191 & -0.0209 \\
& $(0.0080)$ & $(0.0085)$ & $(0.0082)$ & $(0.0097)$ \\
Log(Pollution) $\times 1$ (after monitoring) & 0.0002 & 0.0125 & 0.0169 & 0.0204 \\
& $(0.0089)$ & $(0.0100)$ & $(0.0101)$ & $(0.0115)$ \\
& {$[0.990]$} & {$[0.372]$} & {$[0.178]$} & {$[0.142]$} \\
FEs: city, city-linear time trends & $\checkmark$ & $\checkmark$ & $\checkmark$ & $\checkmark$ \\
FEs: week-of-year & $\checkmark$ & & & \\
FEs: year & $\checkmark$ & & & \\
FEs: week-of-sample & & $\checkmark$ & $\checkmark$ & $\checkmark$ \\
FEs: region $\times$ year & & & $\checkmark$ & \\
FEs: region $\times$ week-of-sample & & & & \\
\hline
\end{tabular}

Notes: Numbers of observations are: 36,369 (panel A), 36,226 (panel B), 36,262 (panel C), and 32,621 (panel D). "Log(Pollution)" is logged AOD in a city $\times$ week. Dependent variable is city $\times$ weekly logged mortality rate. "Region" is a conventional partition of cities by location: north (36 cities), northeast (38 cities), east (105 cities), central south (81 cities), southwest (54 cities), and northwest (52 cities). Standard errors clustered at the city level are reported in parentheses.

Family-wise adjusted p-values based on the step-down resampling procedure of Westfall and Young (1993) are in brackets. 
Table 4: Changes in the Health Damage: Age-Specific Mortality-Pollution Gradient

(1)

$(2)$

(3)

(4)

Panel A. Age groups: infants

\begin{tabular}{lcccc}
\hline $\log ($ Pollution $)$ & 0.0104 & 0.0097 & 0.0032 & 0.0033 \\
& $(0.0074)$ & $(0.0074)$ & $(0.0075)$ & $(0.0097)$ \\
$\log ($ Pollution $) \times 1$ (after monitoring) & -0.0044 & -0.0052 & 0.0070 & 0.0009 \\
& $(0.0116)$ & $(0.0130)$ & $(0.0137)$ & $(0.0161)$ \\
& {$[0.766]$} & {$[0.918]$} & {$[0.692]$} & {$[0.950]$}
\end{tabular}

Panel B. Age groups: 1-14

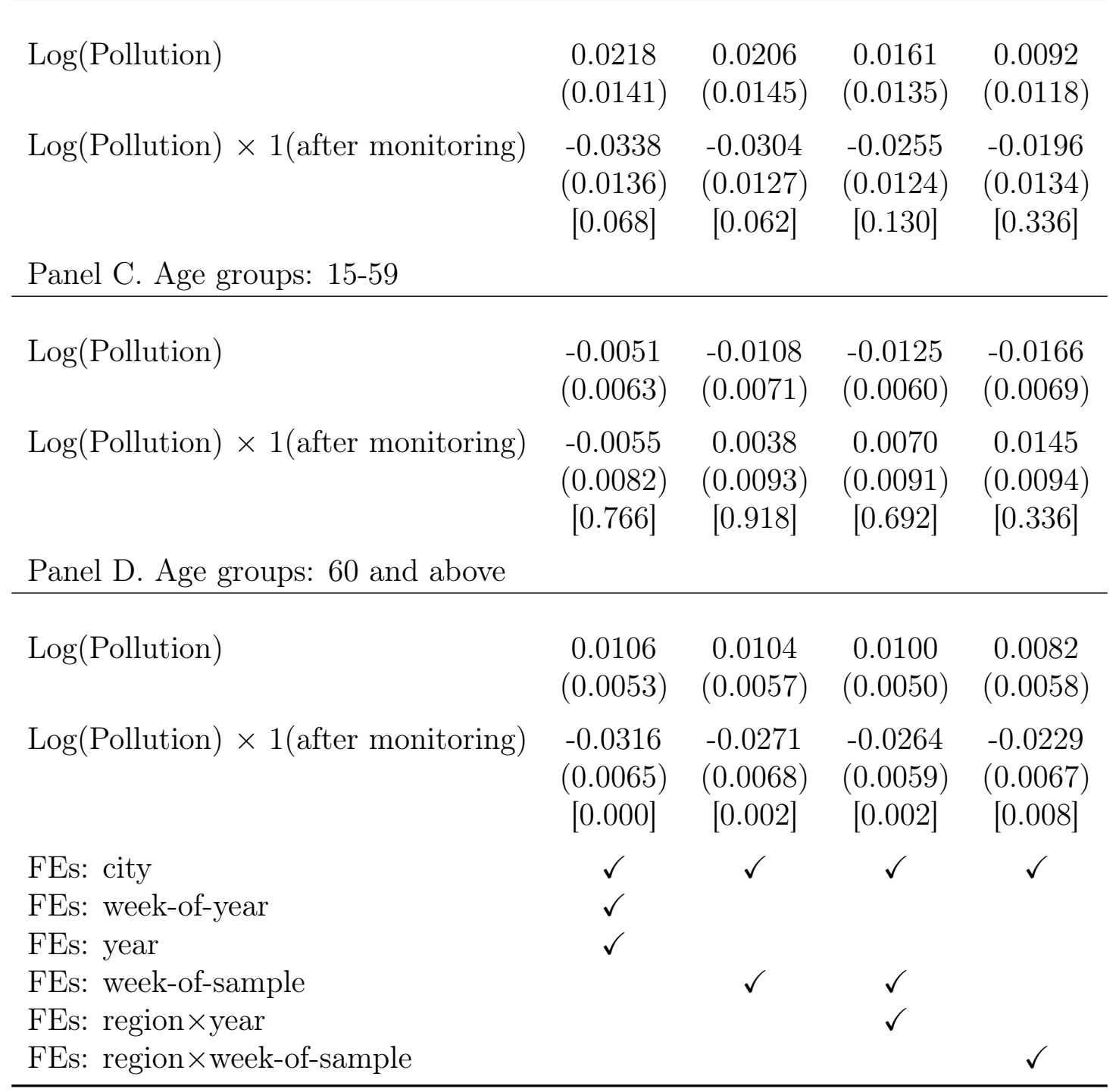

Notes: Numbers of observations are: 14,060 (panel A), 12,626 (panel B), 35,932 (panel C), and 36,272 (panel D). "Log(Pollution)" is logged AOD in a city $\times$ week. Dependent variable is city $\times$ weekly logged mortality rate. "Region" is a conventional partition of cities by location: north (36 cities), northeast (38 cities), east (105 cities), central south (81 cities), southwest (54 cities), and northwest (52 cities). Standard errors clustered at the city level are reported in parentheses.

Family-wise adjusted p-values based on the step-down resampling procedure of Westfall and Young (1993) are in brackets. 


\section{Online Appendix for \\ From Fog to Smog: the Value of Pollution Information}

Panle Jia Barwick, Shanjun Li, Liguo Lin, Eric Zou

This appendix provides supplementary material to the paper "From Fog to Smog: the Value of Pollution Information." Section A describes the driving factors that influenced China's dramatic shift in environmental regulations surrounding 2012. Section B presents a theoretical model on the role of the monitoring program that reduces the wedge between individuals' perceived and experienced air pollution. Section $\mathrm{C}$ proves that the key parameter can be consistently estimated via OLS under assumptions 1 and 2 as stated in Section 4.2. Section D provides more details on several empirical analyses, including program rollout, a data-driven method to identify outdoor trips, private sources for pollution information, IV and synthetic analyses of mortality outcomes, and robustness results using annual data (instead of weekly data).

\section{Appendix A: Policy Shift Timeline and Driving Factors}

Appendix Table E.1 provides a chronology of important events related to the policy shift on $\mathrm{PM}_{2.5}$ regulation discussed in Section 2.1 and the implementation of the monitoring and disclosure program. The shift of the government's stance on regulating $\mathrm{PM}_{2.5}$ is reflected by the change from the first to the second draft of the National Ambient Air Quality Standards amendment published for public comments on November 2010 and November 2011, respectively. While the first draft deemed China not yet ready to implement national standards for $\mathrm{PM}_{2.5}$, the second draft a year later added the national standards for $\mathrm{PM}_{2.5}$.

As household income rose rapidly and a large middle class emerged in China during the past two decades, the demand for better environmental quality and quality of life in general increased (Dasgupta et al., 2002; Kahn and Zheng, 2016). Together with the important changes in economic and social conditions, the following three factors likely played a key role in propelling the dramatic shift in the stance of the Chinese government on $\mathrm{PM}_{2.5}$ monitoring and disclosure, and on air pollution regulations in general.

First, the environmental science community has been working behind the scene to push for more stringent environmental regulations and transparency in China. As a large body of evidence emerged regarding the harmful health impacts of $\mathrm{PM}_{2.5}$ in the past several decades (Pope and Dockery, 2012; Landrigan et al., 2018) and recognized by policymakers around the world, many countries have amended their ambient air quality standards to 
include $\mathrm{PM}_{2.5}$ as a primary pollutant (e.g., U.S. in 1997, EU in 1999, and Japan in 2009). Environmental scientists in China have been pushing for the regulation for $\mathrm{PM}_{2.5}$ through academic conferences and policy dialogues, according to our conversation with Jiming Hao, Professor and a former Dean of the School of Environment at Tsinghua University and one of the pioneers in China's environmental regulations and pollution prevention. ${ }^{44}$ In the early 2000s, environmental scientists organized international conferences on air pollution and $\mathrm{PM}_{2.5}$ in China in order to draw attention from and educate policymakers. Nevertheless, policymakers were predominantly concerned about economic growth and did not see the benefit of educating the general public when the government was not yet ready to bring the pollution problem under control. This concern perhaps underlies the views stated in the first draft of the NAAQS amendment released in November 2010. While the draft recognized that $\mathrm{PM}_{2.5}$ had become a major pollutant in many areas in China, it considered standards set by the WHO and developed countries too aggressive for China and deemed that China was not ready to set national $\mathrm{PM}_{2.5}$ standards.

Second, the U.S. Embassy in Beijing is likely another important catalyst behind the shift. In early 2008, the embassy installed a rooftop air quality monitor and reported hourly $\mathrm{PM}_{2.5}$ readings through the Twitter account @beijingair in order to advise U.S. citizens who travel in China before the upcoming summer Olympics. The $\mathrm{PM}_{2.5}$ readings were often in conflict with the Chinese government's official report on air quality, especially during the extreme pollution episodes in winter. For example, on November 19, 2010, the Twitter account @beijingair reported $\mathrm{PM}_{2.5}$ over 500 and described the air pollution as "crazily bad", while China's official assessment of air quality based on API was "mildly polluted" $\left(\mathrm{PM}_{2.5}\right.$ was not incorporated in API). On the one hand, the discrepancy caused political tensions: China's Ministry of Foreign Affairs asked the U.S. Embassy to stop reporting $\mathrm{PM}_{2.5}$ because it "caused confusion and undesirable social consequences." On the other hand, this prompted environmental activists in China to call for more transparency on pollution information. The real-time data from the U.S. Embassy's air quality monitor in Beijing forced the Chinese government to confront the issue more seriously than otherwise. The MEP had to make a choice: either continue to dispute the U.S. Embassy's data and contest its data release, or provide their own data that the general public would trust.

Third, environmental NGOs and social influencers have played an important role in raising the awareness of the general public and exerting pressure on policymakers. Their role is facilitated and amplified by the emergence of social media (notably through Weibo in China,

\footnotetext{
${ }^{44}$ In 2015, Professor Hao was awarded the Haggen-Smit Clean Air Award by the California Air Resources Board for his contribution to air pollution control and understanding PM pollution in major cities in China. When he visited Cornell University in May 2019, our research team met with him to understand the context of the policy shift in our study and more broadly the history of environmental regulations in China.
} 
as Facebook and Twitter were banned in China) and the wide adoption of information technologies. Motivated by severe pollution episodes and the lack of transparency and reporting of air pollution levels, the campaign "Measuring Air Quality for our Motherland" in the Fall of 2011 was a concerted effort mobilized through social media Weibo by environmental NGOs and influential activists. The campaign called for setting national $\mathrm{PM}_{2.5}$ standards and implementing accurate monitoring and reporting of air quality by the government. Sensitive to the cultural and political environment, environmental NGOs in China often avoid the role of government watchdogs and position them as educators and partners in order to bring about changes more effectively. Similarly, the campaign made a plea for the government to continue watching out for people's welfare due to the detrimental health impacts of air pollution especially $\mathrm{PM}_{2.5}$. This campaign was considered a milestone and a great success in the discourse of civic participation in China's environmental governance (Fedorenko and Sun, 2016), contributing to the release of the second draft of the NAAQS amendment in November 2011 where $\mathrm{PM}_{2.5}$ was added as a primary pollutant to be regulated.

\section{Appendix B: Theoretical Model}

Classical economic theory argues that the value of information stems from the fact that information as an input to the decision process can help economic agents make better decisions - for example, by resolving market uncertainty in demand and supply conditions (Stigler, 1961, 1962) or technological uncertainty in investment and production decisions (Lave, 1963; Hirshleifer, 1971). Access to pollution information affects the behavior of informed individuals who could take measures to reduce the harm from pollution. In this section, we present a stylized model to illustrate how the monitoring program affects individual behavior and utility by incorporating the elements of information economics (Hirshleifer, 1971; Hilton, 1981) into a classical model of health demand and production (Grossman, 1972; Harrington and Portney, 1987)..

\section{B.1 Model Setup}

Individuals derive utility $U(x, h)$ from the consumption of a numeraire good $x$, whose price is normalized to one, and health stock $h$. Health stock depends on both the pollution level $c$ and the extent of avoidance $a$ (individuals' actions that mitigate the negative impact of pollution): $h=h(c, a)$.

Individuals face a budget constraint that is given by: $I+w \cdot g(h) \geq x+p_{a} \cdot a$, where $I$ is non-labor income, and $w$ is the wage rate. Hours worked is denoted by $g(h)$ and is a 
function of the health stock. ${ }^{45}$ Individuals allocate their wage and non-wage income between consumption and engaging in avoidance behavior $a$, where $p_{a}$ is the associated price (e.g., the cost of an air purifier or medication). We use $a$ to include broadly defined (costly) adaptation behavior. ${ }^{46}$ Dynamics and savings are assumed away to ease exposition.

Under imperfect information on pollution, consumers may or may not know the real pollution level $c$. They maximize utility by choosing the optimal consumption $x$ and defensive investment $a$ based on the perceived pollution level $c_{0}$ :

$$
\begin{aligned}
\max _{x, a} U(x, h) & \\
\text { s.t. } I+w \cdot g(h) & \geq x+p_{a} \cdot a \\
h & =h\left(c_{0}, a\right)
\end{aligned}
$$

The health function $h=h\left(c_{0}, a\right)$ in the optimization can be viewed as an ex ante health function upon which consumers rely for decisions before the health outcome is realized. It is different from the ex post health outcome $h=h(c, a)$ experienced by consumers. This difference gives rise to the discrepancy between the (ex ante) decision utility and the (ex post) experience utility as described in Bernheim and Rangel (2009) and Allcott (2013).

Let avoidance under the perceived pollution $c_{0}$ be denoted by $a\left(c_{0}\right)$. Individuals' wage income is determined by the actual pollution level $c$ and avoidance $a\left(c_{0}\right): w \cdot g\left[h\left(c, a\left(c_{0}\right)\right)\right]$. Let $x\left(c, c_{0}\right)$ denote consumption of the numeraire good. The experience utility based on the perceived pollution prior to the monitoring program is:

$$
U\left[x\left(c, c_{0}\right), h\left(c, a\left(c_{0}\right)\right)\right] \equiv V\left(c, c_{0}\right)
$$

where $V(\cdot, \cdot)$ denotes the indirect utility: the first argument is the actual pollution $c$, and the second argument is the perceived pollution level $c_{0}$. To examine the behavioral changes associated with and the welfare impacts of the monitoring program, we make the following assumptions:

Assumption A1 Health stock is bounded and decreases in pollution and increases in avoidance: $\frac{\partial h}{\partial c} \leq 0$, and $\frac{\partial h}{\partial a} \geq 0$. In addition, the marginal health benefit of avoidance is

\footnotetext{
${ }^{45}$ The effect of health on wage income captures the impact of pollution on labor supply or productivity as documented in Graff Zivin and Neidell (2012); Hanna and Oliva (2015); Chang et al. (2019); He, Liu and Salvo (2019).

${ }^{46}$ Examples include reducing outdoor activities (Zivin and Neidell, 2009; Saberian, Heyes and Rivers, 2017), engaging in defensive spending (e.g., buying face masks and air purifiers) (Ito and Zhang, 2018; Zhang and $\mathrm{Mu}, 2018$ ), and making choices to change residential locations or migrate (Chay and Greenstone, 2005; Banzhaf and Walsh, 2008; Bayer, Keohane and Timmins, 2009; Chen, Oliva and Zhang, 2017).
} 
decreasing: $\frac{\partial^{2} h}{\partial a^{2}} \leq 0$. This assumption ensures that people do not engage in an unreasonable amount of avoidance behavior. Similarly, we assume that hours worked increases in health, but at a decreasing rate: $\frac{d g}{d h} \geq 0, \frac{d^{2} g}{d h^{2}} \leq 0$. Finally, the worse the pollution, the larger the marginal health benefit of avoidance: $\frac{\partial^{2} h}{\partial a \partial c} \geq 0$. The health benefit of avoidance is likely much higher when pollution is severe than when it is modest.

We focus on interior solutions for the optimal level of avoidance behavior $a .{ }^{47}$ The assumption of $\frac{\partial^{2} h}{\partial a \partial c} \geq 0$ is crucial in delivering "complementarity" between pollution and avoidance: the higher the pollution, the more intense avoidance is likely to be. At low levels of pollution, the marginal health benefit of avoidance $\frac{\partial h}{\partial a}$ is likely to be limited. As pollution elevates, higher marginal benefits induce individuals to engage in more avoidance to mitigate the health and wage impact of pollution. There are many low-cost defensive mechanisms. For example, avoiding outdoor activities at times of high $\mathrm{PM}_{2.5}$, wearing facial masks, or purchasing air purifiers are all cheap and effective defensive mechanisms.

Assumption A2 Utility is quasi-linear $U(x, h)=x+u(h)$ and increases in health at a decreasing rate: $\frac{\partial U}{\partial h} \geq 0, \frac{\partial^{2} U}{\partial h^{2}} \leq 0$. Quasi-linear utility functions are commonly used in the literature and help to simplify the exposition.

Assumption A3 Let $c_{0}$ denote individuals' perception of air pollution before the monitoring program. We assume that $c_{0}<c$, that is, the perceived level of pollution is lower than the actual level. ${ }^{48}$ Another interpretation of Assumption 3 is that people underestimate the negative health impact of pollution. Pollution concentration $c$ is assumed to be perfectly observed following the implementation of the program.

Proposition 1. Under assumptions A1-A3, the monitoring program is predicted to result in the following impacts:

1. Avoidance behavior increases: $a(c)>a\left(c_{0}\right)$

2. Health improves and the (downward slopping) health-pollution response curve flattens:

$$
h(c, a(c))>h\left(c, a\left(c_{0}\right)\right),\left.\frac{d h}{d c}\right|_{c=c_{0}} \geq\left.\frac{d h}{d c}\right|_{c>c_{0}}
$$

3. Indirect utility increases: $V(c, c)>V\left(c, c_{0}\right)$

\footnotetext{
${ }^{47} \mathrm{~A}$ necessary condition for an interior solution is $\left.w \cdot \frac{d g}{d h} \cdot \frac{\partial h}{\partial a}\right|_{a=0}>p_{a}$.

${ }^{48} \mathrm{An}$ alternative assumption to $c_{0}<c$, is that the monitoring program reduces the price of avoidance $p_{a}$. This also delivers Proposition 1.
} 
Appendix B.3 provides the proof. The theoretical model predicts that following the implementation of the monitoring program, individuals engage in more pollution avoidance, which, in turn, reduces the health damages from pollution and increases consumer welfare. Our empirical analysis provides empirical tests on the first two predictions, and uses the third prediction to quantify the value of the monitoring program.

\section{B.2 Value of Information}

To derive the value of information (VOI), recall that:

$$
V(c, c)=U[x, h(c, a(c))]+\lambda\left\{I+w \cdot g[h(c, a(c))]-x-p_{a} \cdot a(c)\right\}
$$

where $V(c, c)$ denotes the indirect utility when individuals correctly perceive pollution, and avoidance is chosen optimally according to the following condition:

$$
\left[U_{h}(c, a)+\lambda \cdot w \cdot g_{h}(h(c, a))\right] \frac{\partial h(c, a)}{\partial a}-\lambda p_{a}=0
$$

The indirect utility before the monitoring program is:

$$
V\left(c, c_{0}\right)=U\left[x, h\left(c, a\left(c_{0}\right)\right)\right]+\lambda\left\{I+w \cdot g\left[h\left(c, a\left(c_{0}\right)\right)\right]-x-p_{a} \cdot a\left(c_{0}\right)\right\}
$$

The key difference between $V(c, c)$ and $V\left(c, c_{0}\right)$ is in the choice of avoidance: $a(c)$ is determined by Equation (B.1) rather than Equation (B.3). To derive the value of information, we apply the Taylor's series expansion to the indirect utility function $V(c, c)$ at the second argument $c=c_{0}: V(c, c)=V\left(c, c_{0}\right)+\frac{\partial V}{\partial c_{0}}\left(c-c_{0}\right)+o_{p}\left(c-c_{0}\right)$, where $o_{p}\left(c-c_{0}\right)$ denotes higher order terms of $\left(c-c_{0}\right)$. The value of information is therefore:

$$
\begin{aligned}
V O I & =V(c, c)-V\left(c, c_{0}\right) \\
& =\left\{U_{h} \cdot \frac{\partial h}{\partial a} \cdot \frac{\partial a}{\partial c_{0}}+\lambda \cdot w \cdot g_{h} \cdot \frac{\partial h}{\partial a} \cdot \frac{\partial a}{\partial c_{0}}-\lambda \cdot p_{a} \cdot \frac{\partial a}{\partial c_{0}}\right\}\left(c-c_{0}\right)+o_{p}\left(c-c_{0}\right)
\end{aligned}
$$

There are three terms in the curly bracket. The first refers to changes in utility as health improves from the avoidance behavior. The second denotes changes in wage income due to pollution impact on effective work hours or productivity. The third term in the curly bracket captures the cost of taking additional avoidance measures such as buying air purifiers or changing outdoor activities. Our empirical analysis quantifies the magnitude of the terms in the curly bracket. 


\section{B.3 Proof of Proposition 1}

Individuals choose optimal consumption $x$ and defensive investment $a$ to maximize utility under the perceived pollution level $c_{0}$ as described in Section B.1. The Lagrangian equation is:

$$
L=U\left(x, h\left(c_{0}, a\right)\right)+\lambda\left[I+w \cdot g\left(h\left(c_{0}, a\right)\right)-x-p_{a} \cdot a\right]
$$

where $\lambda$ is the Lagrange multiplier and denotes the marginal utility per dollar. The first order conditions are:

$$
\begin{aligned}
& \frac{\partial L}{\partial x}=0 \Rightarrow U_{x}-\lambda=0 \\
& \frac{\partial L}{\partial a}=0 \Rightarrow\left(U_{h}+\lambda \cdot w \cdot g_{h}\right) \frac{\partial h\left(c_{0}, a\right)}{\partial a}-\lambda p_{a}=0 \\
& \frac{\partial L}{\partial \lambda}=0 \Rightarrow I+w \cdot g(h)-x-p_{a} \cdot a=0
\end{aligned}
$$

where $U_{x}, U_{h}$, and $g_{h}$ denote partial derivatives. We first show that under Assumptions 1-3, optimal avoidance (weakly) increases in perceived pollution:

$$
\frac{d a}{d c} \geq 0
$$

Let $f$ denote the first order condition w.r.t avoidance (equation B.3):

$$
f=\left(U_{h}+\lambda \cdot w \cdot g_{h}\right) \frac{\partial h}{\partial a}-\lambda p_{a}=0
$$

Applying the implicit function theorem to $f$, we obtain:

$$
\frac{d a}{d c}=-\frac{\partial f / \partial c}{\partial f / \partial a}=-\frac{\left[U_{h h}+\lambda \cdot w \cdot g_{h h}\right] \cdot \frac{\partial h}{\partial c} \cdot \frac{\partial h}{\partial a}+\left(U_{h}+\lambda \cdot w \cdot g_{h}\right) \cdot \frac{\partial^{2} h}{\partial a \partial c}}{\left(U_{h h}+\lambda \cdot w \cdot g_{h h}\right) \cdot\left(\frac{\partial h}{\partial a}\right)^{2}+\left(U_{h}+\lambda \cdot w \cdot g_{h}\right) \cdot \frac{\partial^{2} h}{\partial a^{2}}}=-\frac{A+B}{C+D}
$$

where $U_{h x}, U_{h h}, g_{h h}$ are second order derivatives. Under the assumption of diminishing marginal utility, decreasing marginal labor product of health, and decreasing health benefit of avoidance, $C+D \leq 0 .{ }^{49}$ Similarly, $A+B \geq 0$. Hence, avoidance increases weakly in (perceived) pollution. The key assumption for this result is $\partial h^{2} / \partial a \partial c \geq 0$. When pollution deteriorates, avoidance restores health more effectively (that is, the marginal benefit of avoidance is large with bad pollution). After the monitoring program, individuals observe the actual pollution $c$, which is higher than previously perceived level, $c_{0}$. The above analysis

\footnotetext{
${ }^{49}$ At the optimal $a$ and $X, U_{h}+\lambda \cdot w \cdot g(h)>0$ by construction. In addition, $U_{h h}, g_{h h}, \partial^{2} h / \partial a^{2}<0$. Another way to show $C+D \leq 0$ is that this is the second order condition for the optimal avoidance.
} 
indicates that individuals would increase the level of avoidance post the policy intervention:

$$
a(c) \geq a\left(c_{0}\right)
$$

As the marginal health benefit of avoidance is positive from Assumption (A1) in Section B.1, the health condition improves with avoidance:

$$
h(c, a(c)) \geq h\left(c, a\left(c_{0}\right)\right) .
$$

Due to the lack of real-time information on pollution prior to the monitoring program, perceived pollution $c_{0}$ is unlikely to fully respond to day-to-day changes in actual pollution. The total derivative of health w.r.t. pollution is:

$$
\left.\frac{d h}{d c}\right|_{c_{0}}=\frac{\partial h}{\partial c}+\frac{\partial h}{\partial a} \cdot \frac{d a}{d c_{0}} \cdot \frac{d c_{0}}{d c}
$$

where $0<d c_{0} / d c<1$. Post the monitoring program, the perceived pollution is equal to the actual pollution and individuals can engage in effective avoidance to moderate the negative impact of pollution. The total derivative of health w.r.t. pollution is:

$$
\left.\frac{d h}{d c}\right|_{c}=\frac{\partial h}{\partial c}+\frac{\partial h}{\partial a} \cdot \frac{d a}{d c} \geq\left.\frac{d h}{d c}\right|_{c_{0}}
$$

Lastly, let $V(c, c)$ denote the indirect utility when individuals accurately perceive pollution $c_{0}=c$. In that case, the experience utility and decision utility coincides. $V\left(c, c_{0}\right)$ is the utility achieved by maximizing the decision utility under perceived pollution of $c_{0}$. Since utility is maximized under full (and accurate) information, we have:

$$
V(c, c) \geq V\left(c, c_{0}\right) .
$$

\section{Appendix C: Identification of the Change in the Outcome- Pollution Gradient}

In this section, we restate the two key identification assumptions that are outlined in the paper and prove Proposition 2 (that the OLS estimate of $\beta$ in Equation (1) is consistent). Rewriting Equation (1):

$$
y_{i t}=\alpha p_{i t}+\beta p_{i t} d_{i t}+\boldsymbol{x}_{i t} \boldsymbol{\gamma}+\varepsilon_{i t},
$$


where $p_{i t}$ measures ambient air quality and could be correlated with $\varepsilon_{i t}$ due to unobservables or measurement error as discussed in the main text. $d_{i t}$ represents the treatment dummy and is equal to one after treatment based on the staggered rollout schedule. $\boldsymbol{x}_{c t}$ includes city attributes and other controls such as city and time fixed effects, and city-specific time trends. The key parameter of interest is $\beta$, the change in the slope of pollution-outcome relationship. To facilitate proof, we write Equation C.4 above in matrix form:

$$
\boldsymbol{y}=\alpha \boldsymbol{p}+\beta \boldsymbol{p} \circ \boldsymbol{d}+\boldsymbol{X} \boldsymbol{\gamma}+\boldsymbol{\varepsilon}
$$

where ' $\circ$ ' is an element-by-element product. $\boldsymbol{y}, \boldsymbol{p}, \boldsymbol{d}$, and $\varepsilon$ are $N$ by 1 vectors, $\boldsymbol{X}$ is a $N$ by $k$ matrix.

Assumption B1: $\varepsilon_{i t} \perp d_{i t} \mid \boldsymbol{x}_{i t}$. This assumption implies that conditioning on city attributes and other controls $\boldsymbol{x}_{c t}$, the treatment $d_{c t}$ is exogenous.

Assumption B2: $\quad d_{i t} \perp p_{i t} \mid \boldsymbol{x}_{i t}$. This assumption implies that the treatment status is independent of pollution levels conditioning on $\boldsymbol{x}_{c t}$ (i.e., variations in pollution unexplained by $\left.\boldsymbol{x}_{c t}\right) .{ }^{50}$

Assumption B1 is the standard conditional exogeneity assumption: the program rollout is as good as random conditional on the control variables. This assumption can be assessed via both institutional and econometric evidence (Section 4.3). We show that the assignment of cities to rollout wave largely follows city hierarchies and designated status that are determined long before the monitoring place came into place. We discuss other major environmental policies and argue that they have limited scope to confound our analysis. We present event study estimates, which allows us to assess whether there are any preexisting trends in the outcomes that could be indicative of endogeneity issues.

Assumption B2 ensures the nature of endogeneity is the same before versus after the monitoring program. One way to conceptualize it is to imagine a binary context in which Pollution indicates "high" vs. "low" pollution areas. Note that Equation (1) reduces to a difference-in-differences style setting that compares outcome in regions with high vs. low pollution, before vs. after policy introduction. The outcome-pollution gradient in this case is simply the difference in the outcomes experienced in areas with high and low pollution. Assumption B2 ensures that there are no compositional changes in regions that experience

\footnotetext{
${ }^{50}$ While we present Assumption B2 for ease of interpretation, the assumption is stronger than what we need to prove the consistency of $\beta$. As we show in the proof that follows, a sufficient condition is $\mathbb{E}\left[d_{i t} \mid \boldsymbol{M}_{\mathrm{x}} \boldsymbol{p}\right]=c$, where $\boldsymbol{M}_{\mathrm{x}}$ is the projection matrix; $\boldsymbol{M}_{\mathrm{x}} \boldsymbol{p}$ is the residual from OLS regression of $\boldsymbol{p}$ on $\boldsymbol{X}$; and $c$ is a constant.
} 
high or low levels of pollution after the policy introduction. In other words, the nature of the endogeneity in pollution does not change before and after the policy. Assumption B2 can be tested empirically as well (Section 4.3). In particular, we present balancing test in Appendix Table E.5 which shows that, conditional on fixed effects controls, the monitoring policy does not lead to significant changes in pollution levels.

We now demonstrate theoretically that the $\beta$ coefficient in Equation 1 is consistent under these two identification assumptions.

Proposition 2. Under Assumptions B1 and B2, the OLS estimate of $\beta$ in Equation (C.4) is consistent.

Proof of Proposition 2 Let $\boldsymbol{M}_{\mathrm{x}}$ denote the projection matrix: $\boldsymbol{M}_{\mathrm{x}}=\boldsymbol{I}-\boldsymbol{X}\left(\boldsymbol{X}^{\prime} \boldsymbol{X}\right)^{-1} \boldsymbol{X}^{\prime}$. Multiplying both sides of equation C.5 with $\boldsymbol{M}_{\mathrm{x}}$, we have:

$$
\boldsymbol{M}_{\mathrm{x}} \boldsymbol{Y}=\alpha \boldsymbol{M}_{\mathrm{x}} \boldsymbol{P}+\beta \boldsymbol{M}_{\mathrm{x}} \boldsymbol{p} \circ \boldsymbol{d}+\boldsymbol{M}_{\mathrm{x}} \boldsymbol{\varepsilon}
$$

where $\boldsymbol{M}_{\mathrm{x}} \boldsymbol{P}$ is an $N$ by 1 vector, the projection residual of $\boldsymbol{p}$ on $\boldsymbol{X}$. Collect the two key regressors in $\boldsymbol{Z}=\left[\boldsymbol{M}_{\mathrm{x}} \boldsymbol{p}, \boldsymbol{M}_{\mathrm{x}} \boldsymbol{p} \circ \boldsymbol{d}\right]$. Let the OLS estimates of $\alpha$ and $\beta$ be denoted as $\hat{\alpha}$ and $\hat{\beta}$.

$$
\begin{aligned}
\left(\begin{array}{c}
\hat{\alpha}-\alpha \\
\hat{\beta}-\beta
\end{array}\right)= & \left(\begin{array}{cc}
\left(\boldsymbol{M}_{\mathrm{x}} \boldsymbol{p} \circ \boldsymbol{d}\right)^{\prime}\left(\boldsymbol{M}_{\mathrm{x}} \boldsymbol{p} \circ \boldsymbol{d}\right) & -\left(\boldsymbol{M}_{\mathrm{x}} \boldsymbol{p}\right)^{\prime}\left(\boldsymbol{M}_{\mathrm{x}} \boldsymbol{p} \circ \boldsymbol{d}\right) \\
-\left(\boldsymbol{M}_{\mathrm{x}} \boldsymbol{p} \circ \boldsymbol{d}\right)^{\prime}\left(\boldsymbol{M}_{\mathrm{x}} \boldsymbol{p}\right) & \left(\boldsymbol{M}_{\mathrm{x}} \boldsymbol{p}\right)^{\prime}\left(\boldsymbol{M}_{\mathrm{x}} \boldsymbol{p}\right)
\end{array}\right)\left(\begin{array}{c}
\left(\boldsymbol{M}_{\mathrm{x}} \boldsymbol{p}\right)^{\prime}\left(\boldsymbol{M}_{\mathrm{x}} \boldsymbol{\epsilon}\right) \\
\left(\boldsymbol{M}_{\mathrm{x}} \boldsymbol{p} \circ \boldsymbol{d}\right)^{\prime}\left(\boldsymbol{M}_{\mathrm{x}} \boldsymbol{\epsilon}\right)
\end{array}\right) \\
& * \frac{1}{\operatorname{det}\left[\left(\boldsymbol{Z}^{\prime} \boldsymbol{Z}\right)\right]} .
\end{aligned}
$$

The probability limit of $(\hat{\beta}-\beta)$ converges to the following term multiplied by a constant:

$$
-\mathbb{E}\left[\left(\boldsymbol{M}_{\mathrm{x}} \boldsymbol{p} \circ \boldsymbol{d}\right)^{\prime}\left(\boldsymbol{M}_{\mathrm{x}} \boldsymbol{p}\right)\right] \mathbb{E}\left[\left(\boldsymbol{M}_{\mathrm{x}} \boldsymbol{p}\right)^{\prime}\left(\boldsymbol{M}_{\mathrm{x}} \boldsymbol{\epsilon}\right)\right]+\mathbb{E}\left[\left(\boldsymbol{M}_{\mathrm{x}} \boldsymbol{p}\right)^{\prime}\left(\boldsymbol{M}_{\mathrm{x}} \boldsymbol{p}\right)\right] \mathbb{E}\left[\left(\boldsymbol{M}_{\mathrm{x}} \boldsymbol{p} \circ \boldsymbol{d}\right)^{\prime}\left(\boldsymbol{M}_{\mathrm{x}} \boldsymbol{\epsilon}\right)\right]
$$

Assumption B2 implies that $E\left(\boldsymbol{d} \mid \boldsymbol{M}_{\mathrm{x}} \boldsymbol{p}\right)=c$. Hence:

$$
\mathbb{E}\left[\left(\boldsymbol{M}_{\mathrm{x}} \boldsymbol{p} \circ \boldsymbol{d}\right)^{\prime}\left(\boldsymbol{M}_{\mathrm{x}} \boldsymbol{p}\right)\right]=\mathbb{E}\left[\left(\boldsymbol{M}_{\mathrm{x}} \boldsymbol{p}\right)^{\prime}\left(\boldsymbol{M}_{\mathrm{x}} \boldsymbol{p}\right) \mathbb{E}\left(\boldsymbol{d} \mid \boldsymbol{M}_{\boldsymbol{x}} \boldsymbol{p}\right)\right]=c \mathbb{E}\left[\left(\boldsymbol{M}_{\mathrm{x}} \boldsymbol{p}\right)^{\prime}\left(\boldsymbol{M}_{\mathrm{x}} \boldsymbol{p}\right)\right]
$$

Assumptions B1 and B2 imply that:

$$
\mathbb{E}\left[\left(\boldsymbol{M}_{\mathrm{x}} \circ \boldsymbol{d}\right)^{\prime}\left(\boldsymbol{M}_{\mathrm{x}} \boldsymbol{\epsilon}\right)\right]=c \mathbb{E}\left[\left(\boldsymbol{M}_{\mathrm{x}} \boldsymbol{p}\right)^{\prime}\left(\boldsymbol{M}_{\mathrm{x}} \boldsymbol{\epsilon}\right)\right]
$$

Therefore, $\operatorname{plim}(\hat{\beta}-\beta)=0$ and the OLS estimate $\hat{\beta}$ is consistent. 


\section{Appendix D: Additional Analysis}

This section provides more details on several analyses that we do not have space for in the main paper. Section D.1 discusses how different cities are designiated into different rollout waves. Section D.2 uses sensitivity to precipitation to define consumption categories that are more 'outdoor' in nature. Sections D.3 and D.4 examine two private sources of pollution information and evalutes whether households engage in effective pollution avoidance prior to the monitoring program. Sections D.5 and D.6 conducts the IV and synthetic analyses of mortality effects. Section D.7 uses annual data to evaluate the robustness of avoidance findings.

\section{D.1 Determinants of Information Rollout Assignment}

Appendix Table E.3 reports an analysis on the determinants of the official rollout assignment. Columns (1)-(3) show a representation of Appendix Figure E.9 using three separate linear probability models. The models regress an indicator of a city being in wave $k$ in the rollout on three indicators for administrative hierarchies and pre-designated status that are denoted by the deep blue, light blue, or white group. ${ }^{51}$ Consistent with our discussion above, these simple models produce a very high $\mathrm{R}^{2}$ ranging from 0.65 to 0.84 .

We now examine the potential role of observed covariates in determining the official rollout timing. To make it simple, in column (4), we first estimate a benchmark model where the outcome variable is city's rollout wave as a continuous variable (take values 1 , 2 or 3 , and hence negative coefficients would mean earlier waves). Regressors include a constant term and indicators for deep blue and light blue cities; the indicator for white cities is the omitted category. This model is therefore a parsimonious summary of columns (1)-(3). Again, this simple model has a high $\mathrm{R}^{2}$ of 0.75 .

Column (5) bases off the specification of column (4), but asks whether observed covariates in the pre-policy period can provide additional explanatory power. Specifically, we examine pollution, income, and their trends. Pollution and pollution trends are measured by a city's 2006-2012 average AOD and its 2006-2012 annual change in AOD. Income and trends are measured by a city's 2006-2012 average per capita disposable income and its 2006-2012 annual changes in per capita income.

All covariates are normalized by dividing the mean, so the interpretation of the coefficients is "how much change in the rollout wave is associated with a doubling of the characteristics", with a negative coefficient meaning that an increase in the characteristics is associated with

\footnotetext{
${ }^{51}$ Note in column (3), the coefficient on light blue indicator is missing because there were no light blue cities in the wave-3 rollout. Also see Appendix Figure E.9.
} 
the city being in an earlier rollout.

Results in column (5) suggest cities with higher levels of pollution and higher levels of per capita income are significantly more likely to be in earlier waves. This is expected, as cities in earlier rollout waves tend to be more developed such as provincial capitals. ${ }^{52}$ We note that our analysis includes city fixed effects, and therefore any permanent differences in the levels of characteristics do not post a threat to the identification. On the other hand, trends in pollution and income prior to the monitoring program do not predict wave assignment. This suggests the rollout timing is not determined by changes in local conditions. Comparing $\mathrm{R}^{2}$ in columns (4) vs. (5), the additional covariates provide little improvement in explanatory power $\left(\mathrm{R}^{2}=0.747\right.$ in column (4) and $\mathrm{R}^{2}=0.769$ in column (5)).

Finally, column (6) presents a model with only the pollution and income covariates. Similar qualitative conclusions emerge: cities with high pollution and high income are in earlier waves but trends are, once again, not predictive. The model's goodness-of-fit is lower $\left(\mathrm{R}^{2}=0.427\right)$.

Overall, the regression analysis reinforces the view that the policy rollout is a top-down decision that reflects pre-existing administrative hierarchies and city characteristics rather than changes in local economic or pollution conditions.

\section{D.2 Outdoor Consumption Trips}

Our transaction data do not contain flags on the indoor/outdoor nature of the underlying purchases. To make progress, we consider a data-driven exercise that exploits heterogeneity in how precipitation affects transaction activities across 273 narrowly defined merchant category codes (MCCs). The underlying logic of this exercise is that merchant categories whose transaction volumes are more sensitive to precipitation events are more likely to be those that are either deferrable in nature or those that tend to occur in outdoor settings (and thus involve more pollution exposure). These are the types of transaction activities that we expect to exhibit more pronounced pollution avoidance behavior.

We begin by estimating the following regression separately for each of the 273 merchant categories. We use daily-level observations to detect responses to precipitation better.

$$
\log \left(\text { Transactions }_{m c t}\right)=\beta_{m} \cdot \text { Precipitation }_{c t}+\alpha_{m c}+\alpha_{m t}+\varepsilon_{m c t}
$$

where Transaction $_{m c t}$ is the number of transactions in merchant category $m$ in city $c$ on

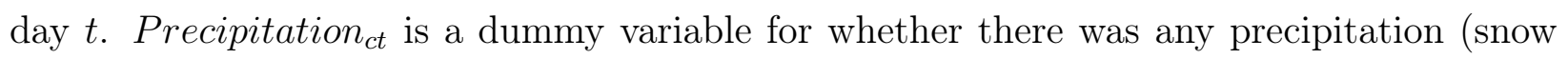
or rain) in the city-day. All regressions control for city fixed effects $\left(\alpha_{m c}\right)$ and week fixed

\footnotetext{
${ }^{52}$ We have shown similar quantitative patterns in Appendix Table E.4.
} 
effects $\left(\alpha_{m t}\right)$. We cluster standard errors at the city level. Panel (a) of Appendix Figure E.10 plots the estimates $\beta_{m}$ estimates. Each bar represents a merchant category $m$, and the range of each bar corresponds to the $95 \%$ confidence interval of the estimate. We rank merchant categories by their estimated sensitivity to precipitation, starting from those with the most negative point estimate of $\beta_{m}$. Blue bars highlight merchant categories whose $\beta_{m}$ estimates are statistically significant at the $5 \%$ level.

Two findings emerge from this exercise. First, the vast majority of precisely estimated effects have negative signs (i.e., precipitation reduces transactions). This is reassuring because virtually all transactions in our database occur in physical stores and necessarily involve outdoor trips, so we expect an overall negative association with precipitation. Second, at the bottom of the same chart, we use red bars to mark merchant categories in supermarkets, dining, and entertainment sectors that we defined as deferrable consumption trips in Table 2, Panel B of the paper. Our new analysis shows that these categories are disproportionately deferrable and/or outdoor in nature from a precipitation-sensitivity perspective. In Appendix Figure E.11, we tabulate the top 20 most precipitation-sensitive merchant categories according to our estimates. The most sensitive categories include snow car rentals and dealers, vacation houses, sports stadiums, garment stores, amusement parks, and optical exams and products, among others that are arguably deferrable in nature or occurring in open-air settings.

Next, we construct a new purchase frequency variable which equals the number of card transactions per 10,000 active cards in these top 20 merchant categories. Its mean is 56.3 transactions per week per 10,000 cards (for reference, the all-category transaction frequency mean is 869 transactions). We then repeat the shopping-pollution gradient analysis using this deferrable/outdoor purchase frequency as the dependent variable. Panel (b) of Appendix Figure E.10 reports the event study figure. The pollution gradient after the monitoring program exhibited a sharp drop of -3.12 transactions per 10,000 cards ( $\mathrm{SE}=1.27, p$-value $=$ $0.015)$, which represents a 5.5 percent reduction relative to the mean transaction frequency (56.3 per 10,000 cards). Recall from Table 2 that the effect size for all-category transactions is a 1.4 percent reduction. As expected, the effect size for the deferral categories is much more pronounced. This new analysis provides additional evidence that consumption trips that are more deferrable/outdoor in nature responded more strongly to pollution variations after the information became available. 


\section{D.3 Visibility as Alternative Pollution Information}

We assess the possibility that people used visibility as a proxy for pollution levels even before the pollution information program. We obtain weather-station-based daily visibility data covering all cities from China's National Meteorological Administration for 2011-2016. Panel (a) of Appendix Figure E.12 plots the distribution of the raw visibility measure. For the vast majority of stations, visibility is top coded at $30 \mathrm{~km}$ (18.6 miles).

How accurate is visibility as a proxy for air pollution? Panel (b) of Appendix Figure E.12 shows the median, inter-quartile range, and 10-90 percentile range of satellite-based AOD by ventile bins of visibility. Lower visibility bins (the left part of the x-axis) mean poor visibility conditions. We find an overall downward-sloping relationship between pollution and visibility, which suggests that visibility provides useful information on air quality, especially when visibility is high. However, the relationship features substantial uncertainty when visibility is poor. For example, for the bottom $20 \%$ days with the lowest visibility, the 10-90 quantile range of $\mathrm{AOD}$ can vary from 0.35 (decent air quality, compared to a national average of 0.55 ) to 1.55 (poor air quality). These patterns underline the challenges that people face in trying to avoid pollution without actual pollution monitoring data: both heavy fog and heavy smog could cause poor visibility outdoors. Therefore, the scope for using visibility as a proxy for pollution is limited.

It is nevertheless interesting to empirically test whether residents used visibility as a proxy for pollution and exercised avoidance, and how that relationship interacts with actual pollution variation. To operationalize the test, consider the following two sets of estimation equations, where we use the "pre, post" superscripts to note that we estimate coefficients of interest for both before and after the monitoring program:

$$
\begin{gathered}
\text { Transactions }_{c t}=\Gamma^{\text {pre,post }} \cdot \text { Visibility }_{c t}+\alpha_{c}+\alpha_{t}+\varepsilon_{c t} ; \\
\text { Transactions }_{c t}=\gamma^{\text {pre,post }} \cdot \text { Visibility }_{c t}+\beta^{\text {pre,post }} \cdot \text { Pollution }_{c t}+\alpha_{c}+\alpha_{t}+e_{c t} .
\end{gathered}
$$

We leverage these two sets of estimation equations to answer the following four questions:

Question 1. Did purchase trips respond to outdoor visibility before pollution information was available $\left(\Gamma^{\text {pre }}\right)$ ?

Question 2. Did the purchase-visibility relationship change after pollution monitoring began $\left(\Gamma^{\text {pre }}\right.$ vs. $\left.\Gamma^{\text {post }}\right)$ ?

Question 3. If the answer to Question 2 is yes, then how much of that change was explained by people's response to "real" pollution ( $\Gamma^{\text {pre,post }}$ vs. $\left.\gamma^{\text {pre,post }}\right)$ ? 
Question 4. Once we take into account that people may use visibility as a proxy for pollution, does "real" pollution variation still matter for purchase trips $\left(\beta^{\text {pre,post }}\right)$ ?

Panels (c) and (d) of Appendix Figure E.12 summarize the answers to Questions 1, 2, and 3. The blue lines show the estimates for $\Gamma^{\text {pre,post }}$, and the orange lines show the estimates for $\gamma^{\text {pre,post }}$.

Before the monitoring program, we observed fewer card transactions during weeks when visibility is in the lowest quintile. However, the shopping-visibility relationship is nonmonotone, economically modest, and imprecisely estimated. The reduction in card transactions during weeks with the lowest visibility may reflect a genuine concern for poor visibility per se (e.g., road safety concerns) instead of pollution. Overall, the data provide little support that people used visibility as a proxy for pollution and engaged in avoidance before pollution monitoring.

The blue line in Panel (d) of Appendix Figure E.12 shows that the negative relationship between visibility and card transactions became stronger after monitoring, which is consistent with our main findings based on the pollution measure.

More importantly, adding pollution controls barely changes the transactions-visibility relationship before the monitoring program (the orange line is very close to the blue line). If people were using visibility to predict pollution levels, the transactions-visibility relationship should change after we add pollution controls. This is indeed the pattern we find post the monitoring program: controlling for pollution attenuates the transactions-visibility relationship, especially at low visibility ranges.

To numerically summarize our findings, Appendix Table E.9 reports specifications where the key regressors are log-inverse visibility "Log(1/Visibility)" (higher values stand for lower visibility), pollution "Log(Pollution)", and their interactions with the post-monitoring dummy.

Columns (1) through (4) examine the relationship between card transactions and poor visibility and how the relationship changes before and after the monitoring policy. There is no evidence that people responded to visibility before the monitoring program (the coefficient for poor visibility, $\log (1 /$ Visibility), is mostly imprecisely estimated and positive). The interaction coefficient is negative, suggesting that people were more responsive to poor visibility after the monitoring program. However, unlike the case for pollution (Table 2 in the paper), the interaction coefficients are not precisely estimated.

Columns (5) through (8) show that, once pollution and its interaction with the postinformation dummy are included in the specifications, the gradient change in (1/Visibility) is largely explained away (and of wrong sign in Columns (7) and (8)). On the other hand, the visibility terms cannot explain away the pollution findings. The "Log(Pollution) $\times 1$ (after monitoring)" coefficients from columns (5) through (8) ( $\beta^{\text {pre,post }}$ in Question 4 above) are 
similar in magnitude to those from our preferred specifications where we only looked at the effect of pollution (Table 2 of the paper), though more noisily estimated when both the visibility and pollution terms are included as regressors.

Overall, the data do not support the conjecture that consumers engaged in effective pollution avoidance through visibility cues likely due to: (1) visibility is a noise indicator of pollution when visibility is low (e.g., it could be due to fog), and (2) the general public had limited awareness of air pollution before the program.

\section{D.4 U.S. Embassy $\mathrm{PM}_{2.5}$ Data in Beijing as Alternative Pollution Information}

We consider the possibility that independent pollution monitoring conducted by the U.S. Embassy in China might have been used as an alternative pollution measure. Five cities in China with U.S. Embassy and Consulates (Beijing, Chengdu, Guangzhou, Shanghai, and Shenyang) operate independent $\mathrm{PM}_{2.5}$ monitoring. Our analysis focuses on Beijing, the only city with $\mathrm{PM}_{2.5}$ data going back to January 2011 that allows our regression analysis to include seasonal controls before and after the monitoring program. Appendix Figure E.13 summarizes the results. We examine web searches for smog in panel (a) and card transactions in panel (b). For each outcome, we show a decile bin scatterpolot of the relationship between the outcome and Embassy $\mathrm{PM}_{2.5}$ and do so separately for the pre-policy (i.e., 2011 and 2012) and post-policy (i.e., 2013-2016) periods. The difference in the slope of the two fitted lines (gray for pre-policy, blue for post-policy) corresponds to what we call "change in pollution gradient" - that is, the change in how the outcome variable responds to pollution, before vs. after information availability. With weekly time series data from one city we cannot have detailed control variables. For both the pre- and post-policy periods, we residualize each outcome with 52 week-of-year indicators to parse out seasonality. The analysis contains 312 observations.

Panel (a) in Appendix Figure E.13 shows that the correlation between web searches and pollution readings was close to zero before 2013. It is a tight 'zero' relationship as the dots lie close to the linear fitted line. This suggests that the U.S. Embassy $\mathrm{PM}_{2.5}$ monitoring data, which were reported on Twitter since 2008, were not a significant information source for the average resident in Beijing. If they were, Beijing residents would have responded to such information before 2013. After 2013, the search-pollution gradient became much more positively correlated, with searches going up substantially on weeks when $\mathrm{PM}_{2.5}$ concentration exceeds $100 \mu \mathrm{g} / \mathrm{m}^{3}$.

Panel (b) shows that the correlation between U.S. Embassy pollution readings and weekly 
card transactions was also flat before 2013. After 2013 when pollution information became available, the correlation became significantly negative, and doubling the pollution level is associated with 195 fewer transactions per 10,000 active cards per week. The magnitude of this estimate is much larger than our full-sample estimate (about 12 fewer transactions per 10,000 active cards per week). This is partly because Beijing has a higher baseline transaction rate (1,232 weekly transactions per 10,000 cards compared to a national average of 869) and because the levels of pollution in Beijing tend to be higher (daily mean $=79$ $\mu \mathrm{g} / \mathrm{m}^{3}, \mathrm{IQR}=29$ to $109 \mu \mathrm{g} / \mathrm{m}^{3}$ in 2015 , compared to the national average daily mean $=39$ $\mu \mathrm{g} / \mathrm{m}^{3}, \mathrm{IQR}=24$ to $\left.63 \mu \mathrm{g} / \mathrm{m}^{3}\right)$.

\section{D.5 Instrumental Variables Approach}

Our empirical analysis shows that the monitoring program has reduced the mortality-pollution gradient, i.e., the mortality impact of air pollution was mitigated after the program relative to that before the program likely due to behavioral changes as documented in our analysis. While the impact of pollution on mortality itself is not the focus of our paper (we evaluate its changes), it might be of interest to policymakers. In this section, we recover the causal estimate of the pollution's mortality effect using IVs to address the endogeneity in pollution.

We implement a wind-transport instrumental variable (IV) approach in the spirit of Bayer, Keohane and Timmins (2009), Deryugina et al. (2019), Anderson (2020) and especially Barwick et al. (2020). The goal is to tease out (exogenous) variation in a city's local air pollution attributable to transported pollutants from upwind cities. Our IV is essentially a function of wind directions, wind speed, weather conditions, and distance of origin and destination cities. To see the IV construction, we use the city of Beijing as a narrative example. We begin with a daily panel dataset of $\mathrm{PM}_{2.5}$ in a set of cities whose pollution level may affect air quality in Beijing. Let $C$ denote the set of contributing cities. For each city $c$ and day $t$, we calculate the radian angle $\phi_{c t}$ between city $c$ 's local wind direction and the vector pointing from city $c$ to Beijing (e.g., $\phi_{c t}=0$ if city $c$ is exactly upwind from Beijing on day $t$ ). The IV is a time-series variable constructed using the following formula:

$$
\mathrm{IV}_{t}=\sum_{c \in C} \max \left\{0, \cos \left(\phi_{c t}\right)\right\} \cdot \text { Pollution }_{c t} \cdot\left(\frac{1 / \text { distance }_{c}}{\sum_{i \in C} 1 / \text { distance }_{i}}\right)
$$

where the term $\max \left\{0, \cos \left(\phi_{c t}\right)\right\} \cdot$ Pollution $_{c t}$ - which we call "upwind pollution" - is the vector component of air pollution in city $c$ on day $t$ that is expected to move toward Beijing. We assume upwind pollution is zero if $\phi_{c t}$ is an obtuse angle, i.e., winds in city $c$ on day $t$ are blowing away from the direction toward Beijing. On any date $t$, the IV is the average of 
individual cities' upwind pollution terms, inversely weighted by city c's distance to Beijing (distance dis. .

The choice of contributing cities (denoted as $C$ ) for our analysis involves a trade-off between bias and variance. Using cities that are very far from Beijing helps the exclusion restriction assumption of the IV (that is, the assumption that transported pollution from distant cities does not affect mortality in Beijing, except through its impact on local air quality). However, focusing on pollution from cities too far away hurts the first stage assumption because the local impact of upwind pollution from faraway cities is weak. We take the following steps to address such bias-variance trade-off. First, we restrict contributing cities to those that are at least $300 \mathrm{~km}$ away from Beijing. ${ }^{53}$ Second, among the remaining cities, we employ a data-driven method that selects the most predictive upwind cities in a "zero-stage" Lasso regression. Specifically, before constructing the IV variable, we estimate the following equation with linear Lasso:

$$
\text { Pollution }_{\text {Beijing }, t}=\lambda_{0}+\sum_{c \in \tilde{C}} \lambda_{c} \cdot \max \left\{0, \cos \left(\phi_{c t}\right)\right\} \cdot \text { Pollution }_{c t}+\epsilon_{t},
$$

which selects a subset of 73 upwind cities from a total of 330 cities that are at least 300 $\mathrm{km}$ away from Beijing. Appendix Figure E.16 maps out the location of these upwind cities and their corresponding $\lambda_{c}$ coefficients in the post-Lasso regression. We then construct the IV variable for Beijing using these selected cities as outlined in Equation (D.6). We repeat this procedure for all cities in our sample. The first stage of our IV is strong, with the Kleibergen-Paap F-statistic exceeding 600.

Having constructed the IV, we use 2SLS to estimate the mortality impact of pollution separately for the pre and post periods following Equation 1: We include city, week-of-year, and year FEs, as well as city-specific trends, as in the main analysis. Standard errors are clustered at the city level. Appendix Figure E.17 reports the parameter estimates capturing the mortality impact of pollution before and after the monitoring program, separately by the cause of deaths. Panel (a) shows the OLS results while Panel (b) shows the IV results.

Several patterns emerge from this analysis. First, our IV estimates are in general larger in magnitude than OLS estimates, consistent with the literature (Deschenes and Greenstone, 2011; Ebenstein et al., 2017; Deryugina et al., 2019) that OLS estimates of the health impact of air pollution are small and often insignificant. For example, before the monitoring program, doubling pollution increased the all-cause mortality rate (the first blue solid dot in both figures) by $0.64 \%$ according to the OLS regression (Panel (a)) and $2.35 \%$ according to

\footnotetext{
${ }^{53}$ Similar strategies have been used in the prior literature. For example, Williams and Phaneuf (2019) uses a buffer zone of 60-120 km radius; Barwick et al. (2020) uses a buffer zone of $150 \mathrm{~km}$ radius.
} 
the IV regression (Panel (b)). The magnitude of the IV estimates is intuitive: the mortalitypollution elasticity is highest for the respiratory category at $6.74 \%$ (as $\mathrm{PM}_{2.5}$ directly affects the human respiratory system). The pollution elasticity is close to zero for injuries, which is also sensible.

Second, for both IV and OLS estimates, the monitoring program is associated with a reduction in the pollution elasticity, i.e., a decrease in the pollution gradient. In the figure, the hollow dots (which denote post-monitoring estimates) shift downward from the solid dots (which denote pre-monitoring estimates) for all of the cause categories except for injuries. That is, post the monitoring program, the pollution's mortality damage is partially mitigated, consistent with our main estimates on the change-in-gradient shown in Table 3.

Third, for all-cause mortality, the change in coefficients is virtually the same whether we use OLS or IV regressions (at 0.02 log points). The orange dashed lines in both figures provide a visual aid to this result, with the two lines having the same length and slope. This finding provides support to the identification assumption in our change-in-gradient analysis: the magnitude of bias appears similar before vs. after the monitoring program and hence cancels out when we focus on the change in the gradient itself.

\section{D.6 Synthetic Control Analysis}

The key step of this exercise is that, for each city, we need to identify a set of "control" cities that can serve as reasonable counterfactuals in the absence of the monitoring program. To identify these control cities, we use the synthetic control method following Abadie, Diamond and Hainmueller (2010) and Arkhangelsky et al. (2021). For each city $\tilde{c}$, we create a group $g$ which contains the city itself and all of its control cities: $g=\left\{\tilde{c}, c_{1}, \ldots, c_{k}\right\}$ with associated synthetic weights $w=\left\{1, w_{1}, \ldots, w_{k}\right\}$ such that $\sum_{i=1}^{k} w_{i}=1$. We will henceforth call $\tilde{c}$ the "treated" city and $\left\{c_{1}, \ldots, c_{k}\right\}$ the "control" cities.

We make two decisions regarding the choice of the control cities and the synthetic weights. First, each treated city is matched to control cities that have not yet been treated by the monitoring program. That is, for each city in wave 1 , we choose control cities from waves 2 and 3 ; for each city in wave 2 , we choose controls from wave 3 cities. We drop wave 3 cities because they are in the last wave of the information rollout and there are no untreated cities to serve as their controls.

Second, we select control cities based on outcomes prior to the monitoring policy, but compute synthetic weights using data up to one year before the information program is actually implemented. This allows us to use the year prior to the pre-treatment period as a validation sample to examine the synthetic control method's performance. 
We estimate the following difference-in-differences estimation equation:

$$
\begin{aligned}
\log \left({\text { Mortality })_{g c t}=}\right. & \beta \cdot \text { Treated }_{g c} \times \text { Post }_{g t}+\gamma \cdot \text { Treated }_{g c} \\
& +\theta \cdot \text { Post }_{g t}+\boldsymbol{x}_{c t}^{\prime} \boldsymbol{\theta}+\varepsilon_{c t},
\end{aligned}
$$

where $g$ denotes a group that contains a treated city and its control cities. A unit in this panel-data regression is a city $c$ in a group $g$ (thus the subscript $g c$ ). Treated Tr $_{c}$ is a dummy variable for the treated city in each group. Post $t_{g t}$ indicates post-treatment periods for group $g$ based on the treated city's treatment time. The regression is weighted by group-by-city level synthetic weights and includes city-by-group, week-of-year, and year fixed effects, as well as city-by-group-specific trends. Standard errors are clustered at the city level.

Panel (a) in Appendix Figure E.18 shows the event study results. The blue line at the bottom of the chart represents the difference between treated cities' mortality rates and their synthetically weighted control cities' mortality rates as a function of time relative to the information rollout. All periods prior to eight months before the monitoring policy are pooled into one group and used as the reference group. The regression includes city, weekof-year, and year fixed effects. The orange line on the top shows the average pollution level in the corresponding event time. The shaded areas highlight the quarters when a pollution peak occurred.

Several patterns emerge from the graph. First, the blue stays close to zero during the matching period. This means that, as expected, treated and control cities' mortality rates track each other closely during the period used to compute synthetic weights. Second, during the validation period (i.e., one year prior to the information rollout), mortality rates in the treated and control cities continue to be close to each other. Our estimate suggests that, relatively to the the matching period, mortality rate decreases by 0.25 percent $(\mathrm{SE}=1.16$ percent) during the validation period. Third, we estimate that the mortality rate decreases by 1.64 percent on average after the monitoring policy. This estimate is imprecise with $\mathrm{SE}=1.70$. However, comparing the blue (mortality) and the orange (pollution) lines, we notice much more pronounced gaps in mortality rates between the treated city and the control group during heavily polluted periods post the information program, a visual pattern that was not apparent before the monitoring rollout. In contrast, differences in mortality levels during low pollution periods are modest before vs. after the program. These qualitative patterns suggest that the imprecise average effect of the information program may be masked by important heterogeneity.

To formally analyze this possibility, we test if the reduction in mortality rate after the monitoring program concentrates on periods when the city experiences high pollution lev- 
els. We group each city's weekly observations into quintiles based on its weekly pollution concentration. Then we estimate an augmented version of Equation (D.8), allowing the $\beta$ coefficient to vary by the pollution quintiles.

Panel (b) in Appendix Figure E.18 reports the heterogeneous $\beta$ coefficients. Each coefficient represents a synthetic difference-in-differences estimate regarding the monitoring program's impact on mortality within a quintile of a city's pollution levels. The monitoring program has no discernible impact on the mortality rate during periods of low pollution levels, but reduced mortality significantly (both statistically and economically) during high pollution periods. For example, the mortality rate decreases by more than four percent in weeks with the 20 percent highest pollution levels after the monitoring program. Importantly, this result is "isomorphic" to our gradient analysis in the main text of the paper, where we document a negative coefficient for the pollution and post-treatment interaction. In theory, this negative relationship could be driven by an increase in mortality during low pollution periods, a decrease in mortality during high pollution periods, or some combination of both. The new insight from the quintile analysis above shows that our main finding is mostly driven by a net reduction in mortality during high pollution periods post the monitoring program. The findings provide empirical justificatioon of our focus on the gradient analysis which leverages the fact that the mortality impact of the program is critically through its interaction with pollution. That is, rather than a (uniform) level shift in the mortality rate across different pollution levels, the program resulted in a change in the slope of the mortality-pollution relationship.

\section{D.7 Annual Analysis}

We conclude the empirical exercise with a robustness analysis that uses annual pollution measures. Specifically, we use the annual $\mathrm{PM}_{2.5}$ modeling data from Van Donkelaar et al. (2016), which combines satellite-based AOD and chemical transport modeling tools to provide estimates of ground-level $\mathrm{PM}_{2.5}$ concentration at the $10 \mathrm{~km}$-grid-by-annual frequency. We use this data to calculate two versions of city-year level $\mathrm{PM}_{2.5}$. The first version uses the 10 -by-10km grids that correspond to the ground locations of the pollution monitors and averages over these readings to get a city average. This is closest to the level of air quality that would have been captured by the monitors. The second version averages the pollution information from all grids within the city border, which allows us to measure overall pollution conditions in the city. The annual-level estimation model mirrors the primary specification Equation (1), with a few minor differences.

Given the annual frequency, we assign years 2013-2016 as the post period for wave one 
cities, 2014-2016 for wave two cities, and 2015-2016 for wave three cities. The regression includes city and year fixed effects $\left(\eta_{t}\right)$ and clusters standard errors at the city level.

Appendix Table E.10 displays the results. Each panel-column represents a regression. The panels differ by the type of pollution measures used: Panel A uses modeling $\mathrm{PM}_{2.5}$ near pollution monitors and Panel $\mathrm{B}$ uses modeling $\mathrm{PM}_{2.5}$ citywide. For reference, panel $\mathrm{C}$ uses the satellite-based AOD as in the paper but aggregates to the annual level. Column (1) reports results for card transactions. The outcome variable is annual rather than weekly transaction rates, and therefore the magnitudes of the coefficients are much larger than the weekly coefficients in Table 2 of the paper. Consistent with our original analysis, the coefficient of "Log(Pollution) $\times 1$ (after monitoring)" shows a negative sign regardless of which pollution measure we use. The magnitude is smaller than if one were to linearly scale up the weekly coefficient from Table 2 of the paper to the annual level (-12 transactions per week 52 weeks). This is consistent with intertemporal substitution of consumption. For example, a consumer might postpone a shopping trip due to high pollution, but the trip would eventually occur at some future point.

Column (2) reports mortality results. Once again, the coefficients are consistently negative, which echoes our findings in the paper using weekly data. In terms of the magnitude, we find relatively larger effect estimates at the annual level than those from the weekly analysis. This reflects the fact that the mortality effect of pollution in a given week takes more than one week to fully manifest, which is consistent with the findings in the literature (e.g., (Deryugina et al., 2019)). With the much smaller sample size, however, the annual estimates are imprecise in all three panels.

We take away from these exercises that the annual level analysis using alternative pollution measures gives us a broadly consistent conclusion, but we are underpowered by a 50-fold reduction in sample size. The annual level analysis discards rich, short-term pollution variations that are useful to identify the impact of the monitoring program on individuals' avoidance and health responses. In addition, there is evidence that the modeling $\mathrm{PM}_{2.5}$ measure might not provide accurate estimates of ground-level pollution in countries where pollution levels are high (Greenstone et al., 2021; Fowlie, Rubin and Walker, 2019).54

\footnotetext{
${ }^{54}$ Greenstone et al. (2021) calculate trends in $\mathrm{PM}_{2.5}$ pollution in China since 2013 and find that the values base on modeling data are lower than the levels recorded by the government monitors. A similar pattern is documented in Fowlie, Rubin and Walker (2019) using the U.S. portion of the modeling PM 2.5 data. They find that the modeling data understate $\mathrm{PM}_{2.5}$ at levels above $15 \mu \mathrm{g} / \mathrm{m}^{3}$.
} 


\section{Appendix E: Figures and Tables}

Figure E.1: Screenshot of China's Air Quality Disclosure Platform Web Interface

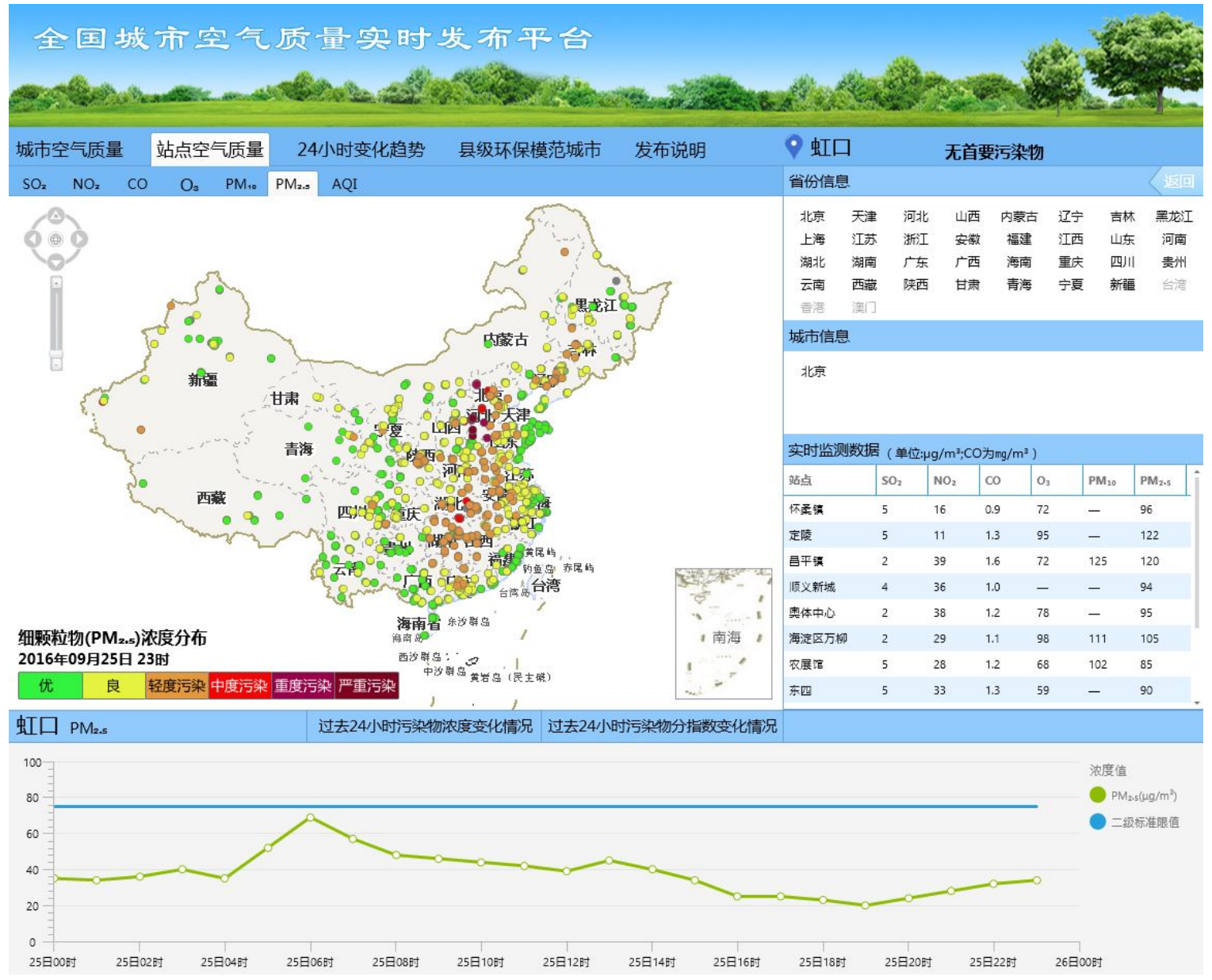

Notes: This figure shows a screenshot of the Ministry of Environmental Protection's real-time air quality disclosure platform web interface as of September 25, 2016. The left panel is an interactive map that displays the locations of all monitoring stations. The right panel reports real-time measures of six major pollutants for all monitoring stations in the city that is specified (Beijing). 
Figure E.2: Screenshot of an Air Quality App
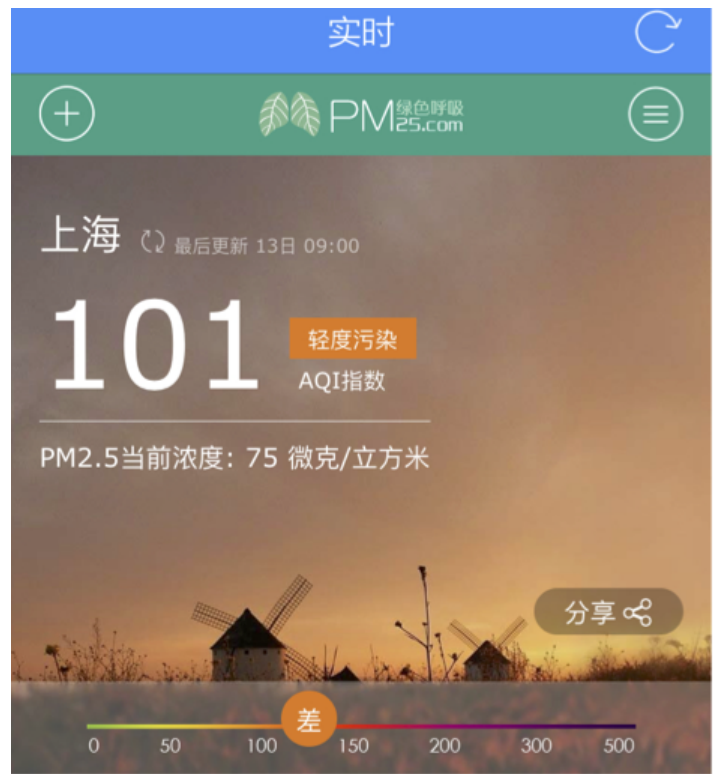

上海今日

预计24小时内PM2.5吸入量为 1091.2 微克, 沉淀进肺 泡无法排出量为 727.5 微克, 导致心肺疾病死亡风险升 高7.8\%, 癌症死亡风险升高 $11.2 \%$

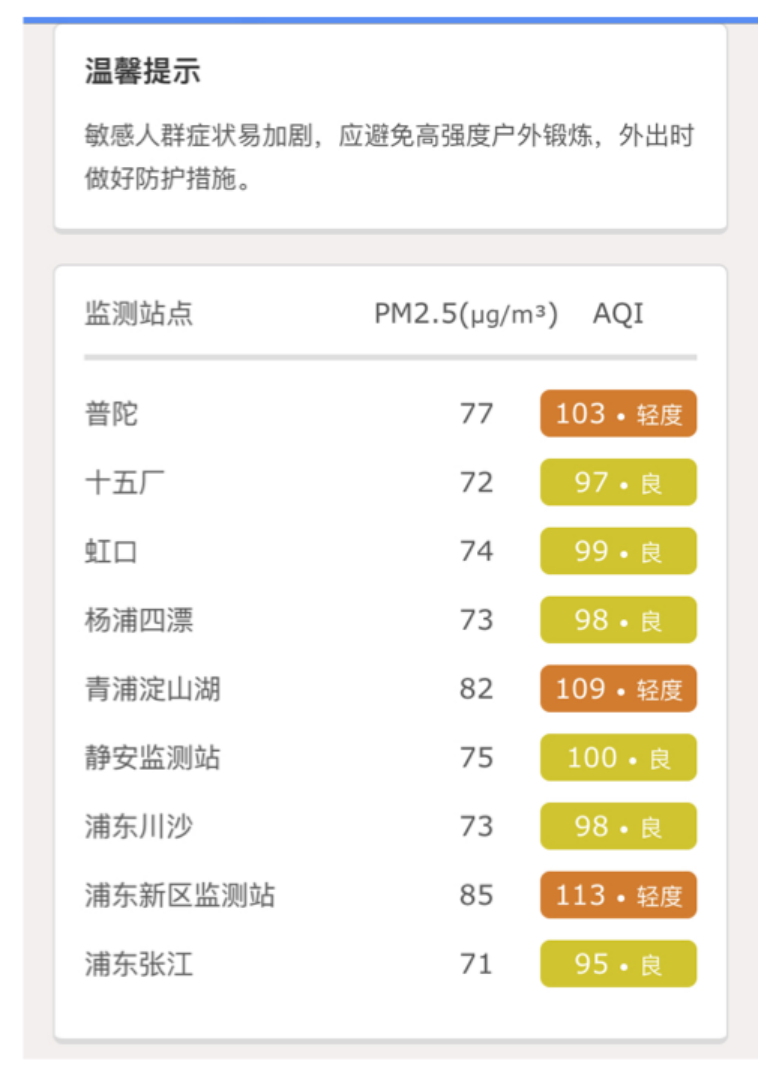

Notes: This figure shows a screenshot of a typical air quality app. The left panel shows the air quality index (AQI) in the city of Shanghai for that hour is 101 and $\mathrm{PM}_{2.5}$ is $75 \mathrm{ug} / \mathrm{m} 3$. The right panel shows $\mathrm{PM}_{2.5}$ and AQI readings at different monitoring stations in Shanghai. 
Figure E.3: Consumption Trends: UnionPay vs. National Accounts

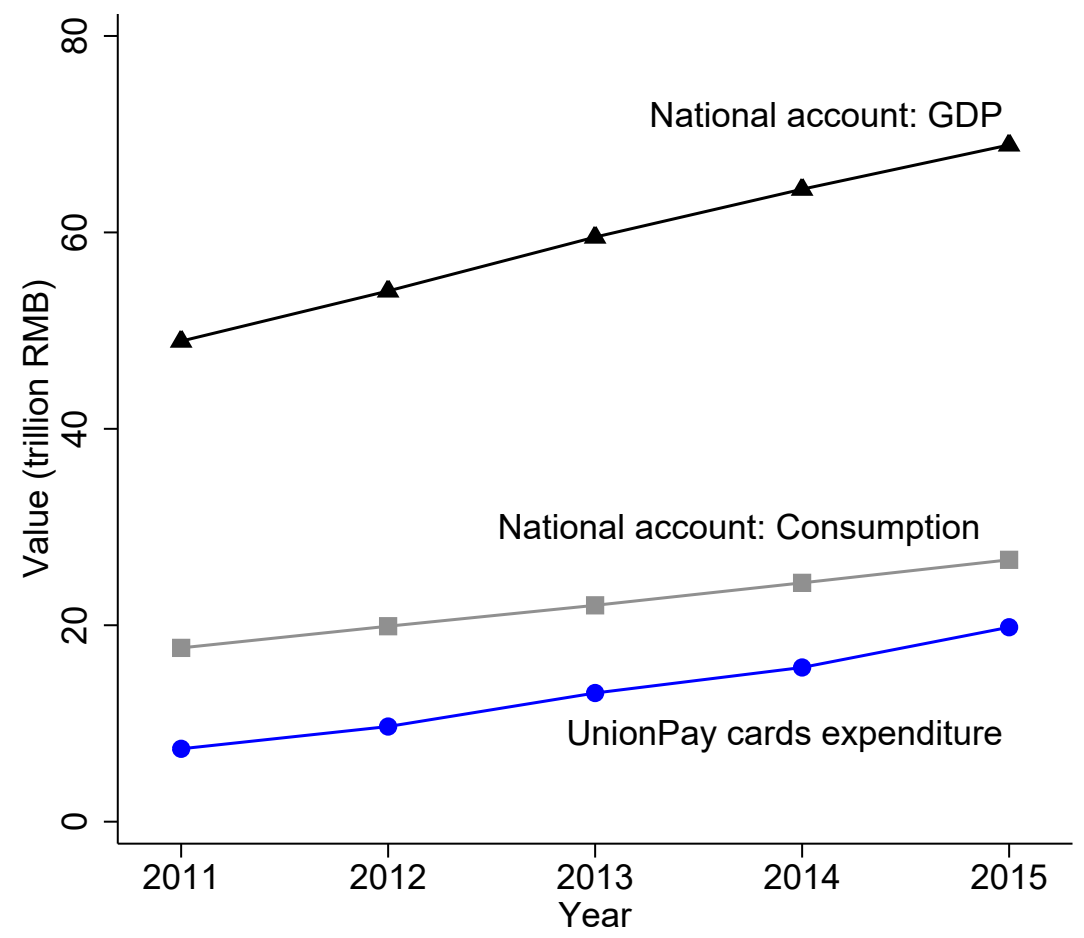

Notes: This figure plots annual GDP (triangles) and aggregate consumption (squares) reported by the National Bureau of Statistics of China (NBS), and total bank card spendings $\times 100$ (circles) aggregated from the UnionPay 1\% bank card data, excluding business to business transactions (the wholesale category). 
Figure E.4: UnionPay Bank Card Penetration by City, 2011-2015 Average

(a) Number of active cards

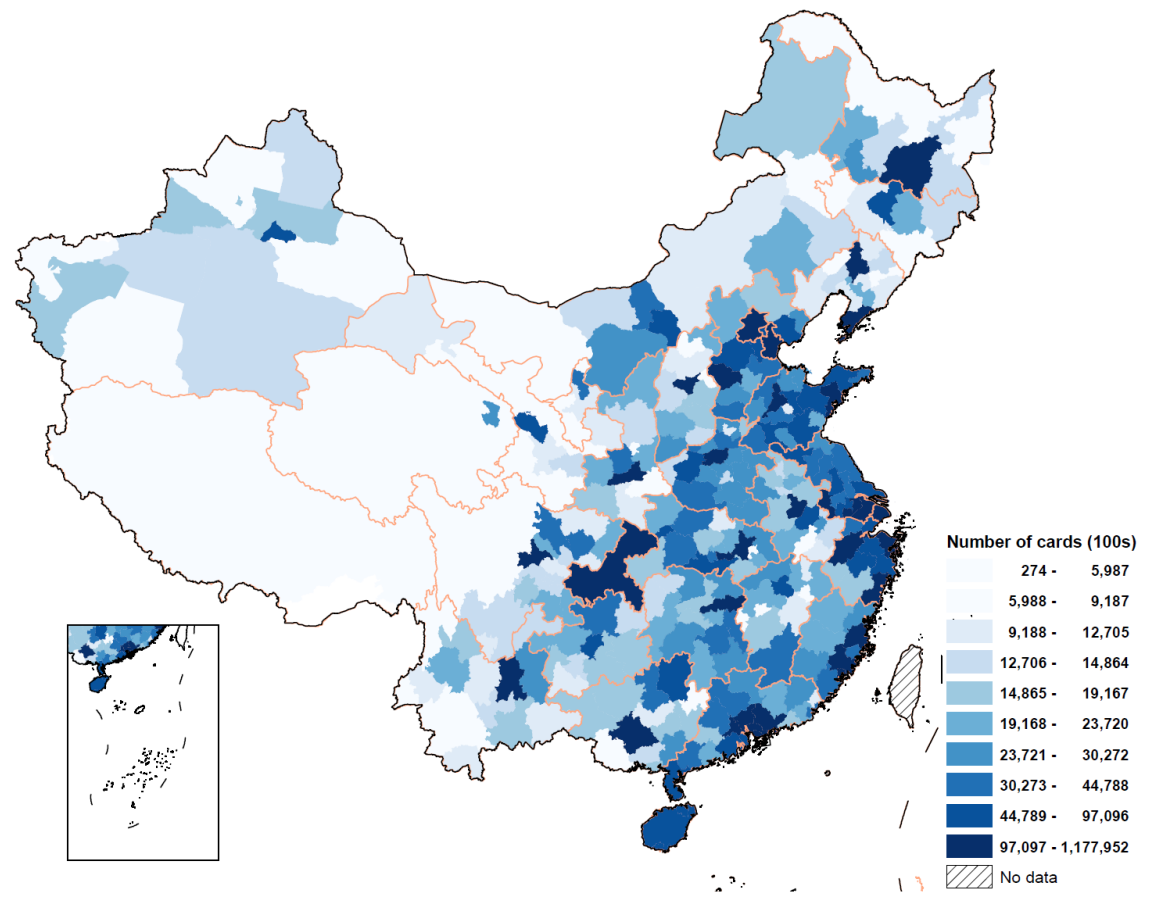

(b) Number of daily transactions per 100,000 cards

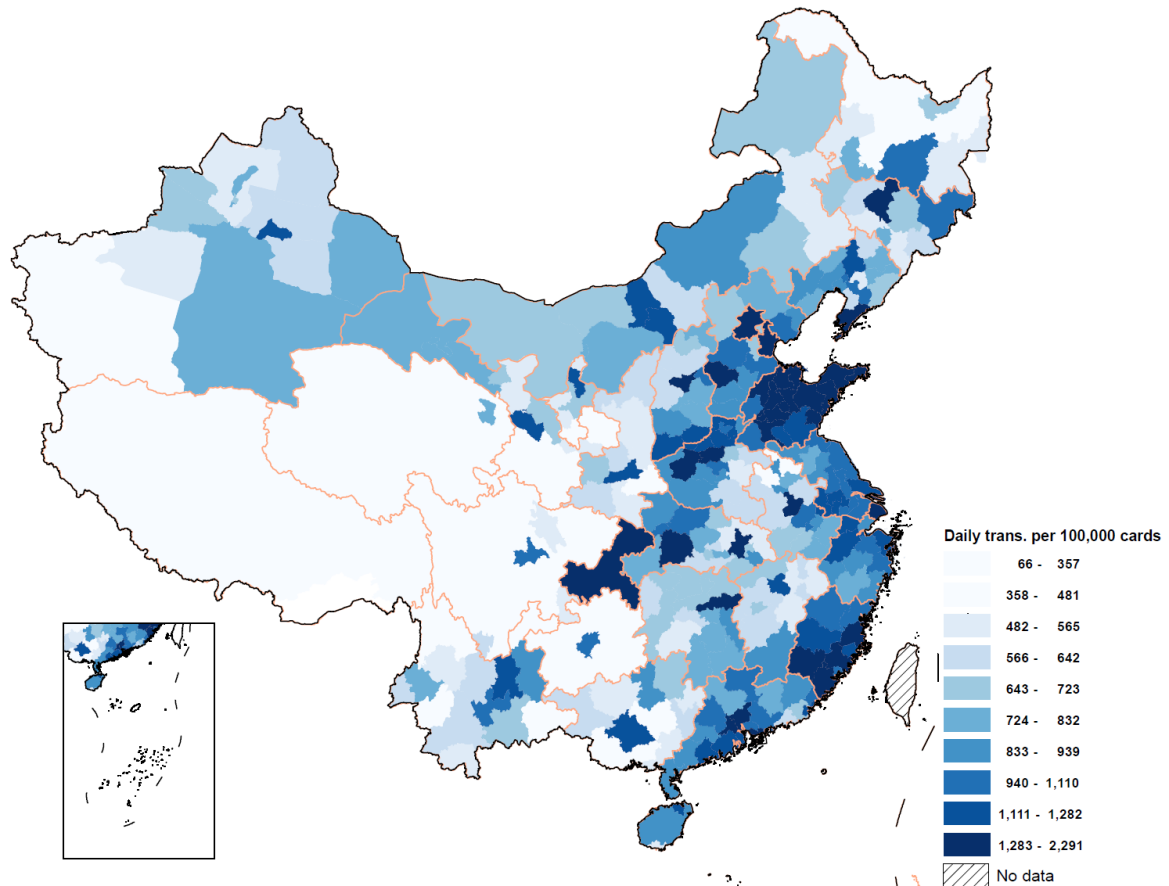

Notes: The maps show the 2011-2015 average number of active UnionPay bank cards (panel (a)) and daily transactions per 100,000 cards (panel (b)) at the city level. Orange lines show inter-provincial borders. 
Figure E.5: Correlation between $\mathrm{PM}_{2.5}$ and AOD

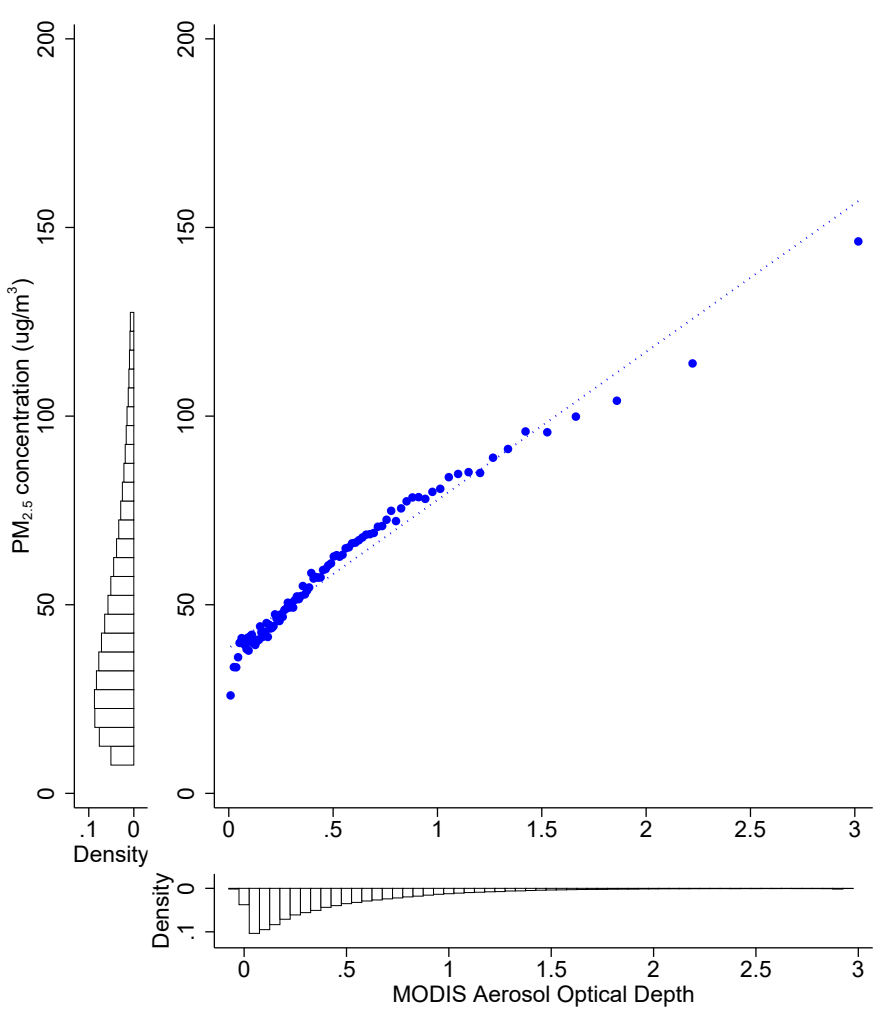

Notes: This graph shows city $\times$ day level average $\mathrm{PM}_{2.5}$ concentration (y-axis) by 100 equal bins of AOD (x-axis), for periods after the monitoring program. There is no reliable information on $\mathrm{PM}_{2.5}$ before the program. Histograms show the distribution of the two variables. 


\section{Figure E.6: Event Time Plots with Different Controls}

(a) News mentions

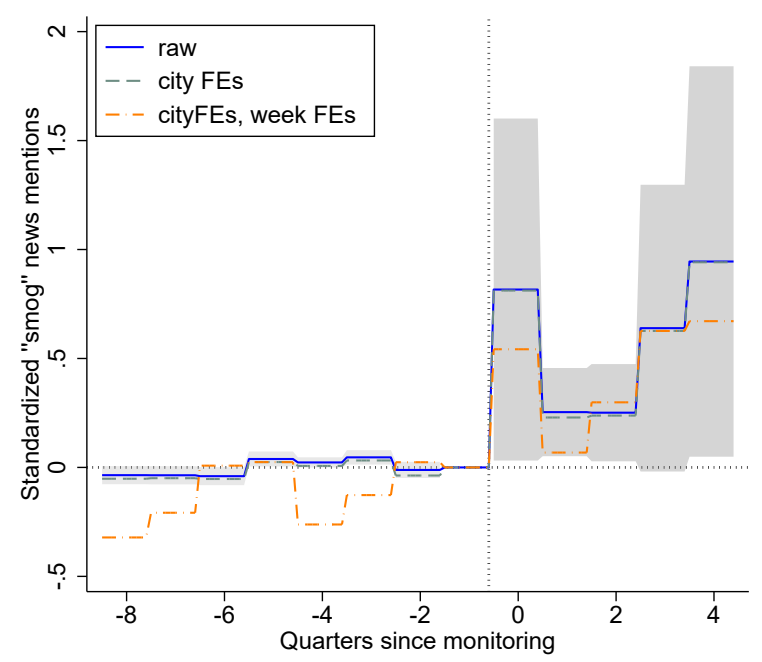

(b) Web searches

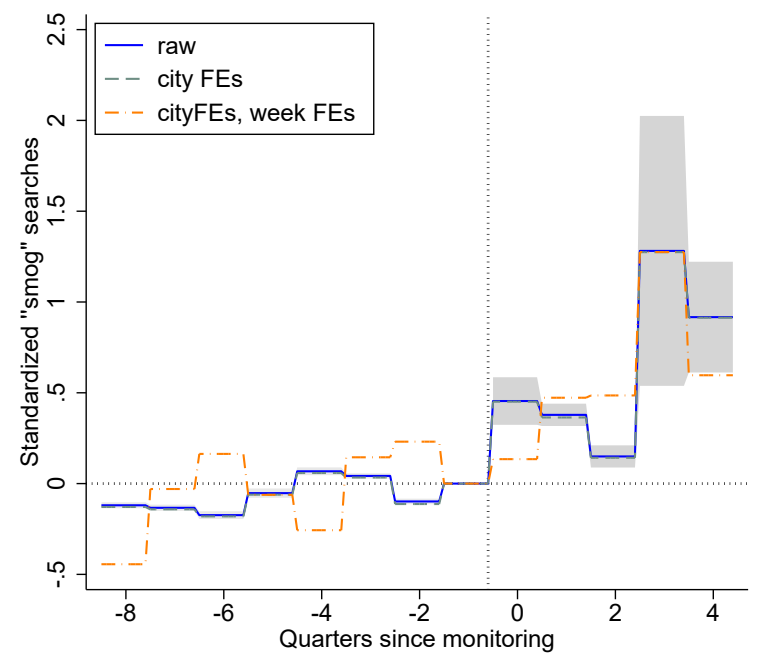

(c) Air purifier purchases

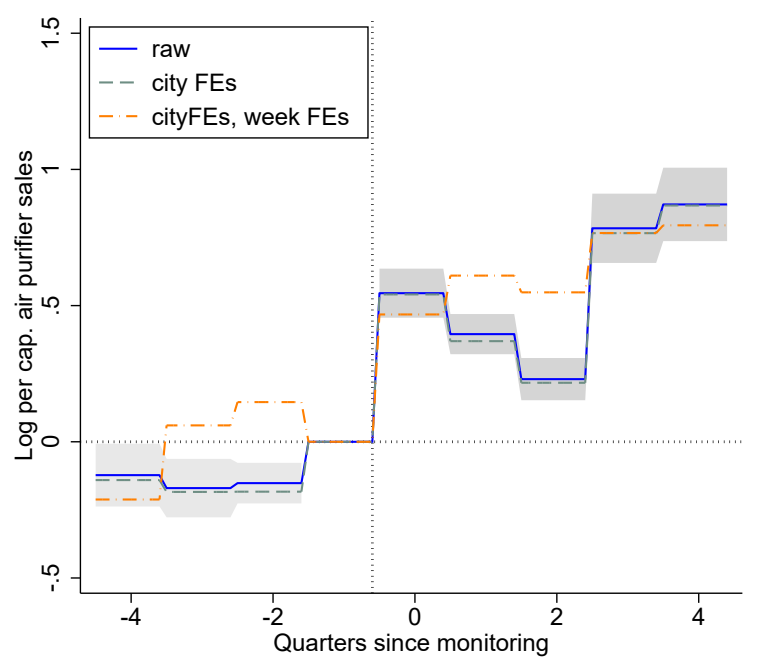

Notes: Figures plot standardized news mentions for smog (panel (a)), standardized smog-related web searches (panel (b)), and log per-capita air purifier purchases in large 50 cities (panel (c)) as a function of quarters since the completion of the monitoring program in a given city. Event quarter -1 is normalized to 0 . Solid blue lines show raw data patterns, green dashed lines control for city fixed effects, and orange dashed lines control for city and week fixed effects. Shaded regions show the $95 \%$ confidence intervals for the raw data pattern (the blue lines) and are constructed from standard errors clustered at the city level. 
Figure E.7: Changes in Pollution Information Access - Mobile Phone Apps

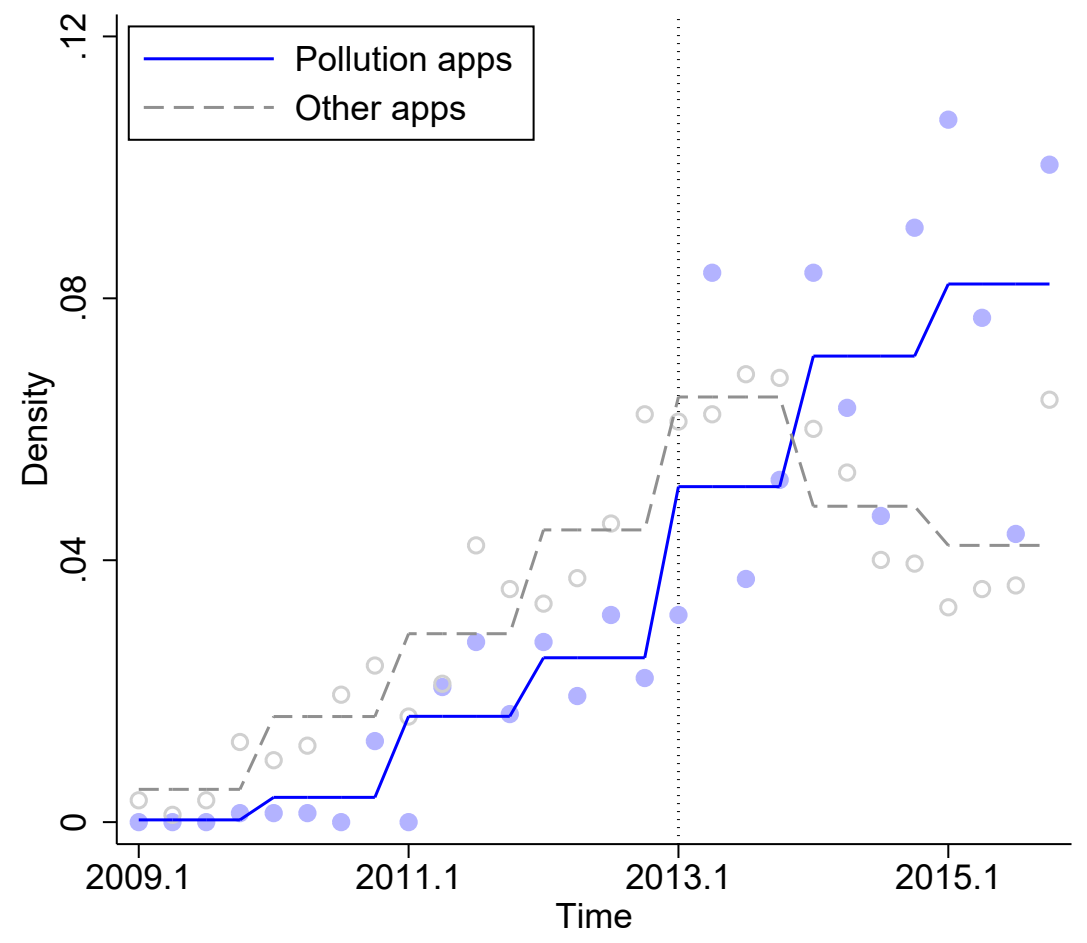

Notes: This chart shows the release-date distribution of Apple App Store apps separately for pollution apps (solid dots and line) and other apps (hollow dots and dashed line). Apps in other categories include games, music, video, reading, finance, sports, education, shopping, and navigation. For each category, sample is restricted to the first 200 apps returned by the Apple API given the search key. Data are queried from Apple App Store on December 27, 2015. Pollution apps released before 2013 typically stream weather information and incorporate real-time air quality content post 2013. 
Figure E.8: Citizen Complaints on Air and Water Pollution Issues

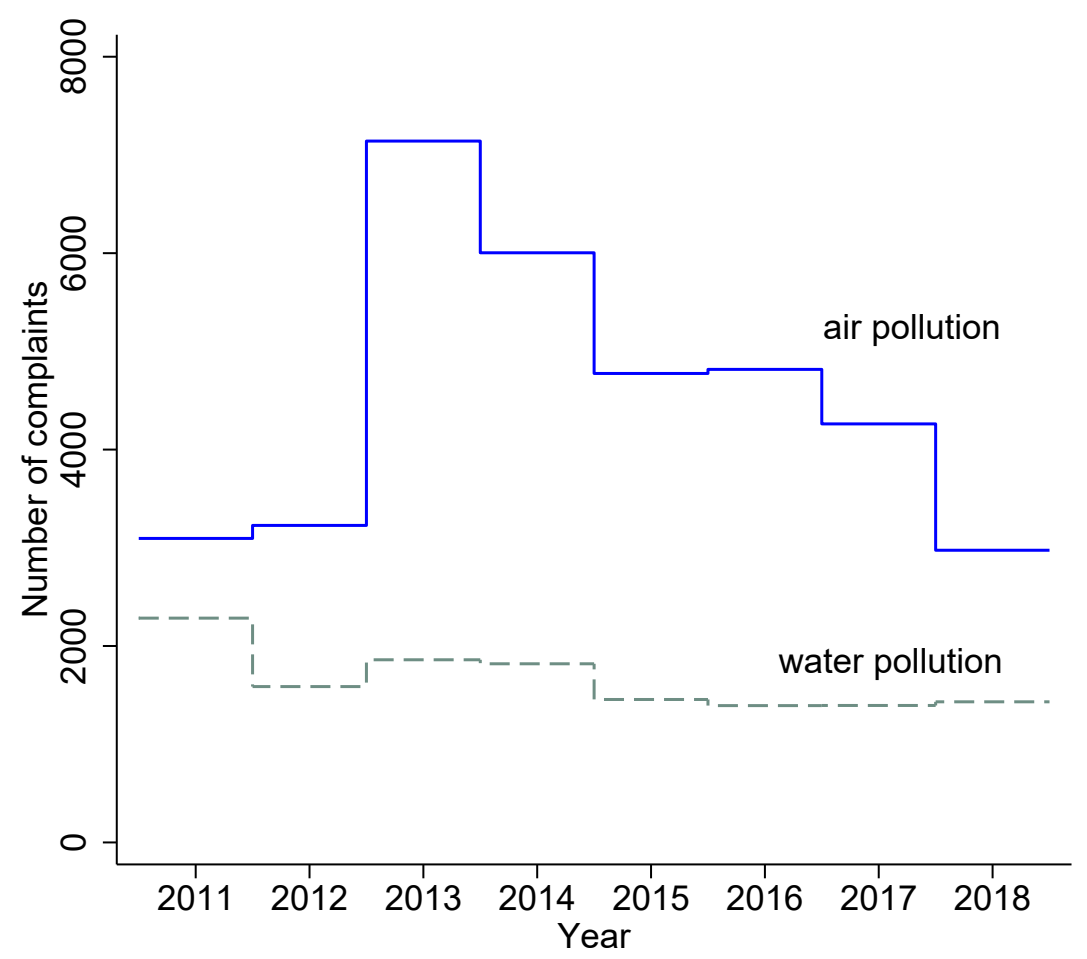

Notes: This figure plots the total number of complaints posted by citizens that are related to air pollution issues (solid line) and water pollution issues (dashed line) by year. Data are sourced from the message board for government leaders (Agarwal et al. 2020). 
Figure E.9: List of Cities by Rollout Waves

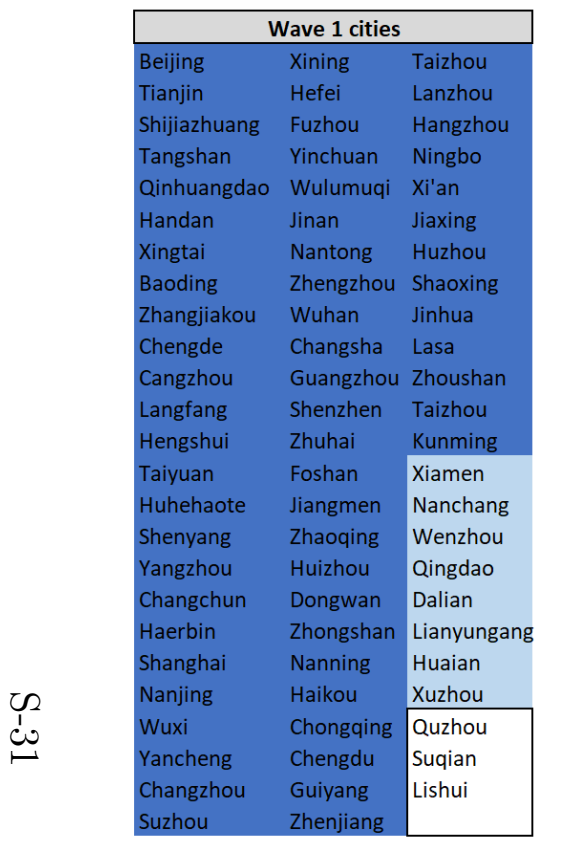

\begin{tabular}{llll|l|}
\hline \multicolumn{5}{|c|}{ Wave 2 cities } \\
\hline Wuhu & Jinzhou & Jimo & Wujiang & Yingkou \\
Maanshan & Zhuzhou & Pingdu & Changshu & Panjin \\
Datong & Xiangtan & Laixi & Zhangjiagang & Huludao \\
Yangquan & Yueyang & Zibo & Kunshan & Zigong \\
Changzhi & Changde & Zaozhuang & Taicang & Zhuji \\
Linfen & Zhangjiajie & Dongying & Haimen & Jiayuguan \\
Baotou & Shaoguan & Yantai & Jurong & Deyang \\
Chifeng & Shantou & Laizhou & Fuyang & Laiwu \\
Anshan & Zhanjiang & Penglai & Lin'an & Dezhou \\
Fushun & Pingdingshan & Zhaoyuan & Jiaozhou & Binzhou \\
Benxi & Anyang & Weifang & Yiwu & Heze \\
Yan'an & Jiaozuo & Shouguang & Jiujiang & Sanmenxia \\
Jingzhou & Jinchang & Jining & Quanzhou & Weinan \\
Yichang & Shizuishan & Taian & Eerduosi & Zhangqiu \\
Baoji & Kelamayi & Weihai & Wafangdian & Nanchong \\
Xianyang & Kuerle & Wendeng & Maoming & Yuxi \\
Jilin & Kaifeng & Rongcheng & Meizhou & \\
Qiqihaer & Luoyang & Rushan & Shanwei & \\
Daqing & Liuzhou & Rizhao & Heyuan & \\
Mudanjiang & Guilin & Zunyi & Yangjiang & \\
Jiaonan & Beihai & Linyi & Qingyuan & \\
Jiangyin & Sanya & Qujing & Chaozhou & \\
Yixing & Tongchuan & Liaocheng & Jieyang & \\
Liyang & Panzhihua & Mianyang & Yunfu & \\
Jintan & Luzhou & Yibin & Dandong & \\
\hline
\end{tabular}

\begin{tabular}{|c|c|c|c|c|c|c|c|}
\hline \multicolumn{8}{|c|}{ Wave 3 cities } \\
\hline & & Nanping & Ezhou & Guangyuan & Chuxiong & Dingxi & Shihezi \\
\hline Anqing & Hegang & Longyan & Jingmen & Suining & Honghe & Longnan & Wujiaqu \\
\hline Chuzhou & Shuangyashan & Ningde & Xiaogan & Neijiang & Wenshan & Linxia & \\
\hline Chizhou & Yichun & Jingdezhen & Huanggang & Leshan & Xishuangbanna & Gannan & \\
\hline Xuancheng & jiamusi & Pingxiang & Xianning & Meishan & Dali & Haidong & \\
\hline Lüliang & Qitaihe & Xinyu & Suizhou & Guangan & Dehong & Haibei & \\
\hline Wuhai & Heihe & Yingtan & Enshi & Dazhou & Nujiang & Huangnan & \\
\hline Tongliao & Suihua & Ganzhou & Hengyang & Yaan & Diqing & Hainan & \\
\hline Hulunbeier & Daxinganling & Ji'an & Shaoyang & Bazhong & Changdou & Guoluo & \\
\hline Bayannaoer & Bengbu & Yichun & Yiyang & Ziyang & Shannan & Yushu & \\
\hline Wulanchabu & Huainan & Fuzhou & Chenzhou & Aba & Rikaze & Haixi & \\
\hline Xingan & Huaibei & Shangrao & Yongzhou & Ganzi & Naqu & Wuzhong & \\
\hline Xilinguole & Jincheng & Hebi & Huaihua & Liangshan & Ali & Guyuan & \\
\hline Alashan & Shuozhou & Xinxiang & Loudi & Liupanshui & Linzhi & Zhongwei & \\
\hline Fuxin & Huangshan & Puyang & Xiangxi & Anshun & Hanzhong & Tulufan & \\
\hline Liaoyang & Jinzhong & Xuchang & Wuzhou & Bijie & Yulin & Hami & \\
\hline Tieling & Fuyang & Luohe & Fangchenggang & Tongren & Ankang & Changji & \\
\hline Chaoyang & Suzhou & Nanyang & Qinzhou & Qianxinan & Shangluo & Boertala & \\
\hline Siping & Liuan & Shangqiu & Guigang & Qiandongnan & Baiyin & Akesu & \\
\hline Liaoyuan & Haozhou & Xinyang & Yulin & Qiannan & Tianshui & Kezilesu & \\
\hline Tonghua & Yuncheng & Zhoukou & Baise & Baoshan & Wuwei & Kashi & \\
\hline Baishan & Xinzhou & Zhumadian & Hezhou & Shaotong & Zhangye & Hetian & \\
\hline Songyuan & Putian & Huangshi & Hechi & Lijiang & Pingliang & Yili & \\
\hline Baicheng & Sanming & Shiyan & Laibing & Puer & Jiuquan & Tacheng & \\
\hline Yanbian & Zhangzhou & Xiangyang & Chongzuo & Lincang & Qingyang & Aletai & \\
\hline
\end{tabular}

\section{Legend:}

Jing-Jin-Ji Metropolitan Region, Yangtze River Delta Economic Zone, Pearl River Delta Metropolitan Region, Direct-administered municipalities, Provincial Capitals Environmental Improvement Priority Cities (designated 2007), National Environmental Protection Exemplary Cities (awarded between 1997-2012)

Other prefecture-level cities

Notes: The three panels tabulate cities included in the official rollout waves of the monitoring program. Color coding indicates administrative hierarchies and predesignated status. Deep blues are cities in the Jing-Jin-Ji Metropolitan Region, the Yangtze River Delta Economic Zone, the Pearl River Delta Metropolitan Region, direct administered municipalities, and provincial capitals. Light blues are 2007 Environmental Improvement Priority Cities and 1997-2007 National Environmental Protection Exemplary Cities. White denotes remaining cities. 
Figure E.10: Pollution Avoidance Among Categories Most Sensitive to Precipitation

(a) $\%$ changes in transactions on days with precipitation by category

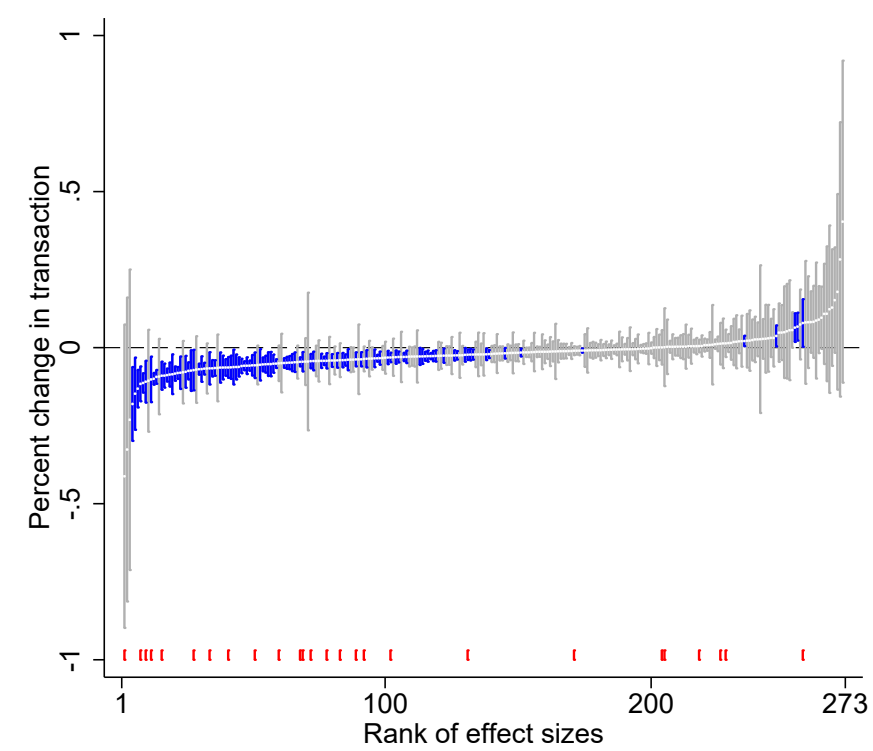

(b) Avoidance in most precipitation-sensitive categories

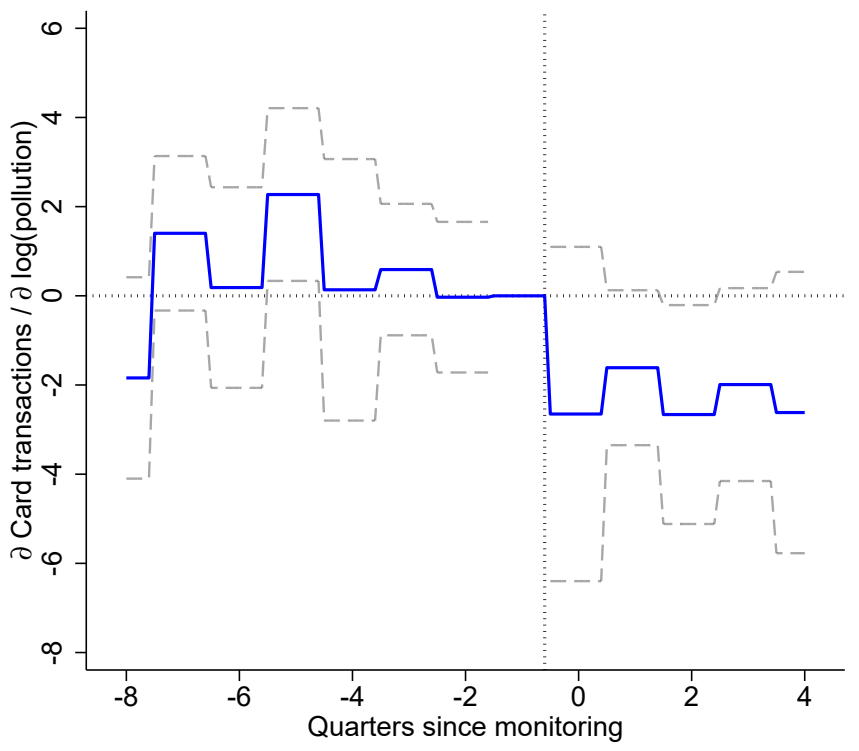

Notes: Panel (a) shows the impact of precipitation on spending for 273 merchant categories. To facilitate comparison across categories, for each category, we regress log city-day transactions on a dummy variable of rain or snow, controlling for city and week-of-year fixed effects. Each bar represents a separate merchant category. A bar's middle point denotes the percentage change in transactions on rainy/snowy days and its range corresponds to the $95 \%$ confidence interval. Blue (grey) color represents precise (imprecise) estimates. Red bars at the bottom mark merchant categories in supermarkets, dining, and entertainment sectors that are defined as deferrable in Table 2. Panel (b) conducts an event study using city-weekly transactions and limiting to the top 20 categories whose transactions are most sensitive to rain/snow. The underlying regression controls for city, week-of-year, and year fixed effects and city-specific time trends. Shaded region shows the $95 \%$ confidence interval constructed from standard errors clustered at the city level. 
Figure E.11: List of Top 20 Most Precipitation-Sensitive Merchant Categories

\begin{tabular}{|c|c|c|c|}
\hline MCC Code & MCC Name & Effect Size & $t$-stat \\
\hline 5598 & snow car dealers & -0.181 & -2.99 \\
\hline 7012 & timeshare vacation houses & -0.148 & -2.51 \\
\hline 7375 & information retrieval services & -0.131 & -4.30 \\
\hline 7941 & sports stadiums, clubs, playgrounds & -0.116 & -4.09 \\
\hline 5691 & garments store & -0.113 & -7.15 \\
\hline 7996 & amusement parks, circuses, carnivals & -0.109 & -3.20 \\
\hline 4733 & large resort ticket & -0.102 & -2.74 \\
\hline 8043 & opticians, optical products & -0.100 & -10.46 \\
\hline 5941 & sporting goods & -0.094 & -8.33 \\
\hline 7991 & tourism, exhibition & -0.090 & -3.93 \\
\hline 5661 & shoe store & -0.090 & -4.59 \\
\hline 5641 & children and baby goods & -0.088 & -8.30 \\
\hline 5271 & trailer dealer & -0.086 & -4.41 \\
\hline 5947 & gifts, souvenir & -0.085 & -2.63 \\
\hline 5977 & cosmetics store & -0.083 & -7.14 \\
\hline 7538 & auto service shops (non-dealer) & -0.081 & -8.41 \\
\hline 5621 & women's fashion store & -0.080 & -3.04 \\
\hline 5681 & fur store & -0.077 & -2.98 \\
\hline 5655 & sportswear & -0.074 & -5.33 \\
\hline 9399 & other government services & -0.074 & -2.22 \\
\hline 5300 & membership bulk retail & -0.072 & -2.18 \\
\hline
\end{tabular}

Notes: this table lists the top 20 most precipitation-sensitive merchant categories that are identified in panel (a) of Figure E.10. The event study in panel (b) of Figure E.10 uses these categories. "Effect Size" is the percentage changes in daily transaction volume in response to any precipitation, rain or snow, during the day. $t$-statistics are computed using standard errors clustered at the city level. 
Figure E.12: Visibility as Private Information of Air Pollution

(a) Distribution of daily visibility

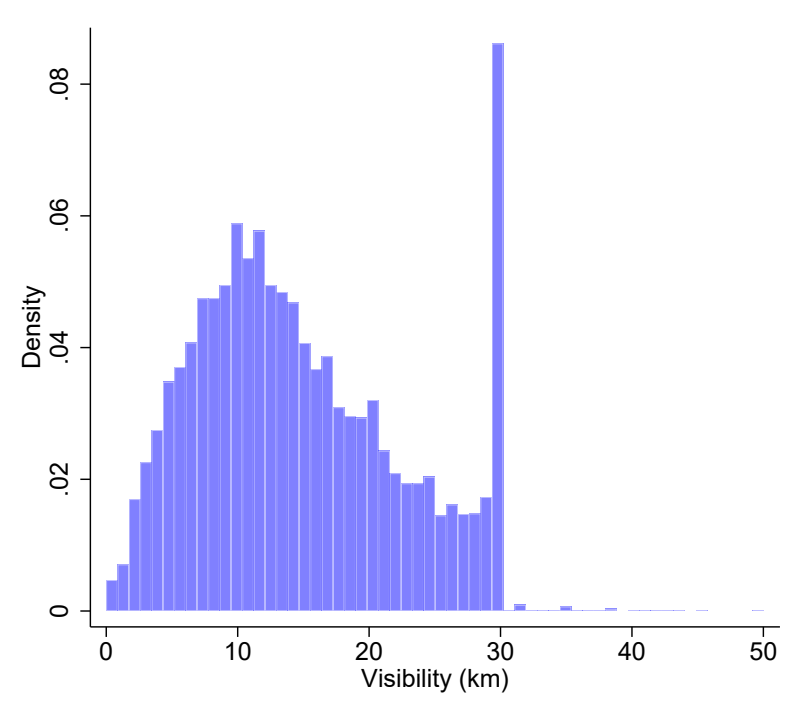

(c) Before monitoring

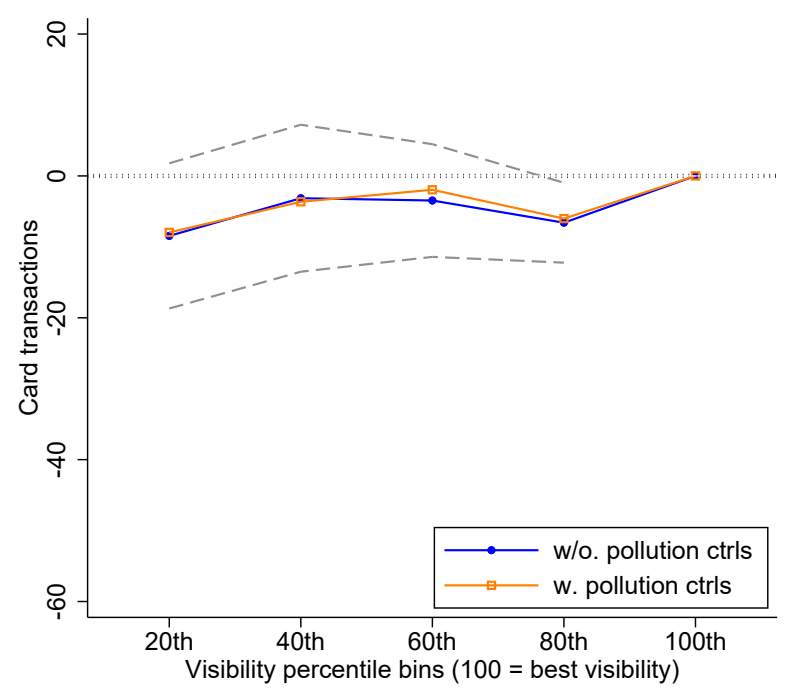

(b) Distribution of pollution by visibility

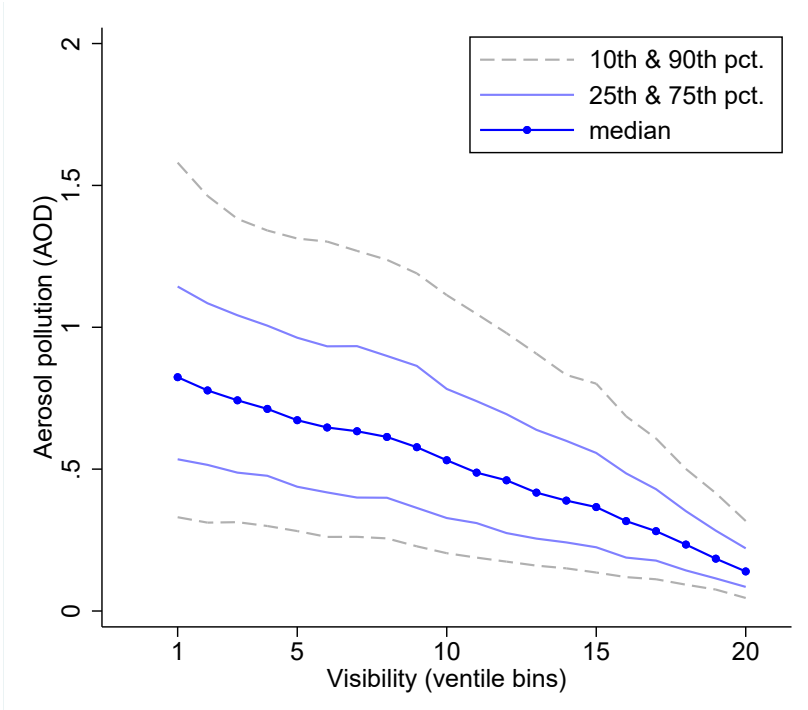

(d) After monitoring

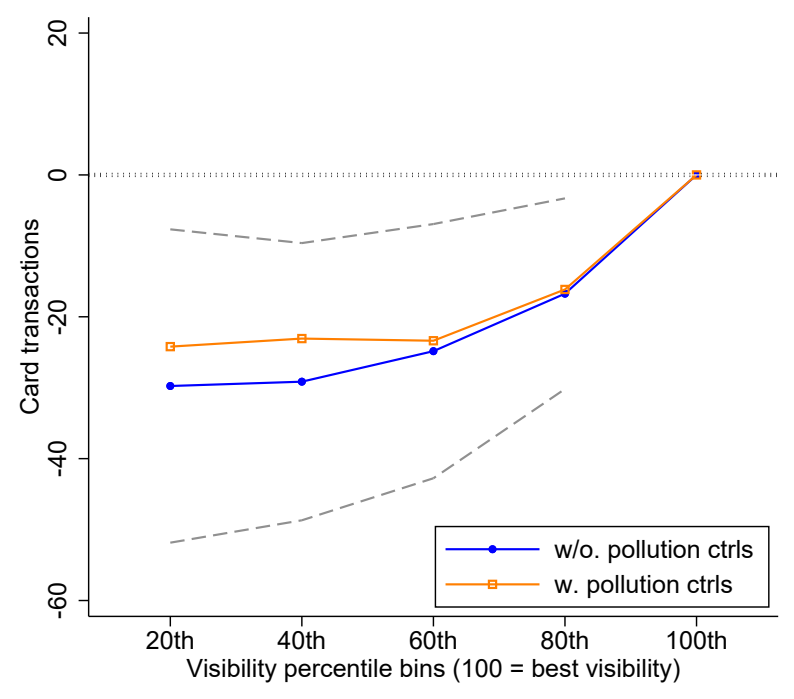

Notes: Panel (a) shows the distribution of raw visibility measure at the station-day level. For the vast majority of stations, visibility is top coded at $30 \mathrm{~km}$ (18.6 miles). Panel (b) shows the median, inter-quartile range, and 10-90 percentile range of satellite-based AOD by ventile bins of visibility. Panels (c) and (d) plot card transactions by visibility bins, separately for before and after the program. The underlying regressions control for city, week-of-year, and year fixed effects and city-specific linear time trends. The blue lines with solid circles show the effect of visibility without controlling for the pollution (AOD) levels. The orange lines show the effect of visibility controlling for pollution levels. Dashed lines show $95 \%$ confidence intervals for the no-pollution-controls specifications, calculated using standard errors clustered at the city level. 
Figure E.13: Awareness and Avoidance vs. U.S. Embassy $\mathrm{PM}_{2.5}$ Readings

(a) Residualized web searches

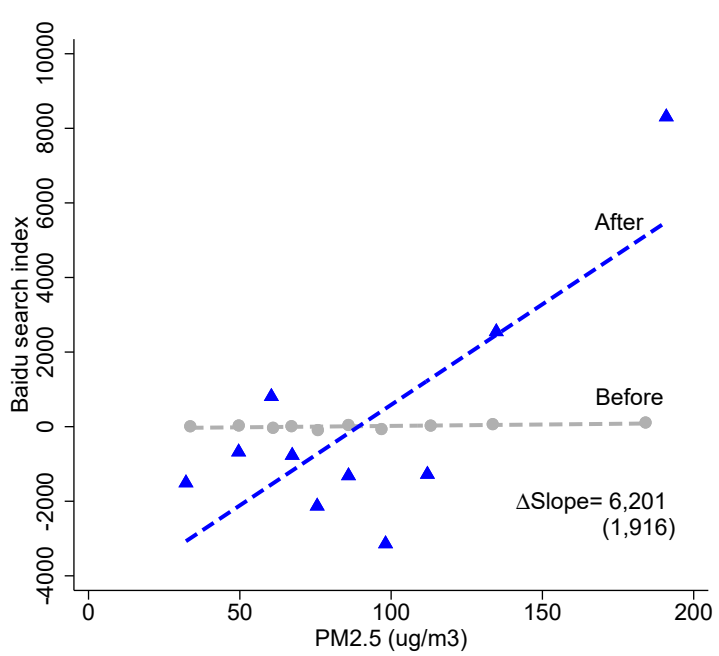

(b) Residualized card transactions

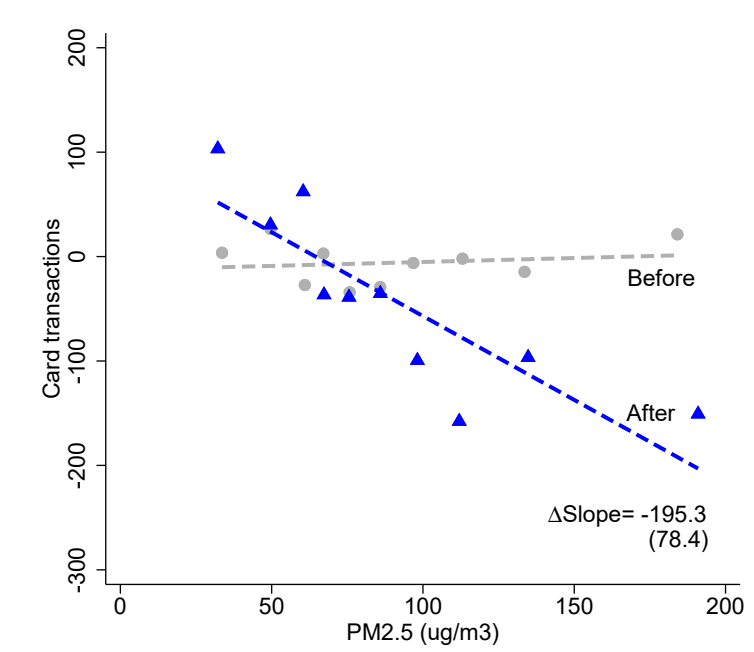

Notes: Each panel shows decile bin scatterplots of an outcome against U.S. Embassy's $\mathrm{PM}_{2.5}$ readings in Beijing, separately for before vs. after the monitoring program. Outcome variables are residualized Baidu searches for smog (panel a) and card transactions per 10,000 active cards (panel b) in Beijing after partialling out week-of-year fixed effects. " $\Delta$ Slope" reports the change in the outcome-pollution gradient after the monitoring policy. Robust standard errors are reported in parentheses. 
Figure E.14: Distribution of Cities by Rollout Dates

(a) Official rollout dates

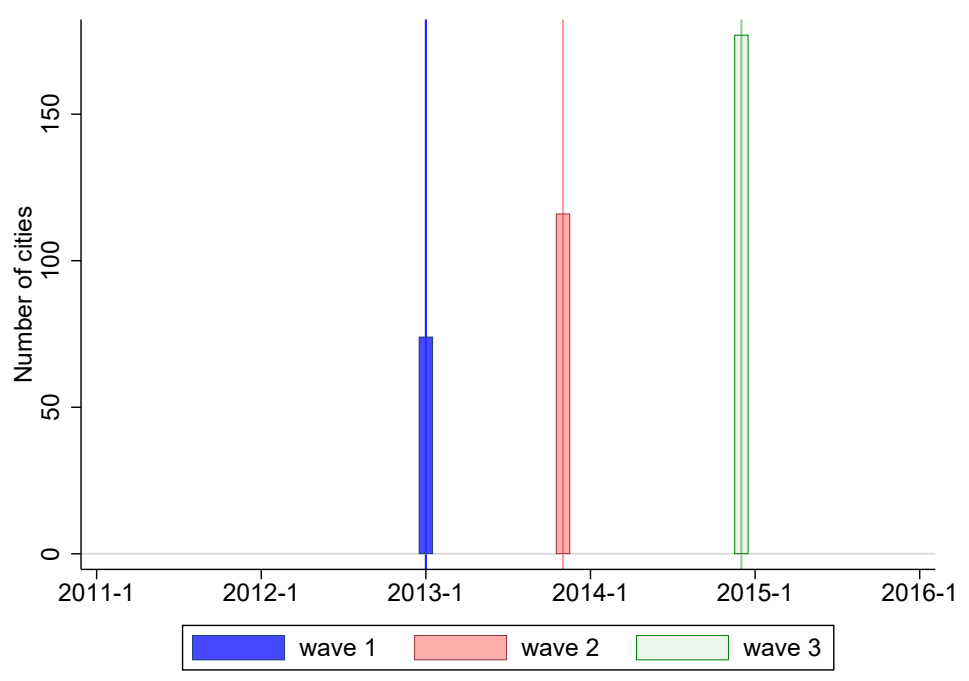

(b) "Field" rollout dates

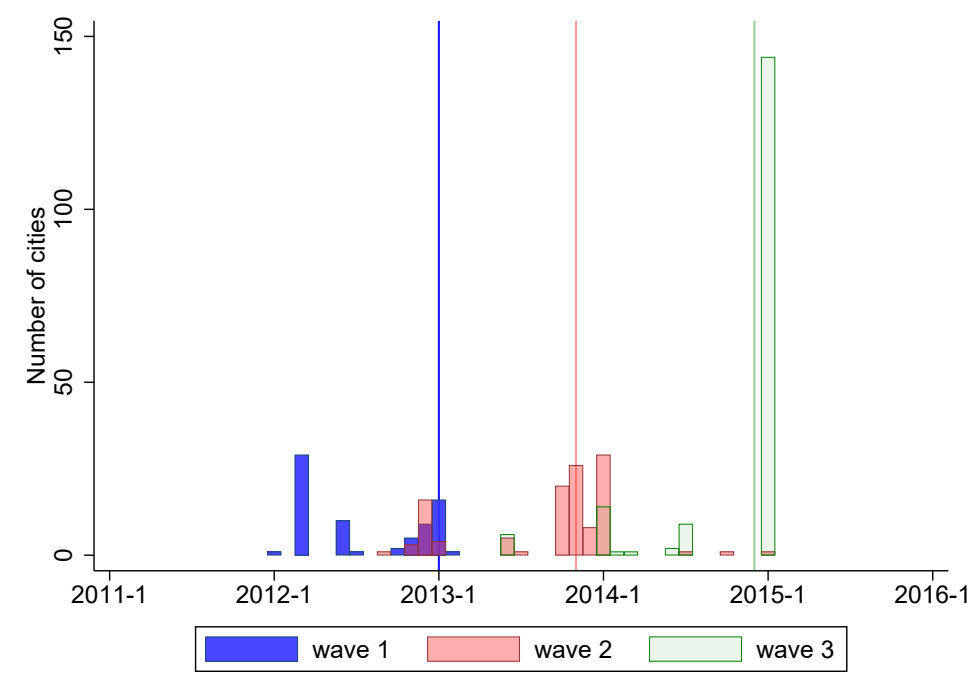

Notes: Panel (a) shows the number of cities for each official rollout dates. Panel (b) reports the number of cities for each "field" rollout date, the earliest date when a city's pollution monitoring data became available. We collected field rollout dates for over $92 \%$ of cities in our sample by searching news media and city government yearbooks. Overall, the field dates are close to the official dates. Using the field rollout dates produces similar results to those reported in the main text. 
Figure E.15: Staggered Rollout Design: Treated vs. Not-yet-Treated Comparison

(a) Illustration of comparison groups
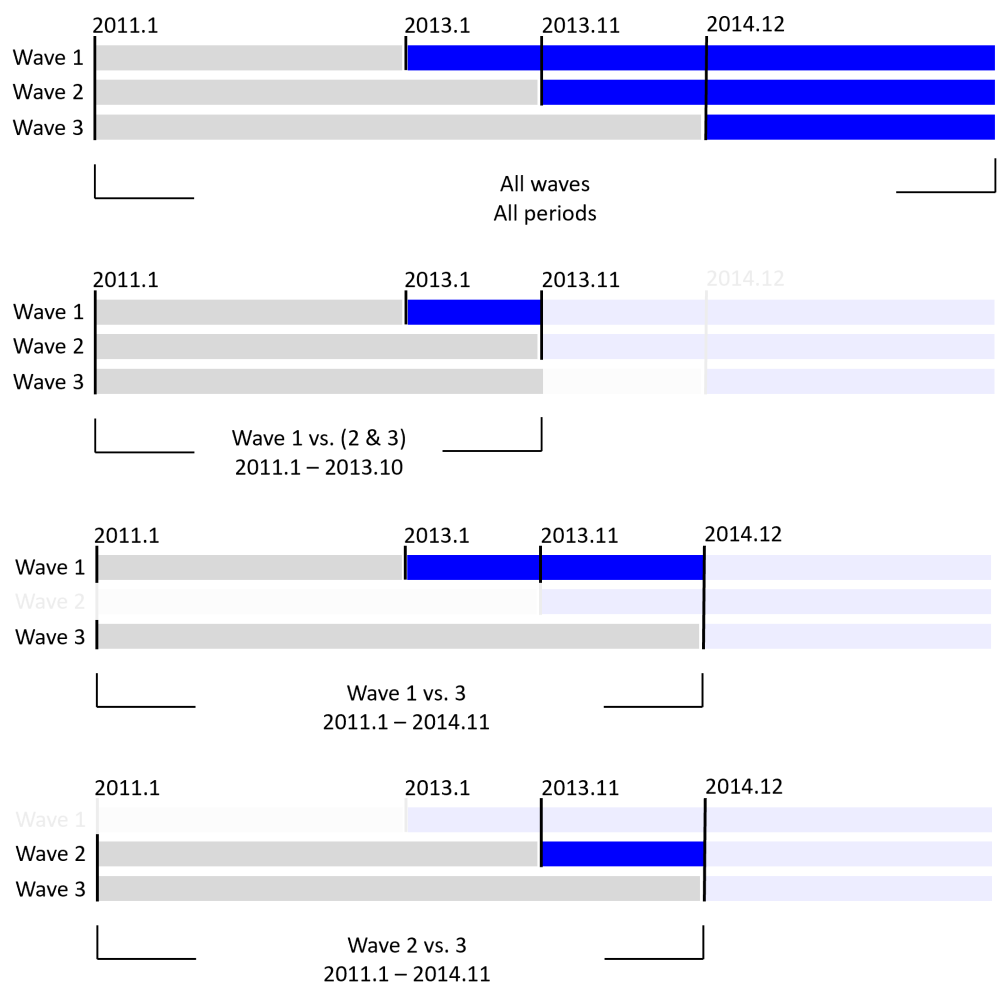

(b) Estimation results

\begin{tabular}{lcccc}
\hline & $\begin{array}{c}(1) \\
\text { All waves } \\
\text { All periods }\end{array}$ & $\begin{array}{c}\text { Wave 1vs(2\&3) } \\
\sim 2013.10\end{array}$ & $\begin{array}{c}(3) \\
\text { Wave 1vs3 } \\
\sim 2014.11\end{array}$ & $\begin{array}{c}(4) \\
\text { Wave 2vs3 } \\
\sim\end{array}$ \\
\cline { 2 - 5 } Panel A. Card transactions & & & & \\
\hline Log(Pollution) $\times 1$ (after monitoring) & -10.50 & -15.07 & -13.30 & -5.86 \\
& $(4.45)$ & $(6.49)$ & $(4.49)$ & $(3.96)$ \\
Observations & 83,122 & 44,224 & 45,433 & 47,623 \\
Panel B. Mortality & & & & \\
\hline Log(Pollution) $\times 1$ (after monitoring) & -0.023 & -0.026 & -0.030 & -0.053 \\
& $(0.007)$ & $(0.016)$ & $(0.013)$ & $(0.016)$ \\
Observations & 36,369 & 17,249 & 16,693 & 16,901 \\
\hline
\end{tabular}

Notes: Panel (a) illustrates the three comparison groups. Panel (b) reports estimation results using different comparison groups. Each panel-column is a separate regression using different cities and sample periods, as indicated by column head. "Log(Pollution)" is logged AOD in a city $\times$ week. In panel A, dependent variable is city $\times$ weekly bank card transactions per 10,000 active cards. Mean transaction rates are 869.1 (column 1), 821.1 (column 2), 850.1 (column 3), and 581.0 (column 4). In panel B, dependent variable is city $\times$ weekly $\log$ mortality rate. The coefficient of $\log ($ pollution $) \times 1$ (after monitoring) reports changes in mortality-pollution elasticity after monitoring. All regressions control for city, year, week-of-year fixed effects and city-specific time trends. Standard errors are clustered at the city level. 
Figure E.16: Upwind Cities Selected by the "Zero-Stage" Lasso Regression: Beijing

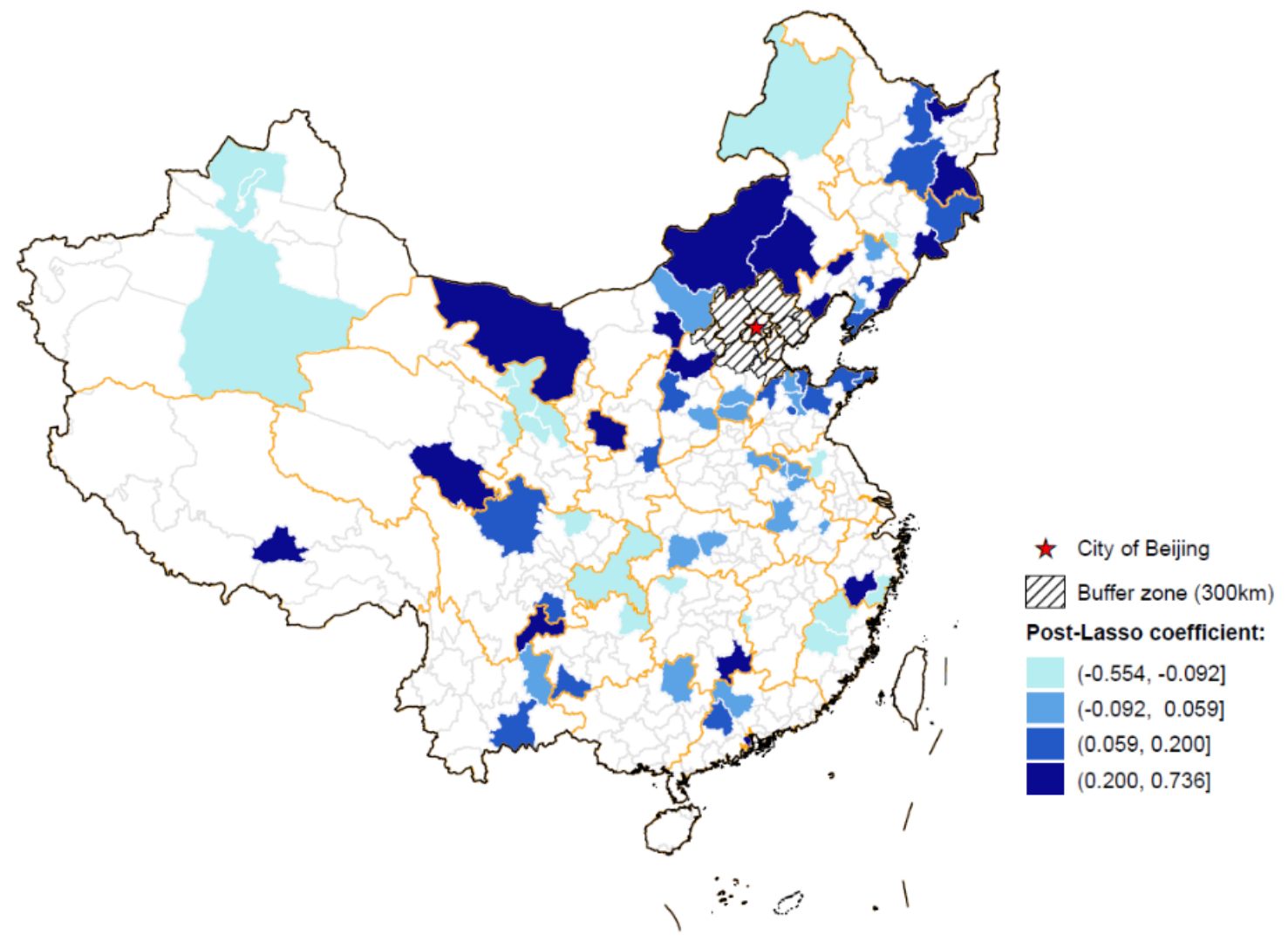

Notes: This map highlights 73 cities selected by a "zero-stage" Lasso regression of Beijing's daily AOD on all other 330 cities' upwind component vector AOD. 


\section{Figure E.17: OLS and IV Estimates of the Mortality-Pollution Gradient}

(a) OLS Estimates

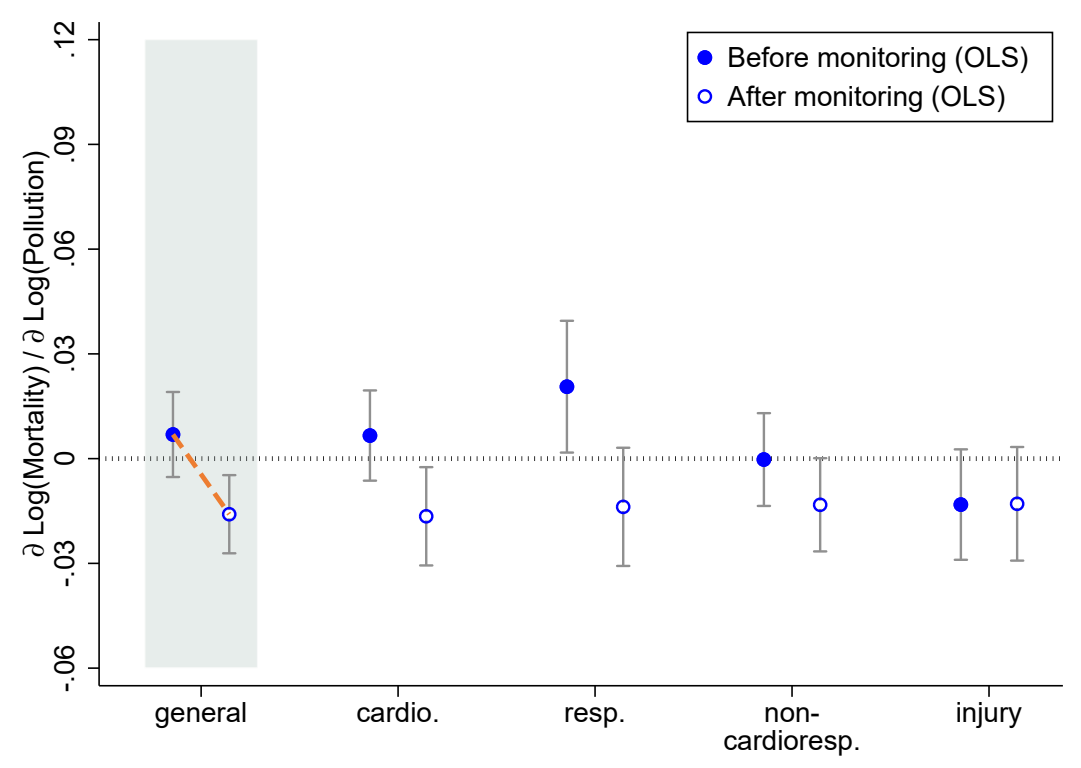

(b) IV Estimates

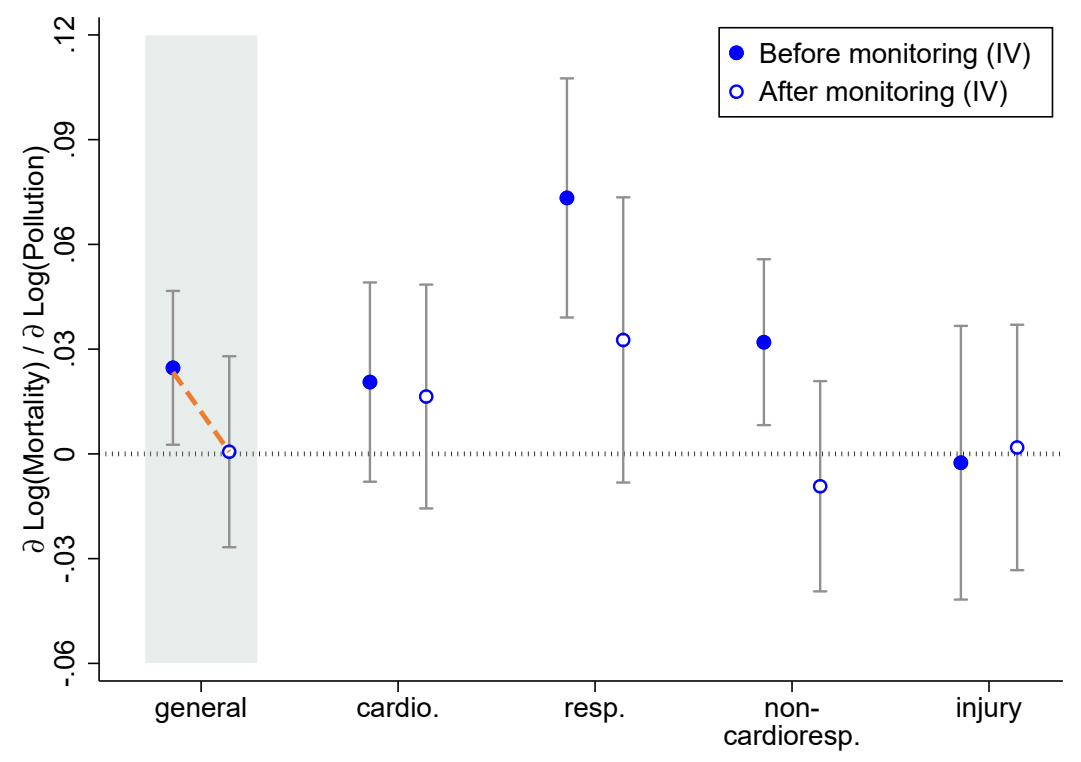

Notes: Panel (a) reports the OLS estimates of the mortality-pollution gradients by cause of death before the program (solid circles) and after the program (open circles). Panel (b) shows the IV estimates. We run separate regressions for each mortality cause. Both OLS and IV results suggest a reduction of the mortality-pollution gradient after the program. For all-cause mortality, the OLS and IV estimates exhibit virtually the same reduction in mortality-pollution gradient. The orange dashed lines, which have the same slope and length, serve as visual aid. This confirms the assumptions in Section 4.2: the magnitude of bias appears similar before vs. after monitoring and cancels out when we focus on the change in gradient. 
Figure E.18: The Monitoring Program's Mortality Impact - Synthetic Control Method

(a) Event study of the mortality impact

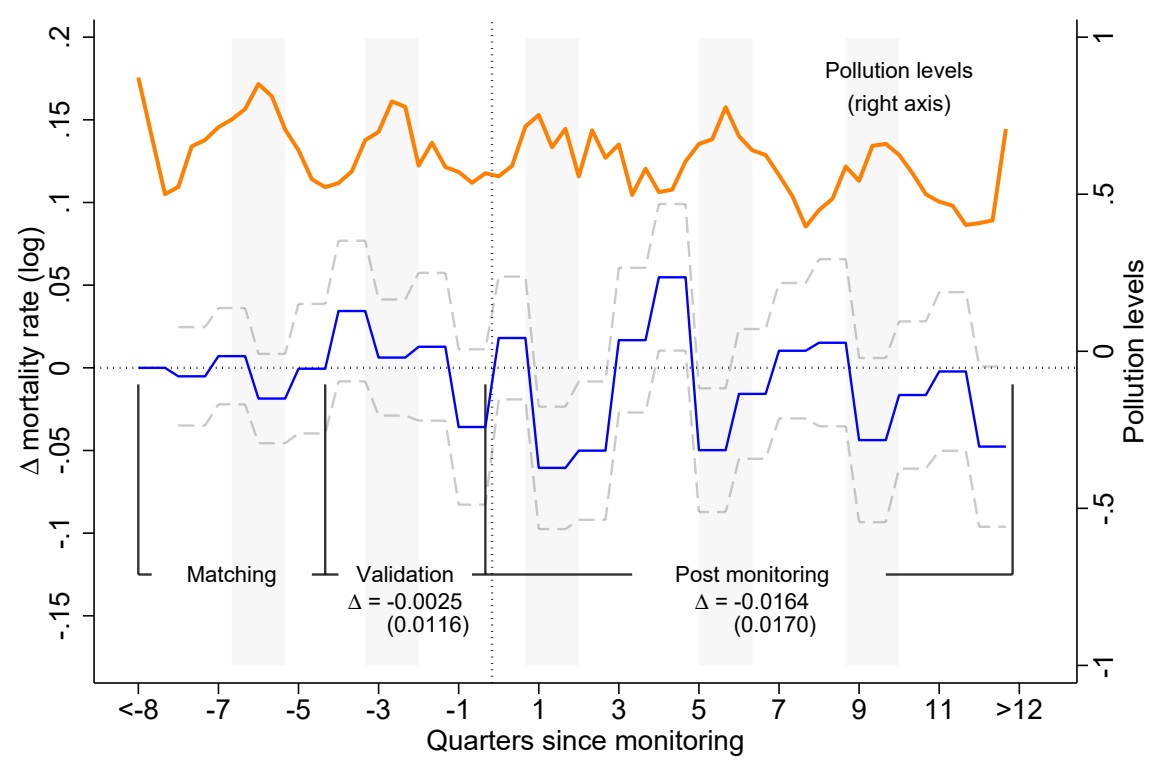

(b) Mortality impact by within-city pollution quintile

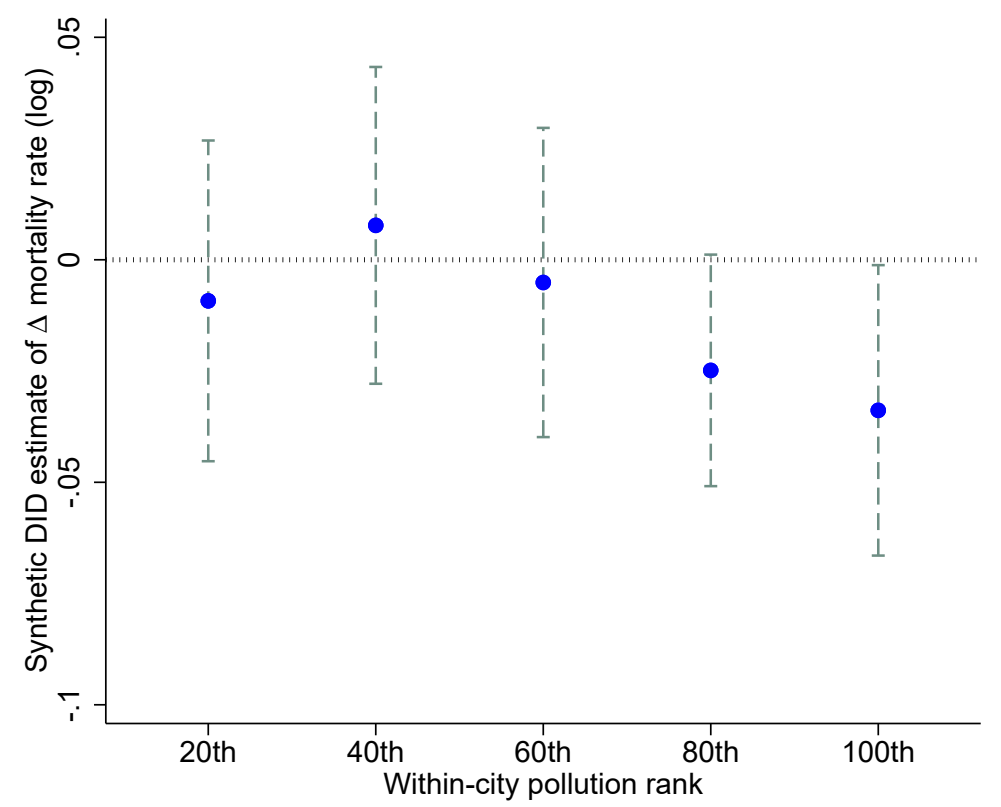

Notes: In panel (a), the blue line (and left axis) shows the difference between treated cities' mortality rates and their synthetically weighted control cities' quarterly mortality rates as a function of time relative to the information rollout, conditioning on city and quarter-of-year fixed effects. The orange line (and right axis) shows the treated cities' average monthly pollution level in the corresponding event time. Shaded areas highlight the quarters when a pollution peak occurred. Panel (b) shows the synthetic DID estimates of the monitoring program's mortality impact by within-city pollution quintiles. The dependent variable is city-week log mortality rate and the sample consists of city-weeks in different pollution quintiles.

Regressions control for city-by-group, week-of-year fixed, and year fixed effects, as well as group-specific linear time trends. Range bars display the $95 \%$ confidence intervals constructed using standard errors clustered at the city level. 
Table E.1: Chronology of Key Events Related to the Monitoring Program

\begin{tabular}{|c|c|}
\hline Time & Key Events \\
\hline Jun 2004 & $\begin{array}{l}\text { The first known use of the word "Smog" in news media is found in the weather } \\
\text { column of Beijing Daily, a popular local newspaper in Beijing }\end{array}$ \\
\hline Apr 2008 & $\begin{array}{l}\text { US Embassy in Beijing installs a rooftop air quality monitor and reports hourly } \\
\mathrm{PM}_{2.5} \text { via its twitter account @ @beijingair }\end{array}$ \\
\hline Apr 2009 & $\begin{array}{l}\text { A report by the National People's Congress recognizes } \mathrm{PM}_{2.5} \text { as a major pollutant, } \\
\text { a first in official public documents }\end{array}$ \\
\hline Jan 2010 & $\begin{array}{l}\text { China Meteorological Agency issues national standards on the observation and } \\
\text { forecast of smog based on visibility levels }\end{array}$ \\
\hline Nov 2010 & $\begin{array}{l}\text { First draft for NAAQS amendment opens for public comments, deeming the } \\
\text { choice of national } \mathrm{PM}_{2.5} \text { standards requires public input }\end{array}$ \\
\hline Nov 2011 & $\begin{array}{l}\text { Environmental NGOs start campaign "Measuring Air Quality for our Motherland" } \\
\text { and call for systematic } \mathrm{PM}_{2.5} \text { monitoring by the government }\end{array}$ \\
\hline Nov 2011 & $\begin{array}{l}\text { Second draft for NAAQS amendment opens for public comments, proposing } \\
\text { national standards for } \mathrm{PM}_{2.5} \text { for the first time }\end{array}$ \\
\hline Feb 2012 & NAAQS amended to set national standards for $\mathrm{PM}_{2.5}$ for the first time \\
\hline Jun 2012 & $\begin{array}{l}\text { Vice Minster of China's Ministry of Environmental Protection objects the release } \\
\text { of } \mathrm{PM}_{2.5} \text { data by US Embassy during a press conference }\end{array}$ \\
\hline Jan 2013 & $\begin{array}{l}\text { The first wave of Air Quality Monitoring and Disclosure program officially rolls out } \\
\text { in } 74 \text { cities, with two other waves to follow }\end{array}$ \\
\hline Sep 2013 & $\begin{array}{l}\text { China State Council issues "Air Pollution Prevention and Control Action Plan" } \\
\text { with } \mathrm{PM}_{2.5} \text {-reduction targets in key areas }\end{array}$ \\
\hline Mar 2014 & Chinese Premier Li Keqiang first declared "war on smog" \\
\hline
\end{tabular}

Notes: NAAQS stands for the National Ambient Air Quality Standards, first established in 1982. 
Table E.2: Changes in Web Searches-Pollution Gradient

\begin{tabular}{lcccc}
\hline Dep. var.: Standardized web searches for "smog" & & & \\
& $(1)$ & $(2)$ & $(3)$ & $(4)$ \\
\hline & 0.412 & 0.200 & 0.098 & 0.073 \\
$\log ($ Pollution) & $(0.286)$ & $(0.212)$ & $(0.105)$ & $(0.089)$ \\
& 0.205 & 0.339 & 0.438 & 0.371 \\
Log(Pollution) $\times 1$ (after monitoring) & $(0.105)$ & $(0.108)$ & $(0.154)$ & $(0.136)$ \\
& & & & \\
& $\checkmark$ & $\checkmark$ & $\checkmark$ & $\checkmark$ \\
FEs: city, city-linear time trends & $\checkmark$ & & & \\
FEs: month-of-year & $\checkmark$ & & & \\
FEs: year & & $\checkmark$ & $\checkmark$ & \\
FEs: month-of-sample & & & $\checkmark$ & $\checkmark$ \\
FEs: region $\times$ year & & & & $\checkmark$ \\
FEs: region $\times$ month-of-sample & & & & \\
\hline
\end{tabular}

Notes: Number of observations is 83,122 . "Log(Pollution)" is logged AOD in a city $\times$ week. "Region" is a conventional partition of cities by location: north (36 cities), northeast (38 cities), east (105 cities), central south (81 cities), southwest (54 cities), and northwest (52 cities). Standard errors are clustered at the city level. 
Table E.3: Determinants of Monitoring Roll-out Waves

\begin{tabular}{|c|c|c|c|c|c|c|}
\hline & $\begin{array}{c}(1) \\
1 \text { (wave-1) }\end{array}$ & $\begin{array}{c}(2) \\
1 \text { (wave-2) }\end{array}$ & $\begin{array}{c}(3) \\
1 \text { (wave-3) }\end{array}$ & $\begin{array}{c}(4) \\
\text { wave }\end{array}$ & $\begin{array}{c}(5) \\
\text { wave }\end{array}$ & $\begin{array}{c}(6) \\
\text { wave }\end{array}$ \\
\hline $1(\square)$ & $\begin{array}{c}0.925 \\
(0.032)\end{array}$ & $\begin{array}{c}0.015 \\
(0.015)\end{array}$ & $\begin{array}{c}0.060 \\
(0.029)\end{array}$ & $\begin{array}{l}-1.711 \\
(0.066)\end{array}$ & $\begin{array}{l}-1.467 \\
(0.108)\end{array}$ & \\
\hline $1(\square)$ & $\begin{array}{c}0.119 \\
(0.040)\end{array}$ & $\begin{array}{c}0.881 \\
(0.040)\end{array}$ & - & $\begin{array}{l}-0.964 \\
(0.049)\end{array}$ & $\begin{array}{l}-0.856 \\
(0.052)\end{array}$ & \\
\hline $1(\square)$ & $\begin{array}{c}0.015 \\
(0.009)\end{array}$ & $\begin{array}{c}0.125 \\
(0.023)\end{array}$ & $\begin{array}{c}0.860 \\
(0.025)\end{array}$ & - & - & \\
\hline Pollution level & & & & & $\begin{array}{l}-0.100 \\
(0.046)\end{array}$ & $\begin{array}{l}-0.254 \\
(0.079)\end{array}$ \\
\hline Pollution trends & & & & & $\begin{array}{c}-0.004 \\
(0.004)\end{array}$ & $\begin{array}{l}0.00022 \\
(0.0066)\end{array}$ \\
\hline Per capita income level & & & & & $\begin{array}{l}-0.654 \\
(0.231)\end{array}$ & $\begin{array}{l}-1.876 \\
(0.224)\end{array}$ \\
\hline Per capita income trends & & & & & $\begin{array}{c}0.143 \\
(0.156)\end{array}$ & $\begin{array}{l}-0.039 \\
(0.229)\end{array}$ \\
\hline Constant & & & & $\begin{array}{c}2.845 \\
(0.028)\end{array}$ & $\begin{array}{c}3.386 \\
(0.111)\end{array}$ & $\begin{array}{c}4.470 \\
(0.118)\end{array}$ \\
\hline $\mathrm{R}^{2}$ & 0.800 & 0.648 & 0.842 & 0.747 & 0.769 & 0.427 \\
\hline
\end{tabular}

Notes: In columns 1-3, the dependent variable 1(wave-k) is an indictor variable for whether a city is among the wave-k cities in the actual roll-out. In columns 4-6, the dependent variable is the roll-out wave as a "continuous" variable whose value equals 1, 2, or 3. The indicator variable 1(deep blue) denotes cities in the Jing-Jin-Ji Metropolitan Region, the Yangtze River Delta Economic Zone, the Pearl River Delta Metropolitan Region, direct administered municipalities, and provincial capitals. The indicator variable 1(light blue) denotes the 2007 designated Environmental Improvement Priority Cities and the 1997-2007 National Environmental Protection Exemplary Cities. The indicator variable 1(white) denotes the remaining cities. Variables 'Pollution level', 'Pollution trends', 'Per capita income level', and 'Per capita income trends' are divided by their mean (so the coefficients indicate percent changes). 
Table E.4: Characteristics of Cities by Monitoring Roll-out Waves

\begin{tabular}{|c|c|c|c|}
\hline & $(1)$ & $(2)$ & $(3)$ \\
\hline & Wave 1 & Wave 2 & Wave 3 \\
\hline Number of cities & 74 & 116 & 177 \\
\hline Population (million) & $\begin{array}{c}7.05 \\
(4.85)\end{array}$ & $\begin{array}{c}3.90 \\
(2.10)\end{array}$ & $\begin{array}{c}2.90 \\
(1.95)\end{array}$ \\
\hline GDP per capita (yuan) & $\begin{array}{c}69,836 \\
(27,627)\end{array}$ & $\begin{array}{c}42,881 \\
(23,110)\end{array}$ & $\begin{array}{c}27,400 \\
(13143)\end{array}$ \\
\hline AOD level & $\begin{array}{c}0.665 \\
(0.239)\end{array}$ & $\begin{array}{c}0.600 \\
(0.242)\end{array}$ & $\begin{array}{c}0.456 \\
(0.237)\end{array}$ \\
\hline $\mathrm{PM}_{2.5}$ level $\left(\mathrm{ug} / \mathrm{m}^{3}\right)$ & $\begin{array}{c}61.3 \\
(22.1)\end{array}$ & $\begin{array}{c}57.9 \\
(20.2)\end{array}$ & $\begin{array}{c}46.0 \\
(17.4)\end{array}$ \\
\hline Industrial $\mathrm{SO}_{2}$ emissions (ton) & $\begin{array}{c}37,569 \\
(40,186)\end{array}$ & $\begin{array}{c}29,609 \\
(24,695)\end{array}$ & $\begin{array}{c}18,214 \\
(17,550)\end{array}$ \\
\hline Average temperature (F) & $\begin{array}{c}59.7 \\
(8.52)\end{array}$ & $\begin{array}{c}58.0 \\
(9.59)\end{array}$ & $\begin{array}{c}55.3 \\
(10.6)\end{array}$ \\
\hline Total precipitation (inches) & $\begin{array}{c}47.0 \\
(21.9)\end{array}$ & $\begin{array}{c}42.2 \\
(23.2)\end{array}$ & $\begin{array}{c}40.3 \\
(24.4)\end{array}$ \\
\hline Average wind speed $(\mathrm{m} / \mathrm{s})$ & $\begin{array}{c}1.94 \\
(0.63)\end{array}$ & $\begin{array}{c}1.71 \\
(0.62)\end{array}$ & $\begin{array}{c}1.47 \\
(0.68)\end{array}$ \\
\hline
\end{tabular}

Notes: all characteristics are measured by the 2011-2015 average, except for $\mathrm{PM}_{2.5}$ (average over the post-monitoring periods) and industrial $\mathrm{SO}_{2}$ emissions (year 2006). The table reports the average characteristics of cities in different waves. Standard deviations are in parentheses. 
Table E.5: Changes in the Economic and Regulatory Environment After Monitoring

Indep. var.: 1(after monitoring)

$\begin{array}{llll}(1) & (2) & (3) & (4)\end{array}$

Panel A. Pollution levels

$\begin{array}{lcccc}\log (\text { Pollution }) & 0.0054 & 0.0044 & 0.0025 & -0.0042 \\ & (0.0095) & (0.0094) & (0.0096) & (0.0088) \\ \log (\max \text { Pollution }) & -0.0001 & -0.0077 & -0.0100 & -0.0140 \\ & (0.0127) & (0.0107) & (0.0109) & (0.0096)\end{array}$

Panel B. Political/regulatory environment

$\begin{array}{lcccc}{ }^{a} \mathrm{~N} \text { (anti-corruption cases) } & -0.004 & -0.028 & 0.010 & 0.005 \\ & (0.044) & (0.047) & (0.026) & (0.027) \\ { }^{\mathrm{b}} \text { Age(mayor) } & 0.335 & 0.313 & 0.350 & 0.354 \\ & (0.158) & (0.167) & (0.163) & (0.165) \\ { }^{\mathrm{c}} \text { Mayor having a Ph.D. degree } & -0.017 & -0.016 & -0.026 & -0.026 \\ & (0.023) & (0.025) & (0.025) & (0.025) \\ \mathrm{d} \mathrm{N} \text { ("pollution regulation" news mention) } & -0.0062 & -0.0092 & -0.0084 & -0.0084 \\ & (0.0064) & (0.0071) & (0.0074) & (0.0075)\end{array}$

Panel C. Healthcare access

${ }^{\mathrm{e}} \log \mathrm{N}($ hospitals per 100,000 people)

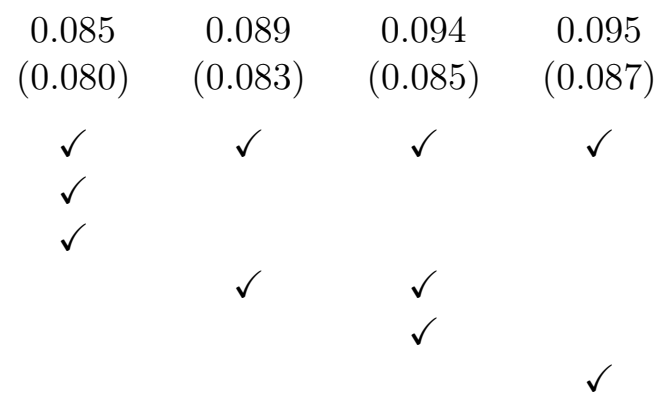

FEs: city, city-linear time trends

FEs: week-of-year

FEs: year

FEs: week-of-sample

FEs: region $\times$ year

FEs: region $\times$ week-of-sample

${ }^{\mathrm{a}} \mathrm{N}$ (anti-corruption cases)

$\begin{array}{ll}\text { mean }=0.24, & \mathrm{sd}=0.75 \\ \text { mean }=50.8, & \mathrm{sd}=3.63 \\ \text { mean }=0.234, & \mathrm{sd}=0.423 \\ \text { mean }=0.052, & \mathrm{sd}=0.45 \\ \text { mean }=4.56, & \mathrm{sd}=3.16\end{array}$

Notes: Each cell is a regression. Estimation data are at the city $\times$ weekly level, except for Panel $\mathrm{C}$ which uses city $\times$ annual hospital counts. Row names show the dependent variable. "Log(Pollution)" is logged AOD in a city $\times$ week. "Anti-corruption cases" is the number of local officials ousted during the anti-corruption campaign, "Mayor having a Ph.D. degree" indicates whether the current city mayor has a doctoral degree, "pollution regulation news" is the number of People's Daily news articles that mention both smog and the city name. "region" is a conventional partition of cities by location: north (36 cities), northeast (38 cities), east (105 cities), central south (81 cities), southwest (54 cities), and northwest (52 cities). Standard errors are clustered at the city level. *: $p<0.10 ; * *: p<0.05 ; * * *: p<0.01$. 
Table E.6: Changes in Air Purifier Purchase-Pollution Gradient

\begin{tabular}{lcccc}
\hline Dep. var.: Number of air purifier purchases per 1,000 people & \\
\hline & $(1)$ & $(2)$ & $(3)$ & $(4)$ \\
\hline & & & & \\
& 1.34 & -1.76 & -2.12 & -1.79 \\
$\log ($ Pollution) & $(1.33)$ & $(1.28)$ & $(1.25)$ & $(1.61)$ \\
& 1.86 & 6.21 & 6.69 & 6.50 \\
$\log ($ Pollution) $\times 1$ (after monitoring) & $(2.09)$ & $(2.44)$ & $(2.70)$ & $(3.26)$ \\
& & & & \\
& $\checkmark$ & $\checkmark$ & $\checkmark$ & $\checkmark$ \\
FEs: city, city-linear time trends & $\checkmark$ & & & \\
FEs: month-of-year & $\checkmark$ & & & \\
FEs: year & & $\checkmark$ & $\checkmark$ & \\
FEs: month-of-sample & & & $\checkmark$ & $\checkmark$ \\
FEs: region $\times$ year & & & & $\checkmark$ \\
FEs: region $\times$ month-of-sample & & & &
\end{tabular}

Notes: The analysis contains 50 cities for which we have air purifier sales data between 2012-2016. Number of observations is 9,871. Mean of dependent variable is 64.1 purchases per month per 1,000 city residents. "Log(Pollution)" is logged AOD in a city $\times$ month. These 50 cities account for $28 \%$ of the national population; among them, 34, 11, and 5 cities are in the first, second, and third wave of the information program rollout, respectively. All regressions include lower-order interaction and main effect terms. Standard errors are clustered at the city level. 
Table E.7: Changes in Bank Card Transaction-Pollution Gradient - Robustness

Coef. of interest: $\log ($ Pollution $) \times 1$ (after monitoring)

\begin{tabular}{lcccc} 
& $(1)$ & $(2)$ & $(3)$ & $(4)$ \\
\cline { 2 - 5 } Drop cities w/ top 10\% anti-corruption cases & -10.47 & -13.29 & -8.65 & -12.62 \\
& $(4.50)$ & $(5.26)$ & $(4.79)$ & $(5.53)$ \\
Control for online shopping shares & -10.58 & -13.21 & -7.86 & -12.02 \\
& $(4.54)$ & $(4.83)$ & $(4.41)$ & $(4.95)$ \\
Control for weather variables & -10.36 & -12.76 & -8.63 & -12.94 \\
Weekly max pollution & $(5.14)$ & $(5.54)$ & $(5.03)$ & $(5.68)$ \\
& -12.12 & -12.68 & -7.42 & -10.59 \\
Manually collected "field" roll-out date & $(5.97)$ & $(5.59)$ & $(4.37)$ & $(4.87)$ \\
Quarterly aggregation & -10.10 & -12.34 & -7.23 & -8.08 \\
& $(3.72)$ & $(3.85)$ & $(3.48)$ & $(3.75)$ \\
& -180.15 & -248.60 & -204.77 & -205.40 \\
& $(99.35)$ & $(149.89)$ & $(150.74)$ & $(135.80)$
\end{tabular}

FEs: city, city-linear time trends

FEs: week-of-year

FEs: year

FEs: week-of-sample

FEs: region $\times$ year

FEs: region $\times$ week-of-sample

Notes: This table examines the robustness of the changes in the transaction - pollution gradient. Each cell represents a separate regression. The main effect $\log ($ Pollution) term is not reported in the interest of space. Weather controls include linear terms of weekly temperature, precipitation, wind speed, barometric pressure, and their full interactions. Standard errors are clustered at the city level. Numbers of observations are (from top to bottom rows): 74903, 82703, 71857, 83122, 83122,6674 . 
Table E.8: Changes in Transaction-Pollution Gradient - Spatial Spillovers

Dep. var.: Number of transactions per 10,000 active cards in a city $\times$ week

\begin{tabular}{lcccc} 
& & & & \\
& $(1)$ & $(2)$ & $(3)$ & $(4)$ \\
\cline { 2 - 5 } $\log ($ Pollution) & & & & \\
& 0.15 & -0.29 & -0.36 & -0.28 \\
& $(0.71)$ & $(0.83)$ & $(0.54)$ & $(0.63)$ \\
Log(Pollution) $\times 1$ (after monitoring) $\times 1$ (Treated) & -9.05 & -10.03 & -9.00 & -10.66 \\
& $(5.71)$ & $(7.34)$ & $(6.85)$ & $(8.00)$ \\
& & & & \\
FEs: city-group, city-linear time trends & $\checkmark$ & $\checkmark$ & $\checkmark$ & $\checkmark$ \\
FEs: week-of-year & $\checkmark$ & & & \\
FEs: year & $\checkmark$ & & & \\
FEs: week-of-sample & & $\checkmark$ & $\checkmark$ & \\
FEs: region $\times$ year & & & $\checkmark$ & \\
FEs: region $\times$ week-of-sample & & & & $\checkmark$ \\
\hline
\end{tabular}

Notes: Number of observations is 150,289. A treated city and its neighboring cities not yet experiencing the roll-out constitute a city-group. "Log(Pollution)" is logged AOD in a city $\times$ week. "1(Treated)" equals 1 for cities in the roll-out wave and 0 for neighboring cities not yet experiencing the roll-out. "region" is a conventional partition of cities by location: north (36 cities), northeast (38 cities), east (105 cities), central south ( 81 cities), southwest (54 cities), and northwest (52 cities). All regressions include lower-order interaction and main effect terms. All fixed effects are interacted with 1 (Treated) dummy. Standard errors are clustered at the city level. *: $p<0.10$ **: $^{*}<0.05$ *** $^{* *}<0.01$. 
Table E.9: Changes in Card Transaction-Pollution Gradient: Visibility

\begin{tabular}{|c|c|c|c|c|c|c|c|c|}
\hline & $(1)$ & $(2)$ & $(3)$ & $(4)$ & $(5)$ & $(6)$ & $(7)$ & $(8)$ \\
\hline $\log (1 /$ Visibility $)$ & $\begin{array}{l}10.12 \\
(6.59)\end{array}$ & $\begin{array}{c}3.08 \\
(6.18)\end{array}$ & $\begin{array}{c}3.96 \\
(5.96)\end{array}$ & $\begin{array}{l}15.46 \\
(7.01)\end{array}$ & $\begin{array}{c}11.46 \\
(10.57)\end{array}$ & $\begin{array}{c}0.23 \\
(9.57)\end{array}$ & $\begin{array}{c}1.51 \\
(7.86)\end{array}$ & $\begin{array}{l}10.20 \\
(8.88)\end{array}$ \\
\hline $\log (1 /$ Visibility $) \times 1($ after monitoring $)$ & $\begin{array}{l}-11.98 \\
(12.92)\end{array}$ & $\begin{array}{l}-13.49 \\
(13.28)\end{array}$ & $\begin{array}{l}-7.54 \\
(9.92)\end{array}$ & $\begin{array}{l}-10.86 \\
(10.64)\end{array}$ & $\begin{array}{c}-6.05 \\
(16.40)\end{array}$ & $\begin{array}{c}-1.52 \\
(18.72)\end{array}$ & $\begin{array}{c}2.95 \\
(13.54)\end{array}$ & $\begin{array}{c}1.60 \\
(14.71)\end{array}$ \\
\hline Log(Pollution) & & & & & $\begin{array}{c}3.25 \\
(5.19)\end{array}$ & $\begin{array}{c}3.47 \\
(5.63)\end{array}$ & $\begin{array}{c}2.09 \\
(4.20)\end{array}$ & $\begin{array}{c}3.21 \\
(4.64)\end{array}$ \\
\hline $\log ($ Pollution $) \times 1$ (after monitoring $)$ & & & & & $\begin{array}{l}-9.38 \\
(4.86)\end{array}$ & $\begin{array}{l}-12.88 \\
(8.01)\end{array}$ & $\begin{array}{l}-9.06 \\
(6.42)\end{array}$ & $\begin{array}{l}-12.87 \\
(7.54)\end{array}$ \\
\hline FEs: city, city-linear time trends & $\checkmark$ & $\checkmark$ & $\checkmark$ & $\checkmark$ & $\checkmark$ & $\checkmark$ & $\checkmark$ & $\checkmark$ \\
\hline FEs: week-of-year & $\checkmark$ & & & & $\checkmark$ & & & \\
\hline FEs: year & $\checkmark$ & & & & $\checkmark$ & & & \\
\hline FEs: week-of-sample & & $\checkmark$ & $\checkmark$ & & & $\checkmark$ & $\checkmark$ & \\
\hline FEs: region $\times$ year & & & $\checkmark$ & & & & $\checkmark$ & \\
\hline FEs: region $\times$ week-of-sample & & & & $\checkmark$ & & & & $\checkmark$ \\
\hline
\end{tabular}

Notes: Number of observations is $83,122$. "Log(1/Visibility)" is logged inverse visibility in a city $\times$ week that is based on weather-station data sourced from China's National Meteorological Administration. "Log(Pollution)" is logged AOD in a city $\times$ week. Dependent variable is city $\times$ weekly bank card transactions per 10,000 active cards. "region" is a conventional partition of cities by location: north (36 cities), northeast (38 cities), east (105 cities), central south (81 cities), southwest (54 cities), and northwest (52 cities). Standard errors are clustered at the city level. 
Table E.10: Annual Analysis using Alternative Pollution Measurements

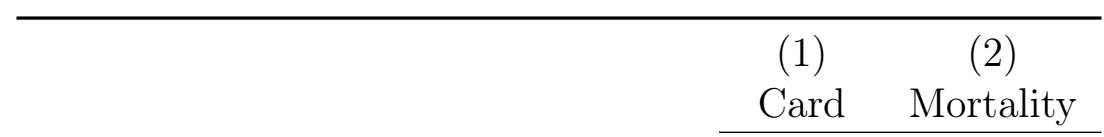

Panel A. Pollution = Modeling $\mathrm{PM}_{2.5}$ near monitoring site

$\begin{array}{lcc}\log (\text { Pollution }) & -656.6 & 0.039 \\ & (349.2) & (0.049) \\ \log (\text { Pollution }) \times 1(\text { after monitoring) } & -167.7 & -0.032 \\ & (173.3) & (0.087)\end{array}$

Panel B. Pollution $=$ Modeling $\mathrm{PM}_{2.5}$ citywide

\begin{tabular}{lcc}
\hline Log(Pollution) & -490.3 & 0.032 \\
& $(280.2)$ & $(0.049)$ \\
$\log ($ Pollution $) \times 1$ (after monitoring) & -282.9 & -0.054 \\
& $(119.4)$ & $(0.092)$ \\
& & \\
Panel C. Pollution = AOD & & \\
\hline & & \\
Log(Pollution) & 343.6 & 0.119 \\
& $(181.3)$ & $(0.058)$ \\
Log(Pollution) $\times 1($ after monitoring) & -329.6 & -0.111 \\
& $(147.5)$ & $(0.076)$ \\
& & \\
Observations & 1,998 & 774 \\
FEs: city & $\checkmark$ & $\checkmark$ \\
FEs: year & $\checkmark$ & $\checkmark$ \\
\hline
\end{tabular}

Notes: $\log \left(\right.$ Pollution) in Panels A and B is based on the modeling $\mathrm{PM}_{2.5}$ measure from Van Donkelaar et al. (2016) at the 10km-grid-by-annual frequency. For each city, Panel A averages over the annual pollution readings from grids that are closest to the city's monitoring stations, while Panel B averages over all grids within the city. $\log ($ Pollution) in Panel C is the city-annual AOD measure. Dependent variables are the number of annual transactions per 10,000 active cards in each city (column 1 ) and the $\log$ mortality rate in the city year (column 2). Standard errors are clustered at the city level. 
Table E.11: Heterogeneity by City Characteristics

\begin{tabular}{|c|c|c|c|c|c|}
\hline City characteristics: & $\begin{array}{c}\text { (1) } \\
\text { Per cap. } \\
\text { income }\end{array}$ & $\begin{array}{c}(2) \\
\text { Frac. } \\
\text { urban }\end{array}$ & $\begin{array}{c}\text { (3) } \\
\text { Per cap. } \\
\text { hospitals }\end{array}$ & $\begin{array}{l}\quad(4) \\
\text { Per cap. } \\
\text { residential } \\
\text { electricity }\end{array}$ & $\begin{array}{c}(5) \\
\text { Per cap. } \\
\text { mobile } \\
\text { phones }\end{array}$ \\
\hline \multicolumn{6}{|l|}{ Panel A. Card transactions } \\
\hline $\begin{aligned} \log (\text { Pollution }) & \times 1(\text { after monitoring }) \\
& \times 1 \text { (below average })\end{aligned}$ & $\begin{array}{l}-4.99 \\
(3.34)\end{array}$ & $\begin{array}{l}-7.26 \\
(4.82)\end{array}$ & $\begin{array}{l}-6.40 \\
(5.57)\end{array}$ & $\begin{array}{l}-7.84 \\
(3.02)\end{array}$ & $\begin{array}{l}-11.00 \\
(3.30)\end{array}$ \\
\hline $\begin{aligned} \log (\text { Pollution }) & \times 1(\text { after monitoring }) \\
& \times 1(\text { above average })\end{aligned}$ & $\begin{array}{l}-9.22 \\
(6.79)\end{array}$ & $\begin{array}{l}-10.80 \\
(7.35)\end{array}$ & $\begin{array}{l}-13.17 \\
(7.70)\end{array}$ & $\begin{array}{l}-10.14 \\
(7.04)\end{array}$ & $\begin{array}{l}-11.81 \\
(6.84)\end{array}$ \\
\hline \multicolumn{6}{|l|}{ Panel B. Mortality } \\
\hline $\begin{aligned} \log (\text { Pollution }) & \times 1(\text { after monitoring }) \\
& \times 1 \text { (below average })\end{aligned}$ & $\begin{array}{l}-0.019 \\
(0.008)\end{array}$ & $\begin{array}{l}-0.018 \\
(0.009)\end{array}$ & $\begin{array}{l}-0.014 \\
(0.008)\end{array}$ & $\begin{array}{l}-0.020 \\
(0.010)\end{array}$ & $\begin{array}{l}-0.014 \\
(0.009)\end{array}$ \\
\hline $\begin{aligned} \log (\text { Pollution }) & \times 1(\text { after monitoring }) \\
& \times 1(\text { above average })\end{aligned}$ & $\begin{array}{l}-0.035 \\
(0.011)\end{array}$ & $\begin{array}{l}-0.033 \\
(0.008)\end{array}$ & $\begin{array}{l}-0.038 \\
(0.013)\end{array}$ & $\begin{array}{l}-0.046 \\
(0.011)\end{array}$ & $\begin{array}{l}-0.041 \\
(0.010)\end{array}$ \\
\hline
\end{tabular}

Notes: This table reports heterogeneous changes in the purchase-pollution gradient (panel A) and mortality-pollution gradient (panel B) by above and below average city characteristics. Each column corresponds to a separate city characteristic: column 1 = per capita personal dispensable income; column $2=$ share of urban population; column $3=$ per capita number of hospitals; column $4=$ per capita residential electricity usage; column $5=$ share of mobile phone users. City characteristics are computed as the 2011-2015 average. Cities with missing attributes are omitted from the analysis. All regressions control for city, week-of-year, and year fixed effects, full sets of lower-order interaction terms, and city-specific time trends. Standard errors are clustered at the city level. 